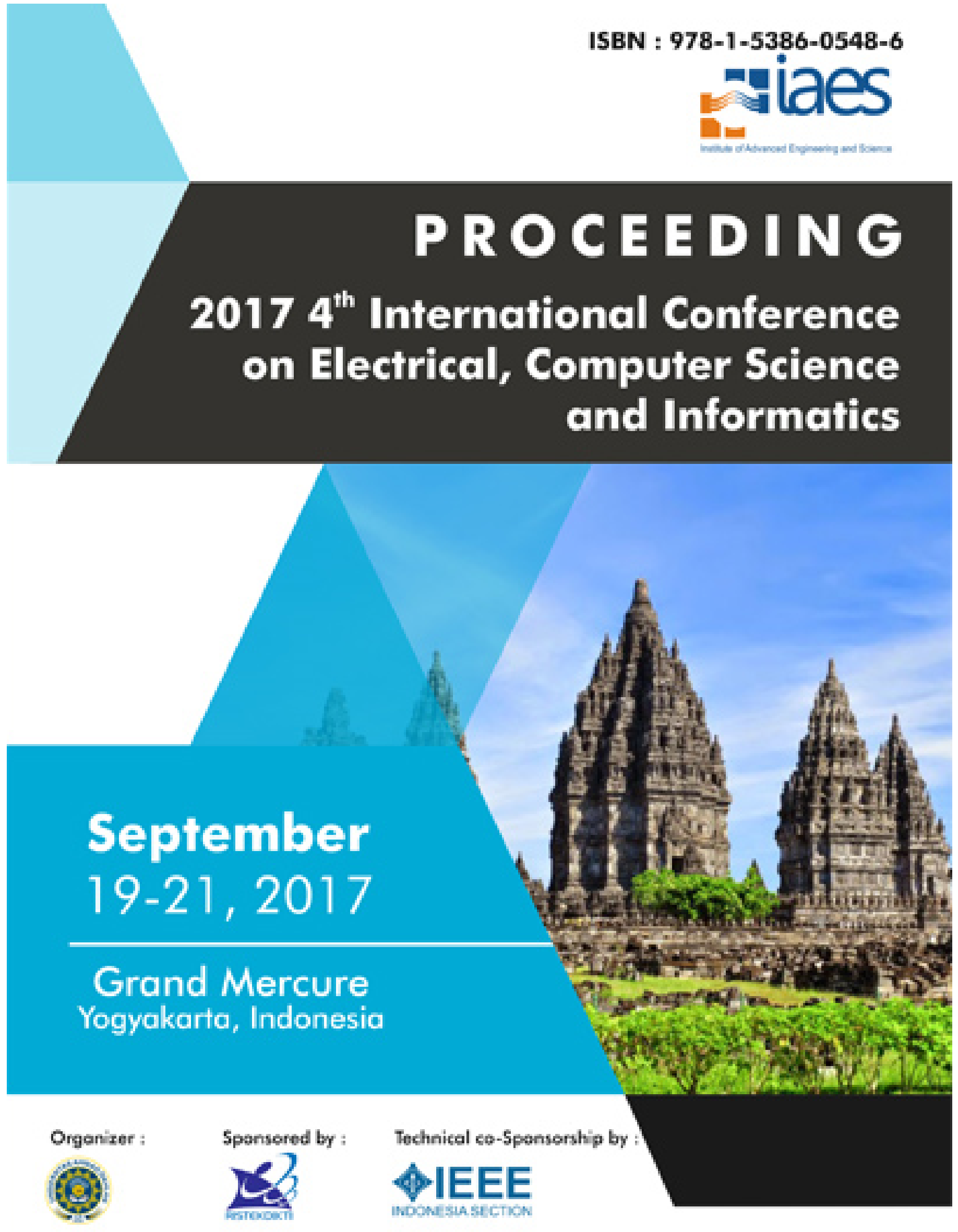

co-Orgonisor:

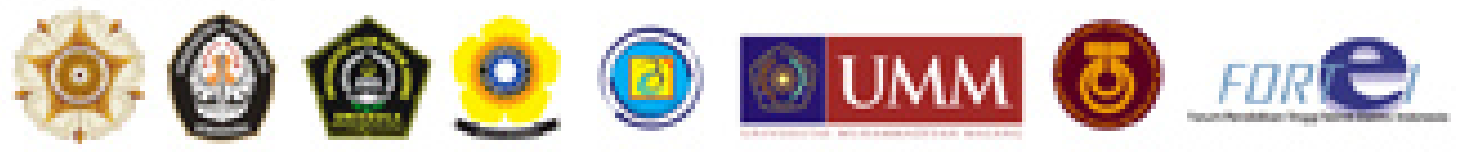




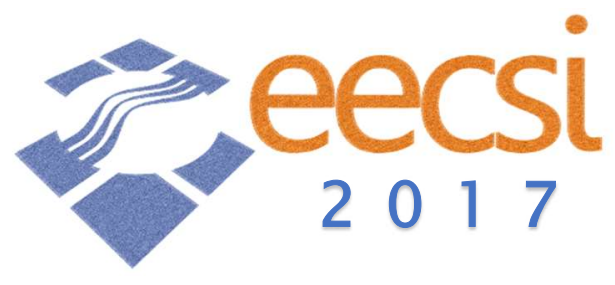

\title{
PROCEEDINGS
}

\section{4th International Conference on Electrical Engineering, Computer Science and Informatics (EECSI)}

19-21 September 2017, Yogyakarta, Indonesia

\author{
Editors: \\ Munawar A Riyadi \\ Mochammad Facta \\ Deris Stiawan \\ Hatib Rahmawan
}




\section{Proceedings}

\section{4th International Conference on Electrical Engineering, Computer Science and Informatics (EECSI)}

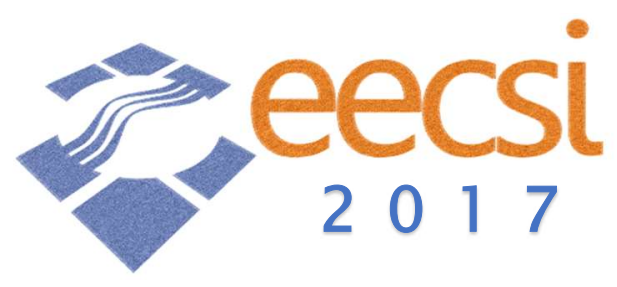

Copyright and Reprint Permission: Abstracting is permitted with credit to the source. Libraries are permitted to photocopy beyond the limit of U.S. copyright law for private use of patrons those articles in this volume that carry a code at the bottom of the first page, provided the per-copy fee indicated in the code is paid through Copyright Clearance Center, 222 Rosewood Drive, Danvers, MA 01923. For other copying, reprint or republication permission, write to IEEE Copyrights Manager, IEEE Operations Center, 445 Hoes Lane, Piscataway, NJ 08854. All rights reserved.

Copyright (C) 2017 by IEEE.

ISBN : 978-1-5386-0548-6 (PRINT, Part Number : CFP17B51-PRT)

ISBN : 978-1-5386-0547-9 (DVD Part Number : CFP17B51-CDR)

ISBN : 978-1-5386-0549-3 (XPLORE COMPLIANT, Part Number : CFP17B51-ART)

Additional copies may be ordered to:

Lembaga Penerbitan dan Publikasi Ilmiah (LPPI)

Universitas Ahmad Dahlan

Jl. Kapas 9, Semaki, Umbulharjo

Yogyakarta, Indonesia 55166 


\section{ORGANIZING COMMITTEE OF EECSI 2017 CONFERENCE}

\section{Steering Committee}

- Adam Skorek, IEEE MGA Awards and Recognation Chair (R7) Trois-Rivières, QC, Canada

- Satrio Dharmanto, IEEE Indonesia Section (Chair)

- Pekik Argo Dahono, IEEE Indonesia Chapters Chair (EdSoc/EDS/PELS/SPS)

- Mochamad Ashari, Telkom University, Bandung, Indonesia

- Tumiran, Universitas Gadjah Mada, Yogyakarta, Indonesia

- Hermawan, Universitas Diponegoro, Semarang, Indonesia

- Zainudin Nawawi, Universitas Sriwijaya, Palembang, Indonesia

- Rahmat Budiarto, Albaha University, Baha, Saudi Arabia

- Sri Arttini Dwi Prasetyowati, Universitas Islam Sultan Agung, Semarang, Indonesia

- Kartika Firdausy, Universitas Ahmad Dahlan, Yogyakarta, Indonesia

- Siti Nurmaini, Universitas Sriwijaya, Palembang, Indonesia

\section{General Chairs}

- Tole Sutikno, Universitas Ahmad Dahlan, Yogyakarta, Indonesia

- Suharyanto, Universitas Gadjah Mada, Yogyakarta, Indonesia

- Deris Stiawan, Universitas Sriwijaya, Palembang, Indonesia

\section{Finance and Treasurer}

- Wiwiek Fatmawati, Universitas Islam Sultan Agung, Semarang, Indonesia

- Lina Handayani, Universitas Ahmad Dahlan, Yogyakarta, Indonesia

\section{Publicity}

- Son Ali Akbar, Universitas Ahmad Dahlan, Yogyakarta, Indonesia

- Muhammad Qomaruddin, Universitas Islam Sultan Agung, Semarang, Indonesia

- Sam F Chaerul, Universitas Islam Sultan Agung, Semarang, Indonesia

- Ahmad Heryanto, Universitas Sriwijaya, Palembang, Indonesia

\section{General co-Chairs}

- I Wayan Mustika, IEEE Indonesia Section

- Imam Much Ibnu Subroto, Universitas Islam Sultan Agung, Semarang, Indonesia

- Zulfatman, Universitas Muhammadiyah Malang, Malang, Indonesia

- Krisna Adiyarta, Universitas Budi Luhur, Jakarta, Indonesia

\section{Publication}

- Balza Achmad, Universitas Gadjah Mada, Yogyakarta, Indonesia

- Mochammad Facta, Universitas Diponegoro, Semarang, Indonesia

\section{Public Relations}

- Aina Musdholifah, Universitas Gadjah Mada, Yogyakarta, Indonesia

- Arief Marwanto, Universitas Islam Sultan Agung, Semarang, Indonesia

- Muhammad Syafrullah, Universitas Budi Luhur, Jakarta, Indonesia

- Riky Dwi Puriyanto, Universitas Ahmad Dahlan, Yogyakarta, Indonesia 


\section{Technical Program Committee}

\section{Technical Program Committee}

\begin{tabular}{|c|c|c|}
\hline Rajan A & Tata Consultancy Services & India \\
\hline Ali Abbas & $\begin{array}{l}\text { HIAST Higher Institute for Applied Science and } \\
\text { Technology }\end{array}$ & Syria \\
\hline Hany Abd El-Aal & Cairo University & Egypt \\
\hline Saied Abd El-atty & Menoufia University-Faculty of Electronic Engineering & Egypt \\
\hline Malaoui Abdessamad & Sultan Moulay Slimane University of Beni Mellal & Morocco \\
\hline Fairul Azhar Abdul Shukor & Universiti Teknikal Malaysia Melaka & Malaysia \\
\hline Balza Achmad & Universitas Gadjah Mada (Indonesia) & Indonesia \\
\hline Muhammad Ishtiaq Ahmad & Beijing Institute of Technology & P.R. China \\
\hline Hamid Alasadi & IRAQ- BASRA & Iraq \\
\hline Mohammed Alghamdi & Al-Baha University & Saudi Arabia \\
\hline Mehran Alidoost Nia & University of Tehran & Iran \\
\hline Gholamreza Alirezaei & RWTH Aachen University & Germany \\
\hline Yeasir Arafat & Bangladesh University of Engineering and Technology & Bangladesh \\
\hline Diego Arcos-Aviles & Universidad de las Fuerzas Armadas ESPE & Ecuador \\
\hline Ramy Atawia & Queen's University & Canada \\
\hline Shakti Awaghad & GHRCE, Nagpur & India \\
\hline Eduard Babulak & Fort Hays State University & USA \\
\hline Peter Balazs & Austrian Academy of Sciences & Austria \\
\hline Marco Baldi & Università Politecnica delle Marche & Italy \\
\hline $\begin{array}{l}\text { Herath Mudiyanselage Nelanga } \\
\text { Bandara }\end{array}$ & University of Moratuwa & Sri Lanka \\
\hline Ihsen Ben Mbarek & National Engineering School of Tunis & Tunisia \\
\hline Alper Bereketli & ASELSAN Inc. & Turkey \\
\hline Tuğçe Bilen & Istanbul Technical University & Turkey \\
\hline Rodrigo Campos Bortoletto & $\begin{array}{l}\text { São Paulo Federal Institute of Education, Science and } \\
\text { Technology }\end{array}$ & Brazil \\
\hline Yue Cao & Northumbria University & $\begin{array}{l}\text { United Kingdom (Great } \\
\text { Britain) }\end{array}$ \\
\hline Maria Chiara Caschera & CNR & Italy \\
\hline Arcangelo Castiglione & University of Salerno & Italy \\
\hline Suryadip Chakraborty & Johnson C. Smith University & USA \\
\hline Parag Chatterjee & National Technological University, Buenos Aires & Argentina \\
\hline Di Chen & University of Rostock & Germany \\
\hline Adilson Chinatto & Espectro Ltd. & Brazil \\
\hline Salim Chitroub & Electronics and Computer Science Faculty, USTHB & Algeria \\
\hline B Chitti Babu & The University of Nottingham Malaysia Campus & Malaysia \\
\hline Domenico Ciuonzo & $\begin{array}{l}\text { Network Measurement and Monitoring (NM2), Naples, } \\
\text { IT }\end{array}$ & Italy \\
\hline Paolo Crippa & Università Politecnica delle Marche & Italy \\
\hline Sanjoy Debbarma & National Institute of Technology Meghalaya & India \\
\hline George Dekoulis & Aerospace Engineering Institute & Cyprus \\
\hline July Katherine Díaz Barriga & Universidad Distrital Francisco José de Caldas & Colombia \\
\hline Saurabh Dixit & Babu Banarsi Das University, Lucknow & India \\
\hline Noha El-Ganainy & Arab Academy of Science and Technology AAST & Egypt \\
\hline Mochammad Facta & Diponegoro University & Indonesia \\
\hline Wei Feng & Tsinghua University & P.R. China \\
\hline Andrea Fiaschetti & University of Rome "La Sapienza" & Italy \\
\hline Muftah Fraifer & IDC-CSIS-UL & Ireland \\
\hline Felix J. Garcia Clemente & University of Murcia & Spain \\
\hline Hamza Ghandorh & University of Western Ontario & Canada \\
\hline Wajeb Gharibi & Jazan University, KSA & Saudi Arabia \\
\hline Srideep Ghosh & ELTRON Wireless & India \\
\hline Syed Ameer Ahmed Gillani & Zhejiang University & P.R. China \\
\hline
\end{tabular}


Visvasuresh Victor

Govindaswamy

Lacrimioara Grama

Henry Griffith

Burhan Gulbahar

Berkin Güler

Rohit Gupta

Zulfatman Has

Meisam Hashemi

Sherief Hashima

Jun $\mathrm{He}$

Ahmed Helmy

A. K. M. Mahtab Hossain

Zhaozheng $\mathrm{Hu}$

Tilokchan Irengbam

Md. Moidul Islam

Anish Jindal

Mohammed Kaabar

Murali Krishna Kadiyala

Dimitrios Kallergis

Jyotirmoy Karjee

Inderpreet Kaur

Shanu Khan

Yassine Khlifi

Jens Klare

Bo Kong

Fukuro Koshiji

Deepika Koundal

Sunil Kumar

Takashi Kurimoto

Chia-Hung Lai

Jia-Han Li

Xiangguo Li

Xiaojun Li

David Luengo

Shahid Manzoor

Rajeev Mathur

Sukadev Meher

Ahmed Mobashsher

Ratan Kumar Mondal

Rodrigo Montufar-Chaveznava

Martin Mudroch

Bodhibrata Mukhopadhyay

Ronald Mulinde

Fernando Mussoi

Nagendra Kumar Nainar

Farid Naït-Abdesselam

Abdellah Najid

Amir Nakib

Syed Mohsen Naqvi

Joanna Isabelle Olszewska

Feng Ouyang

Michel Owayjan

Oskars Ozolins
Concordia University

USA

Technical University of Cluj-Napoca

Michigan State University

Ozyegin University

Koc University

Thapar University

University of Muhammadiyah Malang

University of Isfahan

Engineering dept, Nuclear Research Center, EAEA,

Cairo

University of New Brunswick

Qualcomm Technologies Inc.

University of Greenwich

Georgia Institute of Technology

Manipur University

Friedrich Schiller University Jena

Thapar University, Patiala (India)

Washington State University

Cisco Systems. Inc

University of Piraeus

Tata Consultancy Services

Chandigarh University, Mohali

AICTE

Umm Al-Qura University, KSA

Fraunhofer FHR

PLA University of Science and Technology

Tokyo Polytechnic University

Chitkara University

The LNM Institute of Information Technology, Jaipur

NII

National Cheng Kung University

National Taiwan University

Henan University of Technology

Texas A\&M University

Universidad Politecnica de Madrid (UPM)

UCSI Universiti Kuala Lumpur

Geetanjali Instt of Tech Studies, Udaipur

National Institute of Technology, Rourkela

The University of Queensland

Queensland University of Technology

Facultad de Ingeniería, Universidad Nacional

Autonoma de Mexico

Czech Technical University in Prague

Indian Institute of Technology Delhi

University of South Australia

Federal Institute of Santa Catarina

CISCO

Paris Descartes University

Institut National des Postes et Télécommunications

University Paris East

Newcastle University

University of Gloucestershire

Johns Hopkins University / Applied Physics Lab

American University of Science \& Technology

RISE Acreo
Romania

USA

Turkey

Turkey

India

Indonesia

Iran

Egypt

Canada

USA

United Kingdom (Great

Britain)

USA

India

Germany

India

USA

USA

Greece

India

India

India

Saudi Arabia

Germany

P.R. China

Japan

India

India

Japan

Taiwan

Taiwan

P.R. China

USA

Spain

Malaysia

India

India

Australia

Australia

Mexico

Czech Republic

India

Australia

Brazil

USA

France

Morocco

France

United Kingdom (Great

Britain)

United Kingdom (Great

Britain)

USA

Lebanon

Sweden 
Shashikant Patil

Shashikant Patil

Gabriele Piantadosi

Rajesh Pindoriya

Octavian Postolache

Adhi Prahara

Nadia Qasim

Harikumar Rajaguru

Shuvendu Rana

Hemant Kumar Rath

Muhammad Raza

Mohamed Rehan

Abdalhossein Rezai

Munawar Riyadi

Indra Riyanto

Olympia Roeva

Karla Maria Ronquillo Gonzalez

Muthukumar S

Zulhisyam Salleh

Aratã Saraiva

Gnane Swarnadh Satapathi

Hans Schotten

Ljiljana Šerić

Aditi Sharma

Satish Sharma

Rupesh Singh

Saurabh Srivastava

Deris Stiawan

Ravi Subban

Imam Much Ibnu Subroto

Hengky Susanto

TH Sutikno

Tole Sutikno

Srinivasulu Tadisetty

Pooya Taheri

Min Keng Tan

Xuanxuan Tang

Revak Tyagi

Sudhanshu Tyagi

Dimitris Varoutas

Marcel Wagner

Hao Wu

Kun-Da Wu

Kishore Yalamanchili

Mohammed Younis

Anton Yudhana

Pujianto Yugopuspito

Nemanja Zdravkovic

Chi Zhang

Zhe Zhang

Quanxin Zhao

Jing Zhou

Tudor Cătălin Zorilă
SVKMs NMiMS Mumbai India

SVKM NMIMS Mumbai India

University of Naples Federico II

Indian Institute of Technology Mandi

Instituto de Telecomunicações, Lisboa/IT

Universitas Ahmad Dahlan

King's College London

anna University Chennai

University of Strathclyde

Tata Consultancy Services

HUST Wuhan China

AvidBeam Technlogies

ACECR

Diponegoro University

Universitas Budi Luhur

Institute of Biophysics and Biomedical Engineering

Universidad Tecnológica de Chihuahua

Indian Institute of Information Technology, Tamilnadu

Politeknik Melaka

UESPI

National Institute of Technology, Karnataka

University of Kaiserslautern

University of Split - Faculty of El. Eng., Mech. Eng.

and Naval Arch.

MBM Engineering College Jodhpur

ISRO Satellite Centre

Tantia University

NIt $\mathrm{K}$

University of Sriwijaya

Pondicherry University, Pondicherry

Universitas Islam Sultan Agung

Huawei Technology

Institute of Advanced Engineering and Science

Universitas Ahmad Dahlan

Kakatiya University College of Engineering and

Technology

SFU

Universiti Malaysia Sabah

PLA Army Engineering University

Cisco Systems

Thapar University, Patiala

University of Athens

University of São Paulo

ZTE Corporation

HTC Corporation

Google

University of Baghdad

Ahmad Dahlan University

Universitas Pelita Harapan

Norwegian University of Science and Techonlogy

Midea America Corporation

Geroge Mason University

University of Electronic Science and Technology of

China

University of Science and Technology of China

Toshiba Cambridge Research Laboratory
India

India

Italy

India

Portugal

Indonesia

United Kingdom (Great

Britain)

India

United Kingdom (Great

Britain)

India

P.R. China

Egypt

Iran

Indonesia

Indonesia

Bulgaria

Mexico

India

Malaysia

Brazil

India

Germany

Croatia

India

India

India

India

Indonesia

India

Indonesia

Hong Kong

Indonesia

Indonesia

India

Canada

Malaysia

P.R. China

USA

India

Greece

Brazil

P.R. China

Taiwan

USA

Iraq

Indonesia

Indonesia

Norway

USA

USA

P.R. China

P.R. China

United Kingdom (Great

Britain) 


\section{TABLE OF CONTENTS}

\begin{tabular}{|c|c|}
\hline $\begin{array}{l}\text { High Performance Direct Torque Control of Induction Motor Drives: } \\
\text { Problems and Improvements } \\
\text { Nik Rumzi Nik Idris (UTM-PROTON Future Drive Laboratory, Universiti } \\
\text { Teknologi Malaysia, Johor, Malaysia), Tole Sutikno (Department of Electrical } \\
\text { Engineering, Universitas Ahmad Dahlan, Yogyakarta, Indonesia) }\end{array}$ & 1 \\
\hline $\begin{array}{l}\text { Towards Development of A Computerised System for Screening and } \\
\text { Monitoring of Diabetic Retinopathy } \\
\text { Hanung Adi Nugroho (Universitas Gadjah Mada, Indonesia) }\end{array}$ & 8 \\
\hline $\begin{array}{l}\text { Performance Analysis of Network Emulator Based On The Use Of } \\
\text { Resources In Virtual Laboratory } \\
\text { Yuri Ariyanto, Yan Watequlis Syaifudin, Budi Harijanto (State Polytechnic of } \\
\text { Malang,Malang, East Java, Indonesia) }\end{array}$ & 9 \\
\hline $\begin{array}{l}\text { Teaching And Learning Support For Computer Architecture And } \\
\text { Organization Courses Design On Computer Engineering and Computer } \\
\text { Science For Undergraduate: A Review } \\
\text { Wijaya Kurniawan,Mochammad Hannats Hanafi Ichsan (Brawijaya University, } \\
\text { Malang, East Java, Indonesia) }\end{array}$ & 15 \\
\hline $\begin{array}{l}\text { WatsaQ: Repository of Al Hadith in Bahasa (Case Study: Hadith Bukhari) } \\
\text { Atqia Aulia, Dewi Khairani, Rizal Broer Bahaweres, and Nashrul Hakiem } \\
\text { (Department of Informatics, UIN Syarif Hidayatullah, Jakarta, Indonesia) }\end{array}$ & 21 \\
\hline $\begin{array}{l}\text { IoT Smart Device for e-Learning Content Sharing on Hybrid Cloud } \\
\text { Environment } \\
\text { Mohd. Yazid Idris, Deris Stiawan, Nik Mohd Habibullah, Abdul Hadi Fikri, Mohd } \\
\text { Rozaini Abd Rahim, Massolehin Dasuki (Universiti Teknologi Malaysia, Johor } \\
\text { Bahru, Malaysia) }\end{array}$ & 25 \\
\hline $\begin{array}{l}\text { Target Tracking in Mobile Robot under Uncertain Environment using } \\
\text { Fuzzy Logic Controller } \\
\text { Ade Silvia Handayani, Tresna Dewi, Nyayu Latifah Husni (State Polytechnic of } \\
\text { Sriwijaya,Palembang, Indonesia), Siti Nurmaini, Irsyadi Yani (University of } \\
\text { Sriwijaya, Palembang, Indonesia) }\end{array}$ & 30 \\
\hline $\begin{array}{l}\text { Nitrogen (N) Fertilizer Measuring Instrument On Maize-Based Plant } \\
\text { Microcontroller } \\
\text { Hendra Yufit Riskiawan, Taufiq Rizaldi, Dwi Putro S. Setyohadi, Tri Leksono } \\
\text { (Information Technology Department, Politeknik Negeri Jember, Indonesia) }\end{array}$ & 35 \\
\hline $\begin{array}{l}\text { Reconfigurable Logic Embedded Architecture of Support Vector Machine } \\
\text { Linear Kernel } \\
\text { Jeevan Sirkunan, N. Shaikh-Husin and M. N. Marsono (Fac. of Electrical Eng., } \\
\text { Universiti Teknologi Malaysia, Johor, Malaysia), Trias Andromeda (Diponegoro } \\
\text { University, Semarang, Indonesia) }\end{array}$ & 39 \\
\hline
\end{tabular}




\begin{tabular}{|c|c|}
\hline $\begin{array}{l}\text { An Analysis of Concentration Region on Powerpoint Slides using Eye } \\
\text { Tracking } \\
\text { Fergyanto E. Gunawan, Oky Wijaya, Benfano Soewito, Sevenpri Candra Diana } \\
\text { (Bina Nusantara University, Jakarta, Indonesia) Cosmas E. Suharyanto( Putera } \\
\text { Batam University, Riau Archipelago, Indonesia) }\end{array}$ & 44 \\
\hline $\begin{array}{l}\text { Implementation of K-Means Clustering Method to Distribution of High } \\
\text { School Teachers } \\
\text { Triyanna Widiyaningtyas, Martin Indra Wisnu Prabowo, M. Ardhika Mulya } \\
\text { Pratama (Electrical Engineering Departement, Universitas Negeri Malang, } \\
\text { Malang, Indonesia) }\end{array}$ & 49 \\
\hline $\begin{array}{l}\text { Incremental High Throughput Network Traffic Classifier } \\
\text { H. R. Loo, Alireza Monemi, and M. N. Marsono (Faculty of Electrical } \\
\text { Engineering, Universiti Teknologi Malaysia, Johor, Malaysia), Trias Andromeda } \\
\text { ( Diponegoro University, Semarang, Indonesia) }\end{array}$ & 55 \\
\hline $\begin{array}{l}\text { Edge Detection on Objects of Medical Image with Enhancement multiple } \\
\text { Morphological Gradient Method } \\
\text { Jufriadif Na`am (Computer Science Faculty, Universitas Putra Indonesia YPTK, } \\
\text { Padang, Indonesia) }\end{array}$ & 61 \\
\hline $\begin{array}{l}\text { Unified Concept-based Multimedia Information Retrieval Technique } \\
\text { Ridwan Andi Kambau, Zainal Arifin Hasibuan (Faculty of Computer Science, } \\
\text { University of Indonesia, Depok, West Java, Indonesia) }\end{array}$ & 68 \\
\hline $\begin{array}{l}\text { Text Modeling In Adaptive Educational Chat Room Based On Madamira } \\
\text { Tool } \\
\text { Jehad A. H. Hammad, Mochamad Hariadi, Mauridhi Hery Purnomo } \\
\text { (Department of Computer Engineering, Institut Teknologi Sepuluh Nopember } \\
\text { (ITS) Surabaya, Indonesia), Nidal A. M Jabari (Department of Computer, } \\
\text { Technical Colleges(Arroub), Palestine) }\end{array}$ & 76 \\
\hline $\begin{array}{l}\text { Analysis of Statement Branch and Loop Coverage in Software Testing } \\
\text { With Genetic Algorithm } \\
\text { Rizal Broer Bahaweres }{ }^{1,2} \text {, Khoirunnisya Zawawi }{ }^{1} \text {, Dewi Khairani }{ }^{1} \text {, Nashrul } \\
\text { Hakiem }{ }^{1}\left({ }^{1} \text { Department of Informatics, Syarif Hidayatullah State Islamic }\right. \\
\text { University, Jakarta, Indonesia }{ }^{2} \text { Faculty of Computer Science, NRU Higher } \\
\text { School of Economics, Moscow, Russia ) }\end{array}$ & 82 \\
\hline 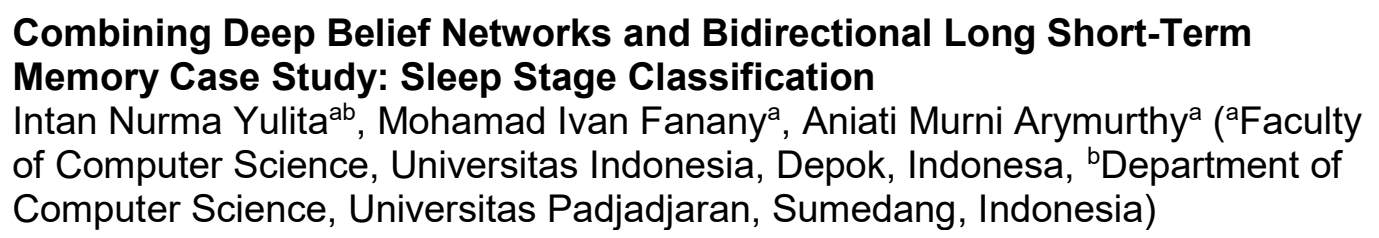 & 88 \\
\hline $\begin{array}{l}\text { Improvement of eGov \& mGov in Multilingual Countries with Digital } \\
\text { Etymology using Sanskrit Grammar } \\
\text { Arijit Das }\end{array}$ & 91 \\
\hline
\end{tabular}




\begin{tabular}{|c|c|}
\hline $\begin{array}{l}\text { EEG Based Emotion Monitoring Using Wavelet and Learning Vector } \\
\text { Quantization } \\
\text { Esmeralda C. Djamal and Poppi Lodaya (Universitas Jenderal Achmad Yani, } \\
\text { Bandung, Indonesia) }\end{array}$ & 94 \\
\hline $\begin{array}{l}\text { Myoelectric control systems for hand rehabilitation device: a review } \\
\text { Khairul Anam, Ahmad Adib Rosyadi, Bambang Sujanarko (University of } \\
\text { Jember, Jember, Indonesia), Adel Al-Jumaily (School of Biomedical } \\
\text { Engineering, University of Technology, Sydney, Australia) }\end{array}$ & 100 \\
\hline $\begin{array}{l}\text { Variance Analysis of Photoplethysmography for Blood Pressure } \\
\text { Measurement } \\
\text { Hendrana Tjahjadi, Kalamullah Ramli (Departement of Electrical Engineering, } \\
\text { Universitas Indonesia, Depok, Indonesia) }\end{array}$ & 106 \\
\hline $\begin{array}{l}\text { Implementation of Unbiased Stereology Method for Organ Volume } \\
\text { Estimation using Image Processing }\end{array}$ & 110 \\
\hline $\begin{array}{l}\text { Mohammad Ammar Faiq, Balza Achmad, Ginus Partadiredja (Universitas Gadjah } \\
\text { Mada Yogyakarta, Indonesia) }\end{array}$ & \\
\hline $\begin{array}{l}\text { Ethnobotany Database: Exploring diversity medicinal plants of Dayak } \\
\text { Tribe Borneo } \\
\text { Haeruddin }{ }^{1} \text {, Ummul Hairah }{ }^{1} \text {, Edy Budiman }{ }^{1} \text {, Herni Johan (Department of } \\
\text { Computer Science and Information Technology, Universitas Mulawarman } \\
\text { Samarinda - Indonesia, }{ }^{2} \text { Departement of Mutiara Mahakam Academy of } \\
\text { Midwifery, AKBID Samarinda, Samarinda - Indonesia) }\end{array}$ & 116 \\
\hline $\begin{array}{l}\text { Automated Post-Trabeculectomy Bleb Assesment by Using Image } \\
\text { Processing }\end{array}$ & 122 \\
\hline $\begin{array}{l}\text { Agwin Fahmi Fahanani, Hasballah Zakaria, Andika Prahasta, Elsa Gustianty, R. } \\
\text { Maula Rifada, Astrid Chairini (Department of Biomedical Engineering, Institut } \\
\text { Teknologi Bandung, Bandung, Indonesia) }\end{array}$ & \\
\hline Non-invasive Hemoglobin Measurement for Anemia Diagnosis & 125 \\
\hline $\begin{array}{l}\text { Raditya Artha Rochmanto, Hasballah Zakaria, Ratih Devi Alviana, Nurhalim } \\
\text { Shahib ('Department of Biomedical Engineering, Institut Teknologi Bandung, } \\
{ }^{2} \text { Medical Faculty Padjajaran University Bandung, Indonesia Bandung, } \\
\text { Indonesia) }\end{array}$ & \\
\hline $\begin{array}{l}\text { Poincaré plot of fingertip photoplethysmogram pulse amplitude suitable } \\
\text { to assess diabetes status }\end{array}$ & 130 \\
\hline $\begin{array}{l}\text { Bagus Haryadi }{ }^{1,2} \text {, Lin, Gen-Min }{ }^{2} ; \text { Yang, Chieh-Ming }{ }^{2} \text {; Chu, Shiao- Chiang }{ }^{2} ; \text { Wu, } \\
\text { Hsien-Tsai ( Department of Electrical Engineering National Dong-Hwa } \\
\text { University Hualien, Taiwan) }\end{array}$ & \\
\hline $\begin{array}{l}\text { Certain Factor Analysis for Extra Pulmonary Tuberculosis Diagnosis } \\
\text { Ramadiani, Nur Aini, Heliza Rahmania Hatta, Fahrul Agus, Zainal Ariffin, Azainil } \\
\text { (Mulawarman University, Samarinda, Indonesia) }\end{array}$ & 134 \\
\hline
\end{tabular}




\begin{tabular}{|c|c|}
\hline $\begin{array}{l}\text { The Improvement of Phonocardiograph Signal (PCG) Representation } \\
\text { Through the Electronic Stethoscope } \\
\text { Sumarna, Juli Astono, Agus Purwanto, Dyah Kurniawati Agustika (Universitas } \\
\text { Negeri Yogyakarta) }\end{array}$ & 141 \\
\hline $\begin{array}{l}\text { Neural Network on Mortality Prediction for the Patient Admitted } \\
\text { with ADHF (Acute Decompensated Heart Failure) } \\
\text { M. Haider Abu Yazid, Shukor Talib, Muhammad Haikal Satria (Universiti } \\
\text { Teknologi Malaysia (UTM), Johor Bahru, Malaysia) Azmee Abd Ghazi (National } \\
\text { Heart Institute (IJN), Kuala Lumpur, Malaysia) }\end{array}$ & 146 \\
\hline $\begin{array}{l}\text { Measurement Of Maximum Value Of Dental Radiograph To Predict The } \\
\text { Bone Mineral Density }\end{array}$ & 152 \\
\hline $\begin{array}{l}\text { Sri Lestari, Mohammad Diqi (Faculty of Science and Technology, UNRIYO } \\
\text { Yogyakarta, Indonesia), Rini Widyaningrum (Departement of Dentomaxillofacial } \\
\text { Radiology Faculty of Dentistry, Universitas Gadjah Mada Yogyakarta, } \\
\text { Indonesia) }\end{array}$ & \\
\hline $\begin{array}{l}\text { Feature Extraction and Classification of Thorax X-Ray Image in the } \\
\text { Assessment of Osteoporosis }\end{array}$ & 156 \\
\hline $\begin{array}{l}\text { Riandini, Mera Kartika Delimayanti (Politeknik Negeri Jakarta Kampus UI } \\
\text { Depok West Java) }\end{array}$ & \\
\hline $\begin{array}{l}\text { 2D-Sigmoid Enhancement Prior to Segment MRI Glioma Tumour Pre } \\
\text { Image-Processing }\end{array}$ & 161 \\
\hline $\begin{array}{l}\text { Setyawan Widyarto, Siti Rafidah Binti Kassim (Faculty of Communication, } \\
\text { Visual Art and Computing, UNISEL, Kuala Selangor, Malaysia), Widya Kumala } \\
\text { Sari (Alumni of Faculty of Medicine, Universitas Gadjah Mada Indonesia) }\end{array}$ & \\
\hline $\begin{array}{l}\text { Alerting System for Sport Activity Based on ECG Signals using } \\
\text { Proportional Integral Derivative }\end{array}$ & 166 \\
\hline $\begin{array}{l}\text { Vika Octaviani, Arief Kurniawan, Yoyon Kusnendar Suprapto, Ahmad Zaini } \\
\text { (Institut Teknologi Sepuluh Nopember, Surabaya Indonesia) }\end{array}$ & \\
\hline $\begin{array}{l}\text { Design of Automatic Switching Bio-Impedance Analysis (BIA) for Body Fat } \\
\text { Measurement }\end{array}$ & 172 \\
\hline $\begin{array}{l}\text { Munawar A Riyadi, Achmad Ngaqib Muthouwali, Teguh Prakoso (Department } \\
\text { of Electrical Engineering, Diponegoro University, Semarang, Indonesia) }\end{array}$ & \\
\hline $\begin{array}{l}\text { Precise Wide Baseline Stereo Image Matching for Compact Digital } \\
\text { Cameras } \\
\text { Martinus Edwin Tjahjadi, Fourry Handoko (National Institute of Technology } \\
\text { (ITN) Malang, Malang, Indonesia) }\end{array}$ & 177 \\
\hline $\begin{array}{l}\text { Robust and Imperceptible Image Watermarking by DC Coefficients Using } \\
\text { Singular Value Decomposition }\end{array}$ & 183 \\
\hline
\end{tabular}


Christy Atika Sari, Eko Hari Rachmawanto, De Rosal Ignatius Moses Setiadi

(Dian Nuswantoro University (UDINUS), Semarang, Indonesia)

Region of Interest Detection for Pregnancy Image Processing

M. Khairudin, Joko Laras B T, Dessy Irmawati (Universitas Negeri Yogyakarta, Yogyakarta,Indonesia)

Shape Defect Detection for Product Quality Inspection and monitoring System

Norhashimah Mohd Saad ${ }^{1}$, Nor Nabilah Syazana Abdul Rahman ${ }^{1}$, Abdul Rahim Abdullah( Universiti Teknikal Malaysia Melaka, Durian Tunggal, Melaka), Farhan Abdul Wahab (Infineon Technologies Sdn. Bhd, Batu Berendam, Melaka)

Toward a New Approach in Fruit Recognition using Hybrid RGBD Features and Fruit Hierarchy Property

Ema Rachmawati, Iping Supriana, Masayu Leylia Khodra (School of Electrical Engineering and Informatics, Institut Teknologi Bandung)

Mobile Content Based Image Retrieval Architectures

Arif Rahman (Universitas Ahmad Dahlan), Edi Winarko, Moh. Edi Wibowo (Universitas Gadjah Mada, Yogyakarta, Indonesia)

Computer Vision Based Object Tracking as a Teaching Aid for High School Physics Experiments

G.D. Illeperuma (The Open University of Sri Lanka, Nawala, Nugegoda, Sri Lanka), D.U.J. Sonnadara(University of Colombo, Sri Lanka)

Texture Analysis and Fracture Identification of Lower Extremity Bones XRay Images

Rahayu Suci Prihatini, Anif Hanifa Setyaningrum, Imam Marzuki Shofi (Departement of Informatics Engineering, UIN Syarif Hidayatullah, Jakarta, Indonesia)

Analysis of the Indonesian Vowel /e/ For Lip Synchronization Animation Anung Rachman, Risanuri Hidayat, Hanung Adi Nugroho (Universitas Gadjah Mada, Yogyakarta, Indonesia)

\section{Anti-Cheating Presence System Based on 3WPCA- Dual Vision Face} Recognition

Edy Winarno, Wiwien Hadikurniawati, Imam Husni Al Amin, Muji Sukur (Faculty of Information Technology, Universitas Stikubank Semarang Indonesia)

Sketch Plus Colorization Deep Convolutional Neural Networks for Photos Generation from Sketches

Vinnia Kemala Putri and Mohamad Ivan Fanany (Faculty of Computer Science, Universitas Indonesia, Depok, West-Java Indonesia)

Imperceptible Image Watermarking based on Chinese Remainder Theorem over the Edges 


\begin{tabular}{|c|c|}
\hline $\begin{array}{l}\text { Informatics Engineering Universitas Dian Nuswantoro (UDINUS) Semarang, } \\
\text { Indonesia) }\end{array}$ & \\
\hline $\begin{array}{l}\text { Wood Texture Detection with Conjugate Gradient Neural Network } \\
\text { Algorithm } \\
\text { Setyawan Widyarto, I Nyoman Suryasa, Otto Fajarianto (Universitas Budi } \\
\text { Luhur, Jakarta, Indonesia), Mohd Shafry Mohd Rahim (Universiti Teknologi } \\
\text { Malaysia, Johor Bahru, Malaysia), Khairul Annuar bin Abdullah (Universiti } \\
\text { Selangor, Malaysia), Gigih Priyandoko, Gilang Anggit Budaya (Universiti } \\
\text { Malaysia Pahang, Malaysia) }\end{array}$ & 240 \\
\hline $\begin{array}{l}\text { Spoken Word Recognition Using MFCC and Learning Vector Quantization } \\
\text { Esmeralda C. Djamal, Neneng Nurhamidah and Ridwan Ilyas (Universitas } \\
\text { Jenderal Achmad Yani, Bandung, Indonesia) }\end{array}$ & 246 \\
\hline $\begin{array}{l}\text { A Hierarchical Description-based Video Monitoring System for Elderly } \\
\text { Mochamad Irwan Nari, Agung Wahyu Setiawan and Widyawardana Adiprawita } \\
\text { (Institut Teknologi Bandung, Indonesia) }\end{array}$ & 252 \\
\hline $\begin{array}{l}\text { Performance Measurement Based on Coloured Petri Net Simulation of } \\
\text { Scalable Business Processes } \\
\text { Abd. Charis Fauzan, Riyanarto Sarno, Muhammad Ainul Yaqin (Institut } \\
\text { Teknologi Sepuluh Nopember, Surabaya, Indonesia) }\end{array}$ & 257 \\
\hline $\begin{array}{l}\text { The Design a System of Retention and Control on Broiler Farms Based on } \\
\text { The Flow of Data } \\
\text { Ahmad Sanmorino, Isabella (Universitas Indo Global Mandiri, Palembang, } \\
\text { Indonesia) }\end{array}$ & 263 \\
\hline $\begin{array}{l}\text { Empirical Investigation on Factors Related to Individual of Impact } \\
\text { Performance Information System } \\
\text { Tri Lathif Mardi Suryanto, Nur Cahyo Wibowo (Universitas Pembangunan } \\
\text { Nasional “Veteran" Jawa Timur), Djoko Budiyanto Setyohadi(Universitas Atma } \\
\text { Jaya Yogyakarta, Indonesia) }\end{array}$ & 267 \\
\hline $\begin{array}{l}\text { Comparative Study of Web3D Standard Format to Determine the Base } \\
\text { Format for A Web3D Framework } \\
\text { Mursid W. Hananto, Ahmad Ashari, Khabib Mustofa (Universitas Gadjah Mada, } \\
\text { Yogyakarta, Indonesia) }\end{array}$ & 273 \\
\hline $\begin{array}{l}\text { Task-Technology Fit for Textile Cyberpreneur's Intention to Adopt Cloud- } \\
\text { Based M-Retail Application } \\
\text { Nik Zulkarnaen Khidzir, Wan Safra Diyana, Wan Abdul Ghani, Tan Tse Guan } \\
\text { (Faculty of Creative Technology and Heritage, Universiti Malaysia Kelantan, } \\
\text { Bachok, Malaysia), Mohammad Ismail (Faculty of Entrepreneurship and } \\
\text { Business, Universiti Malaysia Kelantan, Kota Bharu, Malaysia) }\end{array}$ & 279 \\
\hline $\begin{array}{l}\text { MAKASSAR SMART CITY OPERATION CENTER PRIORITY OPTIMIZATION } \\
\text { USING FUZZY MULTI-CRITERIA DECISION-MAKING }\end{array}$ & 285 \\
\hline
\end{tabular}




\begin{tabular}{|c|c|}
\hline $\begin{array}{l}\text { Fachrul Kurniawan, Supeno Mardi Susiki Nugroho, Mochamad Hariadi (Institut } \\
\text { Teknologi Sepuluh Nopember (ITS), Surabaya), Aji Prasetya Wibawa } \\
\text { (Universitas Negeri Malang), Munir (Universitas Pendidikan Indonesia, } \\
\text { Bandung, Indonesia) }\end{array}$ & \\
\hline $\begin{array}{l}\text { Ontology-Based Sentence Extraction for Answering Why-Question } \\
\text { A. A. I. N. Eka Karyawati (Department of Computer Science, Faculty of } \\
\text { Mathematics and Natural Sciences, Udayana University, Bali, Indonesia) }\end{array}$ & 290 \\
\hline $\begin{array}{l}\text { The Ontology-Based Methodology Phases To Develop Multi-Agent System } \\
\text { (OmMAS) }\end{array}$ & 296 \\
\hline $\begin{array}{l}\text { Arda Yunianta, Omar Obarukab, Norazah Yusof (King Abdulaziz University, } \\
\text { Rabigh, Saudi Arabia), Aina Musdholifah (Gadjah Mada University, Indonesia), } \\
\text { Nataniel Dengen, Haviluddin (Mulawarman University, Indonesia), Herlina } \\
\text { Jayadiyanti (UPN Veteran Yogyakarta, Indonesia), Mohd Shahizan Othman } \\
\text { (University Teknologi Malaysia, Malaysia). }\end{array}$ & \\
\hline $\begin{array}{l}\text { Scalability Measurement of Business Process Model Using Business } \\
\text { Processes Similarity and Complexity } \\
\text { Muhammad Ainul Yaqin, Riyanarto Sarno, Abd. Charis Fauzan (Informatics } \\
\text { Department, Institut Teknologi Sepuluh Nopember) }\end{array}$ & 302 \\
\hline $\begin{array}{l}\text { Smartphone for Next Generation Attendance System and Human } \\
\text { Resources Payroll System }\end{array}$ & 309 \\
\hline $\begin{array}{l}\text { Benfano Soewito, Fergyanto E. Gunawan (Binus Graduate Programs Bina } \\
\text { Nusantara University Jakarta, Indonesia), Manik Hapsara (University of New } \\
\text { South Wales Canberra, Australia) }\end{array}$ & \\
\hline $\begin{array}{l}\text { Enhancing Online Business Marketing to Expand Market Shares through } \\
\text { IT Governance }\end{array}$ & 315 \\
\hline $\begin{array}{l}\text { Sandy Kosasi, Vedyanto, I Dewa Ayu Eka Yuliani (Information System } \\
\text { Department STMIK Pontianak Pontianak, West Kalimantan, Indonesia) }\end{array}$ & \\
\hline A Generic Framework for Information Security Policy Development & 320 \\
\hline $\begin{array}{l}\text { Wan Basri Wan Ismail, Raja Ahmad Tariqi Raja Ahmad, Setyawan Widyarto } \\
\text { (Faculty of Communication, Visual Art and Computing University of Selangor } \\
\text { Malaysia), Khatipah Abd Ghani (Faculty of Education and Social Science } \\
\text { University of Selangor Malaysia) }\end{array}$ & \\
\hline Modeling IT Value based on Meta-Analysis & 326 \\
\hline $\begin{array}{l}\text { Suhardi, Novianto Budi Kurniawan, Aan Subrata, Jaka Sembiring (School of } \\
\text { Electrical Engineering and Informatics Institut Teknologi Bandung Bandung, } \\
\text { Indonesia) }\end{array}$ & \\
\hline $\begin{array}{l}\text { A Combination of The Evolutionary Tree Miner and Simulated Annealing } \\
\text { Afina Lina Nurlaili, Riyanarto Sarno (Department of Informatics Institut }\end{array}$ & 332 \\
\hline
\end{tabular}




\begin{tabular}{|c|c|}
\hline Teknologi Sepuluh Nopember Surabaya, Indonesia) & \\
\hline $\begin{array}{l}\text { Scalable Attack Analysis of Business Process based on Decision Mining } \\
\text { Classification } \\
\text { Dewi Rahmawati, Riyanarto Sarno (Informatics Department, Institut Teknologi } \\
\text { Sepuluh Nopember, Surabaya, Indonesia) }\end{array}$ & 337 \\
\hline $\begin{array}{l}\text { Service Computing System Engineering Life Cycle } \\
\text { Suhardi, Novianto Budi Kurniawan, Jaka Sembiring (School of Electrical } \\
\text { Engineering and Informatics, Institut Teknologi Bandung,Bandung, Indonesia) }\end{array}$ & 343 \\
\hline $\begin{array}{l}\text { High Efficiency Single Phase Inverter Design } \\
\text { Didi Istardi (Politeknik Negeri Batam, Indonesia) }\end{array}$ & 349 \\
\hline $\begin{array}{l}\text { Analysis of Electric Circuit Model on Atmospheric Pressure Dielectric } \\
\text { Barrier Discharge (DBD) Plasma } \\
\text { Suyadi, Jatmiko E Suseno, Muhammad Nur (Diponegoro University) }\end{array}$ & 352 \\
\hline $\begin{array}{l}\text { COMPRESSED NATURAL GAS (CNG) TECHNOLOGY FOR FUEL POWER } \\
\text { PLANTS } \\
\text { Isworo Pujotomo, Retno Aita Diantari (College of Engineering - PLN } \\
\text { Foundation for Education \& Welfare PT. PLN (Persero) Jakarta, Indonesia) }\end{array}$ & 352 \\
\hline $\begin{array}{l}\text { PID Designs Using DE and PSO Algorithms For Damping Oscillations in a } \\
\text { DC Motor Speed } \\
\text { Lailis Syafaah, Widianto, Ilham Pakaya, Diding Suhardi, M. Irfan (Department of } \\
\text { Electrical Engineering,The University of Muhammadiyah Malang, Indonesia) }\end{array}$ & 354 \\
\hline $\begin{array}{l}\text { Measurement of Partial Discharge Induced Electromagnetic Wave using } \\
\text { Loop Antenna } \\
\text { Umar Khayam, Fendi Imam Fatoni (School of Electrical Engineering and } \\
\text { Informatics, Bandung Institute of Technology, Bandung, Indonesia) }\end{array}$ & 359 \\
\hline $\begin{array}{l}\text { The Effect of Coating on Leakage Current Characteristic of Coast Field } \\
\text { Aged Ceramic Insulator } \\
\text { Dini Fauziah*, Heldi Alfiadi, Rachmawati, Suwarno (School of Electrical } \\
\text { Engineering and Informatics, Institut Teknologi Bandung, Bandung, Indonesia) }\end{array}$ & 363 \\
\hline $\begin{array}{l}\text { Renewable Energy Inclusion on Economic Power Optimization using } \\
\text { Thunderstorm Algorithm }\end{array}$ & 369 \\
\hline $\begin{array}{l}\text { A.N. Afandi (Universitas Negeri Malang, Jawa Timur, Indonesia), Goro Fujita, } \\
\text { Nguyen Phuc Khai (Shibaura Institute of Technology, Tokyo, Japan), Yunis } \\
\text { Sulistyorini (IKIP Budi Utomo, Malang, Indonesia), Nedim Tutkun (Duzce } \\
\text { University,Duzce, Tukey) }\end{array}$ & \\
\hline Optimum Phase Number for Multiphase PWM Inverters & 375 \\
\hline $\begin{array}{l}\text { Anwar Muqorobin, Pekik Argo Dahono and Agus Purwadi (School of Electrical } \\
\text { Engineering and Informatics, Institute of Technology Bandung, Bandung, } \\
\text { Indonesia) }\end{array}$ & \\
\hline
\end{tabular}




\begin{tabular}{|c|c|}
\hline $\begin{array}{l}\text { Small-Disturbance Angle Stability Enhancement using Intelligent Redox } \\
\text { Flow Batteries }\end{array}$ & \multirow[t]{3}{*}{381} \\
\hline $\begin{array}{l}\text { Mohammad Taufik (Padjadjaran University, Sumedang, Indonesia), Dwi } \\
\text { Lastomo (University of PGRI Adi Buana, Surabaya, Indonesia), Herlambang } \\
\text { Setiadi (School of Information Technology \& Electrical Engineering, The } \\
\text { University of Queensland }\end{array}$ & \\
\hline Brisbane, Australia) & \\
\hline $\begin{array}{l}\text { Evaluation Study of Waste Materials for Renewable Energy through 3R } \\
\text { Model in Bogor City }\end{array}$ & \multirow[t]{2}{*}{387} \\
\hline $\begin{array}{l}\text { Didik Notosudjono, Dede Suhendi, Engkos, Bagus Dwi Ramadhon (Electrical } \\
\text { Engineering Department, Universitas Pakuan, Bogor, Indonesia) }\end{array}$ & \\
\hline $\begin{array}{l}\text { Measurement of Partial Discharge inside Metal Enclosed Power Apparatus } \\
\text { using Internal Sensor }\end{array}$ & \multirow[t]{2}{*}{391} \\
\hline $\begin{array}{l}\text { Umar Khayam, Yushan (School of Electrical Engineering and Informatics } \\
\text { Bandung Institute of Technology Bandung, Indonesia) }\end{array}$ & \\
\hline $\begin{array}{l}\text { Design Unmanned Aerial Vehicle Integrated Camera Near Infra-Red to } \\
\text { Observe the Plant Health }\end{array}$ & \multirow[t]{2}{*}{397} \\
\hline $\begin{array}{l}\text { Rizki Wahyu Pratama, Ferry Hadary, Redi Ratiandi Yacoub (Jurusan Teknik } \\
\text { Elektro Fakultas Teknik Universitas Tanjungpura) }\end{array}$ & \\
\hline $\begin{array}{l}\text { Single Frame Resection of Compact Digital Cameras for UAV Imagery } \\
\text { Martinus Edwin Tjahjadi (Department of Geodesy, National Institute of } \\
\text { Technology (ITN) Malang, Indonesia) }\end{array}$ & 401 \\
\hline $\begin{array}{l}\text { A MOVING OBJECTS DETECTION IN UNDERWATER VIDEO USING } \\
\text { SUBTRACTION OF THE BACKGROUND MODEL } \\
\text { M. R. Prabowo, N. Hudayani, S. Purwiyanti, S. R. Sulistiyanti, F. X. A. } \\
\text { Setyawan (Department of Electrical Engineering, Faculty of Engineering } \\
\text { University of Lampung, Bandar Lampung, Indonesia) }\end{array}$ & 406 \\
\hline $\begin{array}{l}\text { Fall Detection Based on Accelerometer and Gyroscope using Back } \\
\text { Propagation } \\
\text { Adlian Jefiza (Institut Teknologi Sepuluh Nopember, Surabaya Indonesia) }\end{array}$ & 410 \\
\hline Honey Yield Prediction Using Tsukamoto Fuzzy Inference System & \multirow[t]{2}{*}{416} \\
\hline $\begin{array}{l}\text { Tri Hastono, Albertus Joko Santoso, Pranowo(Universitas Atma Jaya } \\
\text { Yogyakarta, Indonesia) }\end{array}$ & \\
\hline $\begin{array}{l}\text { Determining The Nutrition of Patient Based on Food Packaging Product } \\
\text { Using Fuzzy C Means Algorithm }\end{array}$ & \multirow[t]{2}{*}{422} \\
\hline $\begin{array}{l}\text { Sri Winiarti, Sri Kusumadewi, Izzati Muhimmah, Herman Yuliansyah } \\
\text { (Universitas Ahmad Dahlan Yogyakarta, Indonesia) }\end{array}$ & \\
\hline
\end{tabular}


The Successful Elements Implementing the eLearning using Cloud Services Data Centre at Private Institution of Higher Learning in Malaysia

Azlinda Abdul Aziz, Setyawan Widyarto, Salyani Osman, Suziyanti Marjudi (Department of Computing, Faculty of Communication, Visual Art and Computing, UNISEL, Kuala Selangor, Malaysia)

Improving E-Book Learning Experience by Learning Recommendation Fergyanto E. Gunawan, Benfano Soewito (Binus Graduate Programs, Bina Nusantara University, Jakarta, Indonesia), and Sevenpri Candra (School of Business Management, Bina Nusantara University, Jakarta, Indonesia).

\section{A Comparison of Cloud Execution Mechanisms: Fog, Edge and Clone Cloud Computing}

Tina Francis (Computer Department, BITS Pilani, Dubai Campus, DIAC Dubai, UAE), Dr. Muthiya Madhiajagan (SCOPE (School of Computer Science and Engineering) VIT University, Vellore Tamil Nadu, India)

\section{Recommendation System on Knowledge Management System via OAl-} PMH

Nyoman Karna, Iping Supriana, Nur Maulidevi (Sekolah Teknik Elektro dan Informatika Institut Teknologi Bandung, Indonesia)

Development and Evaluation of Android Based Notification System to Determine Patient's Medicine for Pharmaceutical Clinic Imam Riadi, Sri Winiarti, Herman Yuliansyah (Department of Informatics Universitas Ahmad Dahlan Yogyakarta)

Implementation of Decision Expert (DEX) in The "SALADGARDEN" Application

Anita Hidayati, Fityan Aula Juyuspan, Cindy Novianty, Muhammad Bima D S (Computer and Informatics Enginering Jakarta State Polytechnic Depok, Indonesia)

Optimizing Effort and Time Parameters of COCOMO II Estimation using Fuzzy Multi-Objective PSO

Kholed Langsari, Riyanarto Sarno (Department of Informatics Engineering Institut Teknologi Sepuluh Nopember Surabaya, Indonesia)

Evaluation Of Knowledge Management System Using Technology Acceptance Model

Jarot S. Suroso, Astari Retnowardhani, Abraham Fernando (Bina Nusantara University Jakarta, Indonesia) advising students' study path 


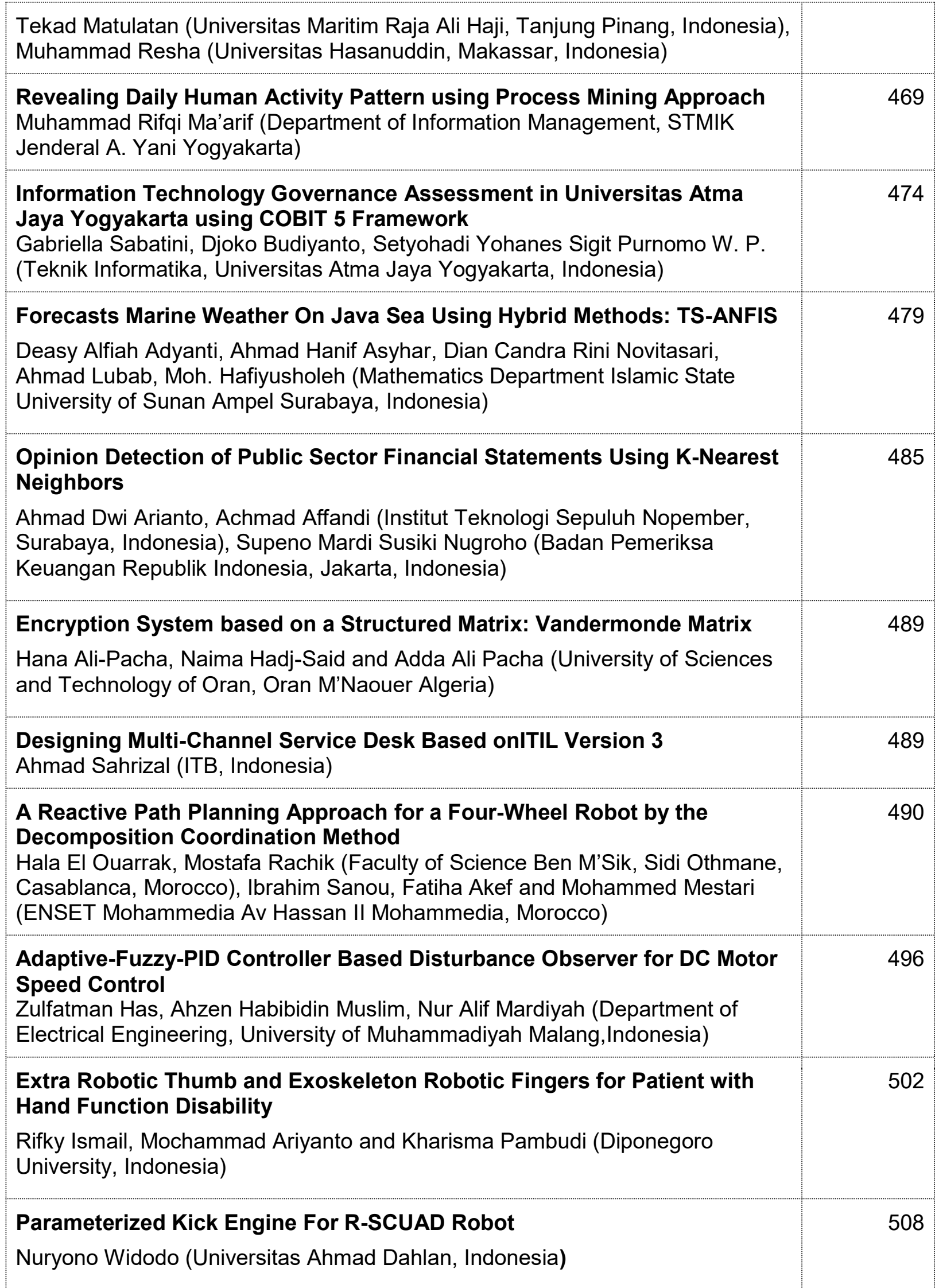




\begin{tabular}{|c|c|}
\hline $\begin{array}{l}\text { Neural Network Controller Design for a Mobile Robot Navigation; a Case } \\
\text { Study } \\
\text { Tresna Dewi, Pola Risma, Yurni Oktarina, and M. Taufik Roseno (Politeknik } \\
\text { Negeri Sriwijaya Palembang, Indonesia) }\end{array}$ & 512 \\
\hline $\begin{array}{l}\text { Redirection Concept of Autonomous Mobile Robot HY-SRF05 Sensor to } \\
\text { Reduce The Number of Sensors } \\
\text { Nuryanto, Andi Widiyanto, Auliya Burhanuddin (Engineering Faculty, } \\
\text { Universitas Muhammadiyah Magelang, Indonesia) }\end{array}$ & 517 \\
\hline $\begin{array}{l}\text { Autonomous Navigation for an Unmanned Aerial Vehicle by the } \\
\text { Decomposition Coordination Method } \\
\text { Chaimaa Jihane } \square \text {, Hala El Ouarrak } \dagger \text {, and Mohamed Mestari } \square \text {, Mostafa } \\
\text { Rachik } \dagger \text { ( } \square \text { Ecole Normale Suprieure d'Enseignement Technique } \\
\text { Mohammedia, Av Hassan II Mohammedia, Morocco, †Faculty of Science Ben } \\
\text { M'Sik, Casablanca, Morocco) }\end{array}$ & 521 \\
\hline $\begin{array}{l}\text { Design of PID Disturbance Observer for Temperature Control on Room } \\
\text { Heating System } \\
\text { Yoga Alif Kurnia Utama (Electronic Engineering Department, University of } \\
\text { Widya Kartika, Surabaya, Indonesia) }\end{array}$ & 527 \\
\hline $\begin{array}{l}\text { Development of Low Cost Supernumerary Robotic Fingers as an Assistive } \\
\text { Device }\end{array}$ & 533 \\
\hline $\begin{array}{l}\text { Mochammad Ariyanto*, Rifky Ismail, Joga Dharma Setiawan, Zainal Arifin } \\
\text { (Department of Mechanical Engineering, Diponegoro University, Semarang, } \\
\text { Indonesia) }\end{array}$ & \\
\hline $\begin{array}{l}\text { Design of A Microchip Optical Switching Driven by Low Direct-Current } \\
\text { Voltage }\end{array}$ & 539 \\
\hline $\begin{array}{l}\text { Dedi Irawan (Islamic State University of Sultan Syarif Kasim Riau, Pekanbaru, } \\
\text { Indonesia) }\end{array}$ & \\
\hline $\begin{array}{l}\text { A Web-Based Wireless Sensor System to Measure Carbon Monoxide } \\
\text { Concentration }\end{array}$ & 544 \\
\hline $\begin{array}{l}\text { Suryono, Ragil Saputra, Bayu Surarso, Ali Bardadi (Diponegoro University, } \\
\text { Semarang, Indonesia) }\end{array}$ & \\
\hline $\begin{array}{l}\text { Detecting the Early Drop of Attention using EEG Signal } \\
\text { Fergyanto E. Gunawan, Krisantus Wanandi, Benfano Soewito (Binus Graduate } \\
\text { Programs, Bina Nusantara University, Jakarta, Indonesia), Sevenpri Candra } \\
\text { (School of Business Management, Bina Nusantara University) }\end{array}$ & 549 \\
\hline $\begin{array}{l}\text { The Design of a Smart Refrigerator Prototype } \\
\text { Z. Ali, S. E. Esmaeili (Department of Electrical and Computer Engineering, } \\
\text { American University of Kuwait, Salmiya, Kuwait) }\end{array}$ & 554 \\
\hline Odor Localization using Gas Sensor for Mobile Robot & 555 \\
\hline
\end{tabular}


Nyayu Latifah Husni, Ade Silvia Handayani (State Polytechnic of Sriwijaya, Palembang, Indonesia), Siti Nurmaini, Irsyadi Yani (University of Sriwijaya, Palembang, Indonesia)

A Project-Based Approach to FPGA-Aided Teaching of Digital Systems Fajar Suryawan (Universitas Muhammadiyah Surakarta, Indonesia)

Performance of Routing Protocol in MANET with Combined Scalable Video Coding

Parma Hadi Rantelinggi, Fridolin Febrianto Paiki (Universitas Papua Manokwari, Indonesia), Kalvein Rantelobo (Universitas Nusa Cendana Kupang, Indonesia)

Attack Scenarios and Security Analysis of MQTT Communication Protocol in loT System

Syaiful Andy, Budi Rahardjo, Bagus Hanindhito (Institut Teknologi Bandung, Bandung, Indonesia)

Encoding of passive anticollision radio-frequency identification surface acoustic waves tags

Alexander Sorokin, Alexander Shepeta, Maurits Wattimena (Major ProblemOriented Computer Complexes Department, State University of Aerospace Instrumentation (SUAI), St. Petersburg, Russia)

Compact Fractal Patch Microstrip Antenna Fed by Coplanar Waveguide for Long Term Evolution Communications Indra Surjati (Universitas Trisakti , Jakarta, Indonesia)

Graphical Approach for RF Amplifier Specification in Radio over Fiber System: Maximum Power Issues

Teguh Prakoso, Munawar Agus Riyadi (Universitas Diponegoro, Semarang, Indonesia), Razali Ngah (Universiti Teknologi Malaysia, Johor Bahru, Malaysia)

FEM Modeling of Squeeze Film Damping Effect in RF-MEMS Switches Syed Turab Haider (Department of Electrical Engineering, National University of Sciences and Technology, Islamabad, Pakistan)

The Onion Routing Performance using Shadow-plugin-TOR Hartanto Kusuma Wardana, Liauw Frediczen Handianto, Banu Wirawan Yohanes* (Faculty of Electronic and Computer Engineering, Universitas Kristen Satya Wacana, Salatiga, Indonesia)

Position Tracking for Static Target using Burst Signals with Time Difference of Arrival Method

Romi Wiryadinata, Alia Shaliha Amany, Imamul Muttakin (Department of Electrical Engineering, University of Sultan Ageng Tirtayasa, Cilegon, Indonesia)

Performance Analysis for MIMO LTE on the High Altitude Platform Station

Catur Budi Waluyo, Yenni Astuti (Department of Electrical Engineering, Sekolah Tinggi Teknologi Adisutjipto, Yogyakarta, Indonesia) 


\begin{tabular}{|c|c|}
\hline $\begin{array}{l}\text { Software Defined Radio Design for OFDM Based Spectrum Exchange } \\
\text { Information Using Arduino UNO and X-Bee }\end{array}$ & \multirow[t]{2}{*}{608} \\
\hline $\begin{array}{l}\text { Arief Marwanto (Univ. Islam Sultan Agung (UNISSULA) Semarang - } \\
\text { Indonesia), Sharifah Kamilah Syed Yusof, Muhammad Haikal Satria (Universiti } \\
\text { Teknologi Malaysia (UTM) Johor Bahru - Malaysia) }\end{array}$ & \\
\hline $\begin{array}{l}\text { Performance Rate for Implementation of Mobile Learning in Network } \\
\text { Edy Budiman, Usfandi Haryaka, Jefferson Roosevelt Watulingas (Universitas } \\
\text { mulawarman, Samarinda - Indonesia), Faza Alameka (Universitas Ahmad } \\
\text { Dahlan, Yogyakarta - Indonesia) }\end{array}$ & 613 \\
\hline $\begin{array}{l}\text { Performance Evaluation of IPv6 Jumbogram Packets Transmission using } \\
\text { Jumbo Frames }\end{array}$ & \multirow[t]{2}{*}{619} \\
\hline $\begin{array}{l}\text { Supriyanto, Rian Sofhan, Rian Fahrizal (Department of Electrical Engineering, } \\
\text { University of Sultan Ageng Tirtayasa, Indonesia), Azlan Osman (School of } \\
\text { Computer Sciences, Universiti Sains Malaysia, Penang, Malaysia) }\end{array}$ & \\
\hline $\begin{array}{l}\text { Performance Analysis of CSI:T Routing in a Delay Tolerant Networks } \\
\text { Hardika Kusuma Putri, Leanna Vidya Yovita, and Ridha Muldina Negara } \\
\text { (Telkom University, Bandung, Indonesia) }\end{array}$ & 624 \\
\hline $\begin{array}{l}\text { A Study of the Number of Wavelengths Impact in the Optical Burst } \\
\text { Switching Core Node }\end{array}$ & \multirow[t]{2}{*}{630} \\
\hline $\begin{array}{l}\text { Hani A. M. Harb (Faculty of CSIT, Baha University, AL-Baha, Saudi Arabia), } \\
\text { Waleed M. Gaballah (Al-Baha Private College of Science, AL-Baha, Saudi } \\
\text { Arabia), Ahmed S. Samra Ahmed Abo-Taleb (Mansoura University, Egypt), } \\
\text { Arief Marwanto (Sultan Agung Islamic Univ. Semarang, Indonesia) }\end{array}$ & \\
\hline $\begin{array}{l}\text { A Reconfigurable MIMO Antenna System for Wireless Communications } \\
\text { Evizal Abdul Kadir (Department of Information Technology, Faculty of } \\
\text { Engineering, Universitas Islam Riau, Pekanbaru, Riau, Indonesia) }\end{array}$ & 634 \\
\hline $\begin{array}{l}\text { Conceptual Framework for Public Policymaking based on System } \\
\text { Dynamics and Big Data } \\
\text { Feldiansyah Bin Bakri Nasution, Nor Erne Nazira Bazin (Universiti Teknologi } \\
\text { Malaysia (UTM) Johor Bahru, Malaysia), Hasanuddin (Faculty of Social and } \\
\text { Politic Sciences, Riau University, Indonesia) }\end{array}$ & 638 \\
\hline $\begin{array}{l}\text { Discovering Process Model from Event Logs by Considering Overlapping } \\
\text { Rules }\end{array}$ & \multirow[t]{2}{*}{645} \\
\hline $\begin{array}{l}\text { Yutika Amelia Effendi, Riyanarto Sarno (Department of Informatics Faculty of } \\
\text { Information Technology, Institut Teknologi Sepuluh Nopember, Surabaya, } \\
\text { Indonesia) }\end{array}$ & \\
\hline
\end{tabular}


CHMM for Discovering Intentional Process Model From Event Logs By Considering Sequence of Activities

Kelly R. Sungkono, Riyanarto Sarno ( Department of Informatics Engineering, Institut Teknologi Sepuluh Nopember, Surabaya, Indonesia)

Sosio-Technical Factors of E-Government Implementation

Darmawan Napitupulu (LIPI, Indonesia); Dana Sensuse (Laboratory of E-

Government, Indonesia); Yudho Sucahyo (University of Indonesia, Indonesia)

\section{Methodology for Constructing Form Ontology}

U. Ungkawa, D. H. Widyantoro \& B. Hendradjaya (School of Electrical Engineering and Informatics, Institut Teknologi Bandung, Bandung, Indonesia)

Integration Protocol Student Academic Information to Campus RFID Gate Pass System

Hendra Gunawan and Evizal Abdul Kadir (Department of Information

Technology, Faculty of Engineering, Universitas Islam Riau, Pekanbaru, Riau, Indonesia)

E-Learning Model for Equivalency Education Program in Indonesia Mesra Betty Yel (Faculty of Computer Science, STIKOM CKI, Jakarta, Indonesia), Sfenrianto (Master in Information Systems Management, Bina Nusantara University, Jakarta, Indonesia)

Developing E-Government Maturity Framework Based on COBIT 5 and Implementing in City Level:Case Study Depok City and South Tangerang City

Fikri Akbarsyah Anza (Public Administration Department, Universitas Indonesia), Dana Indra Sensuse, Arief Ramadhan (Computer Science Department, Universitas Indonesia)

Analysis of Driving Skills based on Deep Learning using Stacked Autoencoders

Takuya Kagawa, Naiwala P. Chandrasiri (Faculty of Information Kogakuin University, Tokyo, Japan)

Minimizing the Estimated Solution Cost with A* Search to Support Minimal Mapping Repair

Inne Gartina Husein*, Benhard Sitohang, Saiful Akbar (Institut Teknologi Bandung)

The rule Extraction of Numerical Association Rule Mining Using Hybrid Evolutionary Algorithm Imam Tahyudin (Kanazawa University, Japan)

Discovering Drugs Combination Pattern Using FP- Growth Algorithm

Rini Anggrainingsih, Nach Rowi Khoirudin, Haryono Setiadi (Informatics Dept Mathematics and Natural Science, UNS Surakarta, Indonesia ) 


\begin{tabular}{|c|c|}
\hline $\begin{array}{l}\text { Classifiers Evaluation: Comparison of Performance Classifiers Based on } \\
\text { Tuples Amount } \\
\text { Mochammad Yusa, Ema Utami (Department of Computer Science, Magister } \\
\text { Teknik Informatika Universitas AMIKOM Yogyakarta, Indonesia) }\end{array}$ & 706 \\
\hline $\begin{array}{l}\text { Prediction of Rupiah Against US Dollar by Using ARIMA } \\
\text { Adiba Qonita, Annas Gading Pertiwi, Triyanna Widiyaningtyas (Electrical } \\
\text { Engineering Department, Universitas Negeri Malang, Malang, Indonesia) }\end{array}$ & 713 \\
\hline $\begin{array}{l}\text { Forming Heterogeneous Group in Cooperative Learning Process using } \\
\text { Partitioning Around Medoids (PAM) and Equitable Distribution } \\
\text { Imam Much Ibnu Subroto, Badieah Assegaf and Wardianto Eko Saputra } \\
\text { (Universitas Islam Sultan Agung, Indonesia) }\end{array}$ & 718 \\
\hline
\end{tabular}




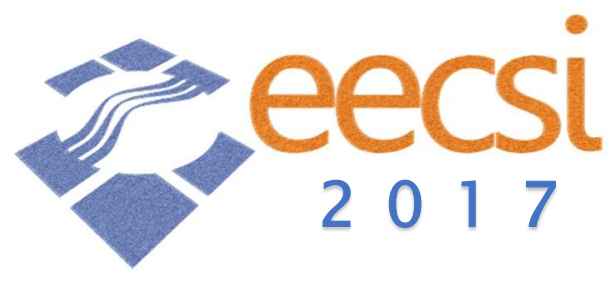

\title{
PROCEEDINGS
}

\section{4th International Conference on Electrical Engineering, Computer Science and Informatics (EECSI)}

19-21 September 2017, Yogyakarta, Indonesia

\author{
Editors: \\ Munawar A Riyadi \\ Mochammad Facta \\ Deris Stiawan \\ Hatib Rahmawan
}




\section{Proceedings}

\section{4th International Conference on Electrical Engineering, Computer Science and Informatics (EECSI)}

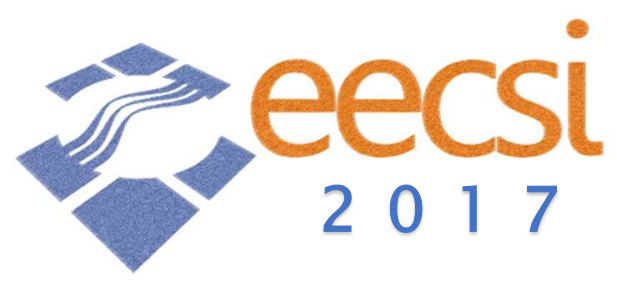

Copyright and Reprint Permission: Abstracting is permitted with credit to the source. Libraries are permitted to photocopy beyond the limit of U.S. copyright law for private use of patrons those articles in this volume that carry a code at the bottom of the first page, provided the per-copy fee indicated in the code is paid through Copyright Clearance Center, 222 Rosewood Drive, Danvers, MA 01923. For other copying, reprint or republication permission, write to IEEE Copyrights Manager, IEEE Operations Center, 445 Hoes Lane, Piscataway, NJ 08854. All rights reserved.

Copyright (C) 2017 by IEEE.

ISBN : 978-1-5386-0548-6 (PRINT, Part Number : CFP17B51-PRT)

ISBN : 978-1-5386-0547-9 (DVD Part Number : CFP17B51-CDR)

ISBN : 978-1-5386-0549-3 (XPLORE COMPLIANT, Part Number : CFP17B51-ART)

Additional copies may be ordered to:

Lembaga Penerbitan dan Publikasi Ilmiah (LPPI)

Universitas Ahmad Dahlan

Jl. Kapas 9, Semaki, Umbulharjo

Yogyakarta, Indonesia 55166 


\section{ORGANIZING COMMITTEE OF EECSI 2017 CONFERENCE}

\section{Steering Committee}

- Adam Skorek, IEEE MGA Awards and Recognation Chair (R7) Trois-Rivières, QC, Canada

- Satrio Dharmanto, IEEE Indonesia Section (Chair)

- Pekik Argo Dahono, IEEE Indonesia Chapters Chair (EdSoc/EDS/PELS/SPS)

- Mochamad Ashari, Telkom University, Bandung, Indonesia

- Tumiran, Universitas Gadjah Mada, Yogyakarta, Indonesia

- Hermawan, Universitas Diponegoro, Semarang, Indonesia

- Zainudin Nawawi, Universitas Sriwijaya, Palembang, Indonesia

- Rahmat Budiarto, Albaha University, Baha, Saudi Arabia

- Sri Arttini Dwi Prasetyowati, Universitas Islam Sultan Agung, Semarang, Indonesia

- Kartika Firdausy, Universitas Ahmad Dahlan, Yogyakarta, Indonesia

- Siti Nurmaini, Universitas Sriwijaya, Palembang, Indonesia

\section{General Chairs}

- Tole Sutikno, Universitas Ahmad Dahlan, Yogyakarta, Indonesia

- Suharyanto, Universitas Gadjah Mada, Yogyakarta, Indonesia

- Deris Stiawan, Universitas Sriwijaya, Palembang, Indonesia

\section{Finance and Treasurer}

- Wiwiek Fatmawati, Universitas Islam Sultan Agung, Semarang, Indonesia

- Lina Handayani, Universitas Ahmad Dahlan, Yogyakarta, Indonesia

\section{Publicity}

- Son Ali Akbar, Universitas Ahmad Dahlan, Yogyakarta, Indonesia

- Muhammad Qomaruddin, Universitas Islam Sultan Agung, Semarang, Indonesia

- Sam F Chaerul, Universitas Islam Sultan Agung, Semarang, Indonesia

- Ahmad Heryanto, Universitas Sriwijaya, Palembang, Indonesia

\section{General co-Chairs}

- I Wayan Mustika, IEEE Indonesia Section

- Imam Much Ibnu Subroto, Universitas Islam Sultan Agung, Semarang, Indonesia

- Zulfatman, Universitas Muhammadiyah Malang, Malang, Indonesia

- Krisna Adiyarta, Universitas Budi Luhur, Jakarta, Indonesia

\section{Publication}

- Balza Achmad, Universitas Gadjah Mada, Yogyakarta, Indonesia

- Mochammad Facta, Universitas Diponegoro, Semarang, Indonesia

\section{Public Relations}

- Aina Musdholifah, Universitas Gadjah Mada, Yogyakarta, Indonesia

- Arief Marwanto, Universitas Islam Sultan Agung, Semarang, Indonesia

- Muhammad Syafrullah, Universitas Budi Luhur, Jakarta, Indonesia

- Riky Dwi Puriyanto, Universitas Ahmad Dahlan, Yogyakarta, Indonesia 


\section{Technical Program Committee}

\section{Technical Program Committee}

\begin{tabular}{|c|c|c|}
\hline Rajan A & Tata Consultancy Services & India \\
\hline Ali Abbas & $\begin{array}{l}\text { HIAST Higher Institute for Applied Science and } \\
\text { Technology }\end{array}$ & Syria \\
\hline Hany Abd El-Aal & Cairo University & Egypt \\
\hline Saied Abd El-atty & Menoufia University-Faculty of Electronic Engineering & Egypt \\
\hline Malaoui Abdessamad & Sultan Moulay Slimane University of Beni Mellal & Morocco \\
\hline Fairul Azhar Abdul Shukor & Universiti Teknikal Malaysia Melaka & Malaysia \\
\hline Balza Achmad & Universitas Gadjah Mada (Indonesia) & Indonesia \\
\hline Muhammad Ishtiaq Ahmad & Beijing Institute of Technology & P.R. China \\
\hline Hamid Alasadi & IRAQ- BASRA & Iraq \\
\hline Mohammed Alghamdi & Al-Baha University & Saudi Arabia \\
\hline Mehran Alidoost Nia & University of Tehran & Iran \\
\hline Gholamreza Alirezaei & RWTH Aachen University & Germany \\
\hline Yeasir Arafat & Bangladesh University of Engineering and Technology & Bangladesh \\
\hline Diego Arcos-Aviles & Universidad de las Fuerzas Armadas ESPE & Ecuador \\
\hline Ramy Atawia & Queen's University & Canada \\
\hline Shakti Awaghad & GHRCE, Nagpur & India \\
\hline Eduard Babulak & Fort Hays State University & USA \\
\hline Peter Balazs & Austrian Academy of Sciences & Austria \\
\hline Marco Baldi & Università Politecnica delle Marche & Italy \\
\hline $\begin{array}{l}\text { Herath Mudiyanselage Nelanga } \\
\text { Bandara }\end{array}$ & University of Moratuwa & Sri Lanka \\
\hline Ihsen Ben Mbarek & National Engineering School of Tunis & Tunisia \\
\hline Alper Bereketli & ASELSAN Inc. & Turkey \\
\hline Tuğçe Bilen & Istanbul Technical University & Turkey \\
\hline Rodrigo Campos Bortoletto & $\begin{array}{l}\text { São Paulo Federal Institute of Education, Science and } \\
\text { Technology }\end{array}$ & Brazil \\
\hline Yue Cao & Northumbria University & $\begin{array}{l}\text { United Kingdom (Great } \\
\text { Britain) }\end{array}$ \\
\hline Maria Chiara Caschera & CNR & Italy \\
\hline Arcangelo Castiglione & University of Salerno & Italy \\
\hline Suryadip Chakraborty & Johnson C. Smith University & USA \\
\hline Parag Chatterjee & National Technological University, Buenos Aires & Argentina \\
\hline Di Chen & University of Rostock & Germany \\
\hline Adilson Chinatto & Espectro Ltd. & Brazil \\
\hline Salim Chitroub & Electronics and Computer Science Faculty, USTHB & Algeria \\
\hline B Chitti Babu & The University of Nottingham Malaysia Campus & Malaysia \\
\hline Domenico Ciuonzo & $\begin{array}{l}\text { Network Measurement and Monitoring (NM2), Naples, } \\
\text { IT }\end{array}$ & Italy \\
\hline Paolo Crippa & Università Politecnica delle Marche & Italy \\
\hline Sanjoy Debbarma & National Institute of Technology Meghalaya & India \\
\hline George Dekoulis & Aerospace Engineering Institute & Cyprus \\
\hline July Katherine Díaz Barriga & Universidad Distrital Francisco José de Caldas & Colombia \\
\hline Saurabh Dixit & Babu Banarsi Das University, Lucknow & India \\
\hline Noha El-Ganainy & Arab Academy of Science and Technology AAST & Egypt \\
\hline Mochammad Facta & Diponegoro University & Indonesia \\
\hline Wei Feng & Tsinghua University & P.R. China \\
\hline Andrea Fiaschetti & University of Rome "La Sapienza" & Italy \\
\hline Muftah Fraifer & IDC-CSIS-UL & Ireland \\
\hline Felix J. Garcia Clemente & University of Murcia & Spain \\
\hline Hamza Ghandorh & University of Western Ontario & Canada \\
\hline Wajeb Gharibi & Jazan University, KSA & Saudi Arabia \\
\hline Srideep Ghosh & ELTRON Wireless & India \\
\hline Syed Ameer Ahmed Gillani & Zhejiang University & P.R. China \\
\hline
\end{tabular}


Visvasuresh Victor

Govindaswamy

Lacrimioara Grama

Henry Griffith

Burhan Gulbahar

Berkin Güler

Rohit Gupta

Zulfatman Has

Meisam Hashemi

Sherief Hashima

Jun $\mathrm{He}$

Ahmed Helmy

A. K. M. Mahtab Hossain

Zhaozheng $\mathrm{Hu}$

Tilokchan Irengbam

Md. Moidul Islam

Anish Jindal

Mohammed Kaabar

Murali Krishna Kadiyala

Dimitrios Kallergis

Jyotirmoy Karjee

Inderpreet Kaur

Shanu Khan

Yassine Khlifi

Jens Klare

Bo Kong

Fukuro Koshiji

Deepika Koundal

Sunil Kumar

Takashi Kurimoto

Chia-Hung Lai

Jia-Han Li

Xiangguo Li

Xiaojun Li

David Luengo

Shahid Manzoor

Rajeev Mathur

Sukadev Meher

Ahmed Mobashsher

Ratan Kumar Mondal

Rodrigo Montufar-Chaveznava

Martin Mudroch

Bodhibrata Mukhopadhyay

Ronald Mulinde

Fernando Mussoi

Nagendra Kumar Nainar

Farid Naït-Abdesselam

Abdellah Najid

Amir Nakib

Syed Mohsen Naqvi

Joanna Isabelle Olszewska

Feng Ouyang

Michel Owayjan

Oskars Ozolins
Concordia University

USA

Technical University of Cluj-Napoca

Michigan State University

Ozyegin University

Koc University

Thapar University

University of Muhammadiyah Malang

University of Isfahan

Engineering dept, Nuclear Research Center, EAEA,

Cairo

University of New Brunswick

Qualcomm Technologies Inc.

University of Greenwich

Georgia Institute of Technology

Manipur University

Friedrich Schiller University Jena

Thapar University, Patiala (India)

Washington State University

Cisco Systems. Inc

University of Piraeus

Tata Consultancy Services

Chandigarh University, Mohali

AICTE

Umm Al-Qura University, KSA

Fraunhofer FHR

PLA University of Science and Technology

Tokyo Polytechnic University

Chitkara University

The LNM Institute of Information Technology, Jaipur

NII

National Cheng Kung University

National Taiwan University

Henan University of Technology

Texas A\&M University

Universidad Politecnica de Madrid (UPM)

UCSI Universiti Kuala Lumpur

Geetanjali Instt of Tech Studies, Udaipur

National Institute of Technology, Rourkela

The University of Queensland

Queensland University of Technology

Facultad de Ingeniería, Universidad Nacional

Autonoma de Mexico

Czech Technical University in Prague

Indian Institute of Technology Delhi

University of South Australia

Federal Institute of Santa Catarina

CISCO

Paris Descartes University

Institut National des Postes et Télécommunications

University Paris East

Newcastle University

University of Gloucestershire

Johns Hopkins University / Applied Physics Lab

American University of Science \& Technology

RISE Acreo
Romania

USA

Turkey

Turkey

India

Indonesia

Iran

Egypt

Canada

USA

United Kingdom (Great

Britain)

USA

India

Germany

India

USA

USA

Greece

India

India

India

Saudi Arabia

Germany

P.R. China

Japan

India

India

Japan

Taiwan

Taiwan

P.R. China

USA

Spain

Malaysia

India

India

Australia

Australia

Mexico

Czech Republic

India

Australia

Brazil

USA

France

Morocco

France

United Kingdom (Great

Britain)

United Kingdom (Great

Britain)

USA

Lebanon

Sweden 
Shashikant Patil

Shashikant Patil

Gabriele Piantadosi

Rajesh Pindoriya

Octavian Postolache

Adhi Prahara

Nadia Qasim

Harikumar Rajaguru

Shuvendu Rana

Hemant Kumar Rath

Muhammad Raza

Mohamed Rehan

Abdalhossein Rezai

Munawar Riyadi

Indra Riyanto

Olympia Roeva

Karla Maria Ronquillo Gonzalez

Muthukumar S

Zulhisyam Salleh

Aratã Saraiva

Gnane Swarnadh Satapathi

Hans Schotten

Ljiljana Šerić

Aditi Sharma

Satish Sharma

Rupesh Singh

Saurabh Srivastava

Deris Stiawan

Ravi Subban

Imam Much Ibnu Subroto

Hengky Susanto

TH Sutikno

Tole Sutikno

Srinivasulu Tadisetty

Pooya Taheri

Min Keng Tan

Xuanxuan Tang

Revak Tyagi

Sudhanshu Tyagi

Dimitris Varoutas

Marcel Wagner

Hao Wu

Kun-Da Wu

Kishore Yalamanchili

Mohammed Younis

Anton Yudhana

Pujianto Yugopuspito

Nemanja Zdravkovic

Chi Zhang

Zhe Zhang

Quanxin Zhao

Jing Zhou

Tudor Cătălin Zorilă
SVKMs NMiMS Mumbai India

SVKM NMIMS Mumbai India

University of Naples Federico II

Indian Institute of Technology Mandi

Instituto de Telecomunicações, Lisboa/IT

Universitas Ahmad Dahlan

King's College London

anna University Chennai

University of Strathclyde

Tata Consultancy Services

HUST Wuhan China

AvidBeam Technlogies

ACECR

Diponegoro University

Universitas Budi Luhur

Institute of Biophysics and Biomedical Engineering

Universidad Tecnológica de Chihuahua

Indian Institute of Information Technology, Tamilnadu

Politeknik Melaka

UESPI

National Institute of Technology, Karnataka

University of Kaiserslautern

University of Split - Faculty of El. Eng., Mech. Eng.

and Naval Arch.

MBM Engineering College Jodhpur

ISRO Satellite Centre

Tantia University

NIt $\mathrm{K}$

University of Sriwijaya

Pondicherry University, Pondicherry

Universitas Islam Sultan Agung

Huawei Technology

Institute of Advanced Engineering and Science

Universitas Ahmad Dahlan

Kakatiya University College of Engineering and

Technology

SFU

Universiti Malaysia Sabah

PLA Army Engineering University

Cisco Systems

Thapar University, Patiala

University of Athens

University of São Paulo

ZTE Corporation

HTC Corporation

Google

University of Baghdad

Ahmad Dahlan University

Universitas Pelita Harapan

Norwegian University of Science and Techonlogy

Midea America Corporation

Geroge Mason University

University of Electronic Science and Technology of

China

University of Science and Technology of China

Toshiba Cambridge Research Laboratory
India

India

Italy

India

Portugal

Indonesia

United Kingdom (Great

Britain)

India

United Kingdom (Great

Britain)

India

P.R. China

Egypt

Iran

Indonesia

Indonesia

Bulgaria

Mexico

India

Malaysia

Brazil

India

Germany

Croatia

India

India

India

India

Indonesia

India

Indonesia

Hong Kong

Indonesia

Indonesia

India

Canada

Malaysia

P.R. China

USA

India

Greece

Brazil

P.R. China

Taiwan

USA

Iraq

Indonesia

Indonesia

Norway

USA

USA

P.R. China

P.R. China

United Kingdom (Great

Britain) 


\section{Other reviewers}

\section{Additional Reviewers}

\begin{tabular}{|l|}
\hline Hany Ali Abd El-Aal \\
Malaoui Abdessamad \\
\hline Fairul Azhar Abdul Shukor \\
\hline Trio Adiono \\
\hline Andi Adriansyah \\
\hline Muhammad Ishtiaq Ahmad \\
\hline Son Akbar \\
\hline Hamid Alasadi \\
\hline Mudrik Alaydrus \\
\hline Mehran Alidoost Nia \\
\hline Fahad Alswaina \\
\hline Sahil Anchal \\
\hline Trias Andromeda \\
\hline Nor Badrul Anuar \\
\hline Khoirul Anwar \\
\hline Diego Arcos-Aviles \\
\hline Anu Shaju Areeckal \\
\hline Yanuar Arief \\
\hline Mochammad Ariyanto \\
\hline Eduard Babulak \\
\hline Basari Basari \\
\hline Alper Bereketli \\
\hline Tuğçe Bilen \\
\hline
\end{tabular}

Rodrigo Campos Bortoletto

Christos-Savvas Bouganis

Arif Bramantoro

Rahmat Budiarto

Feri Candra

Yue Cao

Alberto Carboni

Maria Chiara Caschera

Arcangelo Castiglione

Sam F Chaerul Haviana

Che Haziqah Che Hussin

Di Chen

Adilson W Chinatto, Jr.

Paolo Crippa

Esmeralda Contessa

Djamal

Drazen Dujic

Mohammed El Hassouni

Hala El Ouarrak

Noha Ossama El-Ganainy

Wei Feng

Muftah Fraifer

Sibaji Gaj

Felix J. Garcia Clemente

Hamza Ghandorh

Srideep Ghosh

Syed Ameer Ahmed Gillani

Lacrimioara Grama
Cairo University

Sultan Moulay Slimane University of Beni Mellal

Universiti Teknikal Malaysia Melaka

STEI ITB

Universitas Mercu Buana

Beijing Institute of Technology

Universitas Ahmad Dahlan

IRAQ- BASRA

Universitas Mercu Buana

University of Tehran

University of Bridgeport

Indian Institute of Technology Delhi

Universiti Teknologi Malaysia

University of Malaya

Telkom University

Universidad de las Fuerzas Armadas ESPE

National Institute of Technology Karnataka, Surathkal

UNIMAS

Diponegoro University

Fort Hays State University

Universitas Indonesia

ASELSAN Inc.

Istanbul Technical University

São Paulo Federal Institute of Education, Science and

Technology

Imperial College London

Al Imam Mohammad Ibn Saud Islamic University

Al Baha University

Universiti Teknologi Malaysia

Northumbria University

Politecnico di Milano

CNR

University of Salerno

Universitas Islam Sultan Agung

University Malaysia Sabah

University of Rostock

Espectro Ltd.

Università Politecnica delle Marche

Universitas Jenderal Achmad Yani

EPFL

University of Mohammed $\mathrm{V}$-Agdal-

University Hassan II Mohammedia

Arab Academy of Science and Technology AAST

Tsinghua University

IDC-CSIS-UL

IIT Guwahati

University of Murcia

University of Western Ontario

ELTRON Wireless

Zhejiang University

Technical University of Cluj-Napoca

\begin{tabular}{|l}
\hline Egypt \\
\hline Morocco \\
\hline Malaysia \\
\hline Indonesia \\
\hline Indonesia \\
\hline P.R. China \\
\hline Indonesia \\
\hline Iraq \\
\hline Indonesia \\
\hline Iran \\
\hline USA \\
\hline India \\
\hline Malaysia \\
\hline Malaysia \\
\hline Indonesia \\
\hline Ecuador \\
\hline India \\
\hline Malaysia \\
\hline Indonesia \\
\hline USA \\
\hline Indonesia \\
\hline Turkey \\
\hline Turkey \\
\hline Brazil \\
\hline United Kingdom (Great \\
\hline Britain) \\
\hline Saudi Arabia \\
\hline Saudi Arabia \\
\hline Malaysia \\
\hline United Kingdom (Great \\
Britain) \\
\hline Italy \\
\hline Italy \\
\hline Italy \\
\hline Indonesia \\
\hline Malaysia \\
\hline Germany \\
\hline Rrazia \\
\hline Italy \\
\hline Indonesia \\
\hline Swaziland \\
\hline Morocco \\
\hline Morocco \\
\hline Egypt \\
\hline In. China \\
\hline
\end{tabular}


Henry Griffith

Berkin Güler

Teddy Surya Gunawan

Rohit Gupta

Bagus Haryadi

Zainal Hasibuan

Abdolreza Hatamlou

Jun $\mathrm{He}$

Ahmad Heryanto

Tris Dewi Indraswati

Tilokchan Irengbam

Nanang Ismail

Rifky Ismail

R Rizal Isnanto

Anish Jindal

Murali Krishna Kadiyala

Dimitrios Kallergis

Moh Khairudin Khairudin,

$\mathrm{Mr}$

Umar Khayam

Yassine Khlifi

Jens Klare

Bo Kong

Deepika Koundal

Takashi Kurimoto

Fajri Kurniawan

Xiangguo Li

Xiaojun Li

David Luengo

Satria Mandala

Muhammad Nadzir

Marsono

Arief Marwanto

Rajeev Mathur

Amir Hooshang Mazinan

Siti Armiza Mohd Aris

Martin Mudroch

Bodhibrata Mukhopadhyay

Prasetiyono Hari Mukti

Ronald Mulinde

Indra $\mathrm{H}$ Mulyadi

Achmad Munir

Aina Musdholifah

Fernando L. R. Mussoi

Imamul Muttakin

Imamul Muttakin

Adharul Muttaqin

Abdellah Najid

Syed Mohsen Naqvi

Oky Nurhayati

Joanna Isabelle Olszewska

Oskars Ozolins

Shashikant Shantilal Patil

Gabriele Piantadosi

Rajesh M Pindoriya

Octavian Adrian

Postolache
Michigan State University

Koc University

International Islamic University Malaysia

Thapar University

Universitas Ahmad Dahlan

University of Indonesia

Islamic Azad University, Khoy Branch

University of New Brunswick

Sriwijaya University

Institut Teknologi Indonesia

Manipur University

UIN Bandung

Diponegoro University

Diponegoro University

Thapar University, Patiala (India)

Cisco Systems. Inc

University of Piraeus

Universitas Negeri Yogyakarta

Institut Teknologi Bandung

Umm Al-Qura University, KSA

Fraunhofer FHR

PLA University of Science and Technology

Chitkara University

NII

Universiti Teknologi Malaysia

Henan University of Technology

Texas A\&M University

Universidad Politecnica de Madrid (UPM)

Universitas Telkom

Universiti Teknologi Malaysia

Universiti Islam Sultan Agung (UNISSULA) Semarang

Geetanjali Instt of Tech Studies, Udaipur

Islamic Azad Unversity, South Tehran Branch

Universiti Teknologi Malaysia

Czech Technical University in Prague

Indian Institute of Technology Delhi

Institut Teknologi Sepuluh Nopermber

University of South Australia

Technische Universität IImenau

Institut Teknologi Bandung

Gadjah Mada University

Federal Institute of Santa Catarina

CTECH Labs Edwar Technology Co.

Universitas Sultan Ageng Tirtayasa

Universitas Brawijaya

Institut National des Postes et Télécommunications

Newcastle University

Diponegoro University

University of Gloucestershire

RISE Acreo

SVKM NMIMS Mumbai India

University of Naples Federico II

Indian Institute of Technology Mandi

Instituto de Telecomunicações, Lisboa/IT
USA

Turkey

Malaysia

India

Indonesia

Indonesia

Iran

Canada

Indonesia

Indonesia

India

Indonesia

Indonesia

Indonesia

India

USA

Greece

Indonesia

Indonesia

Saudi Arabia

Germany

P.R. China

India

Japan

Malaysia

P.R. China

USA

Spain

Indonesia

Malaysia

Indonesia

India

Iran

Malaysia

Czech Republic

India

Indonesia

Australia

Germany

Indonesia

Indonesia

Brazil

Indonesia

Indonesia

Indonesia

Morocco

United Kingdom (Great

Britain)

Indonesia

United Kingdom (Great

Britain)

Sweden

India

Italy

India

Portugal 
Teguh Prakoso

Agus Pratondo

Gigih Priyandoko

Nadia Qasim

Muhammad Qomaruddin

Tri Desmana Rachmilda

Wenny Rahayu

Basuki Rahmat

Harikumar Rajaguru

Kalamullah Ramli

Shuvendu Rana

Muhammad Raza

Indra Riyanto

Olympia Roeva

Zulhisyam Salleh

Gnane Swarnadh

Satapathi, Er

Haikal Satria

Dian Sawitri

Hans D. Schotten

Stefan Schuster

Ali Selamat

Norhalina Senan

Noor Akhmad Setiawan

Aditi Sharma

Edelberto Franco Silva

Aghus Sofwan

Maman Somantri

Saurabh Srivastava

Ravi Subban

Rubita Sudirman

Sunardi Sunardi

Iping Supriana

Fajar Suryawan

Muhammad Syafrullah

Abdul Syakur

Srinivasulu Tadisetty

Xuanxuan Tang

Fritzi Töpfer

Revak R Tyagi

Sudhanshu Tyagi

Marcel Stefan Wagner

Jing Wang

Setyawan Widyarto

Augie Widyotriatmo

Hao Wu

Mohammed I. Younis

Pujianto Yugopuspito

Arda Yunianta

Eniman Yunus

Chi Zhang

Zhe Zhang

Jing Zhou

Tudor Cătălin Zorilă

Sonny Zulhuda
Diponegoro University

NUS

UMP

King's College London

Universitas Islam Sultan Agung (UNISSULA)

Institut Teknologi Bandung

La Trobe University

Telkom University

anna University Chennai

Universitas Indonesia

University of Strathclyde

HUST Wuhan China

Universitas Budi Luhur

Institute of Biophysics and Biomedical Engineering

Politeknik Melaka

National Institute of Technology, Karnataka

Universiti Teknologi Malaysia

UDINUS

University of Kaiserslautern

Voestalpine Stahl Gmbh

Universiti Teknologi Malaysia

UTHM

Universitas Gadjah Mada

MBM Engineering College Jodhpur

Universidade Federal de Juiz de Fora

Diponegoro University

Gadjah Mada University

NIt K

Pondicherry University, Pondicherry

Universiti Teknologi Malaysia

Universitas Ahmad Dahlan

Bandung Institute of Technology

Universitas Muhammadiyah Surakarta

Universitas Budi Luhur

Diponegoro University

Kakatiya University College of Engineering and Technology

PLA Army Engineering University

KTH Royal Institute of Technology

Cisco Systems

Thapar University, Patiala

University of São Paulo

Georgia Institute of Technology

Universiti Selangor

Institut Teknologi Bandung

ZTE Corporation

University of Baghdad

Universitas Pelita Harapan

King Abdulaziz University

Institut Teknologi Bandung

Midea America Corporation

Geroge Mason University

University of Science and Technology of China

Toshiba Cambridge Research Laboratory

International Islamic University Malaysia
Indonesia

Singapore

Malaysia

United Kingdom (Great

Britain)

Indonesia

Indonesia

Australia

Indonesia

India

Indonesia

United Kingdom (Great

Britain)

P.R. China

Indonesia

Bulgaria

Malaysia

India

Malaysia

Indonesia

Germany

Austria

Malaysia

Malaysia

Indonesia

India

Brazil

Indonesia

Indonesia

India

India

Malaysia

Indonesia

Indonesia

Indonesia

Indonesia

Indonesia

India

P.R. China

Sweden

USA

India

Brazil

USA

Malaysia

Indonesia

P.R. China

Iraq

Indonesia

Saudi Arabia

Indonesia

USA

USA

P.R. China

United Kingdom (Great

Britain)

Malaysia 


\section{TABLE OF CONTENTS}

\begin{tabular}{|c|c|}
\hline $\begin{array}{l}\text { High Performance Direct Torque Control of Induction Motor Drives: } \\
\text { Problems and Improvements } \\
\text { Nik Rumzi Nik Idris (UTM-PROTON Future Drive Laboratory, Universiti } \\
\text { Teknologi Malaysia, Johor, Malaysia), Tole Sutikno (Department of Electrical } \\
\text { Engineering, Universitas Ahmad Dahlan, Yogyakarta, Indonesia) }\end{array}$ & 1 \\
\hline $\begin{array}{l}\text { Towards Development of A Computerised System for Screening and } \\
\text { Monitoring of Diabetic Retinopathy } \\
\text { Hanung Adi Nugroho (Universitas Gadjah Mada, Indonesia) }\end{array}$ & 8 \\
\hline $\begin{array}{l}\text { Performance Analysis of Network Emulator Based On The Use Of } \\
\text { Resources In Virtual Laboratory } \\
\text { Yuri Ariyanto, Yan Watequlis Syaifudin, Budi Harijanto (State Polytechnic of } \\
\text { Malang,Malang, East Java, Indonesia) }\end{array}$ & 9 \\
\hline $\begin{array}{l}\text { Teaching And Learning Support For Computer Architecture And } \\
\text { Organization Courses Design On Computer Engineering and Computer } \\
\text { Science For Undergraduate: A Review } \\
\text { Wijaya Kurniawan,Mochammad Hannats Hanafi Ichsan (Brawijaya University, } \\
\text { Malang, East Java, Indonesia) }\end{array}$ & 15 \\
\hline $\begin{array}{l}\text { WatsaQ: Repository of Al Hadith in Bahasa (Case Study: Hadith Bukhari) } \\
\text { Atqia Aulia, Dewi Khairani, Rizal Broer Bahaweres, and Nashrul Hakiem } \\
\text { (Department of Informatics, UIN Syarif Hidayatullah, Jakarta, Indonesia) }\end{array}$ & 21 \\
\hline $\begin{array}{l}\text { IoT Smart Device for e-Learning Content Sharing on Hybrid Cloud } \\
\text { Environment } \\
\text { Mohd. Yazid Idris, Deris Stiawan, Nik Mohd Habibullah, Abdul Hadi Fikri, Mohd } \\
\text { Rozaini Abd Rahim, Massolehin Dasuki (Universiti Teknologi Malaysia, Johor } \\
\text { Bahru, Malaysia) }\end{array}$ & 25 \\
\hline $\begin{array}{l}\text { Target Tracking in Mobile Robot under Uncertain Environment using } \\
\text { Fuzzy Logic Controller } \\
\text { Ade Silvia Handayani, Tresna Dewi, Nyayu Latifah Husni (State Polytechnic of } \\
\text { Sriwijaya,Palembang, Indonesia), Siti Nurmaini, Irsyadi Yani (University of } \\
\text { Sriwijaya, Palembang, Indonesia) }\end{array}$ & 30 \\
\hline $\begin{array}{l}\text { Nitrogen (N) Fertilizer Measuring Instrument On Maize-Based Plant } \\
\text { Microcontroller } \\
\text { Hendra Yufit Riskiawan, Taufiq Rizaldi, Dwi Putro S. Setyohadi, Tri Leksono } \\
\text { (Information Technology Department, Politeknik Negeri Jember, Indonesia) }\end{array}$ & 35 \\
\hline $\begin{array}{l}\text { Reconfigurable Logic Embedded Architecture of Support Vector Machine } \\
\text { Linear Kernel } \\
\text { Jeevan Sirkunan, N. Shaikh-Husin and M. N. Marsono (Fac. of Electrical Eng., } \\
\text { Universiti Teknologi Malaysia, Johor, Malaysia), Trias Andromeda (Diponegoro } \\
\text { University, Semarang, Indonesia) }\end{array}$ & 39 \\
\hline
\end{tabular}




\begin{tabular}{|c|c|}
\hline $\begin{array}{l}\text { An Analysis of Concentration Region on Powerpoint Slides using Eye } \\
\text { Tracking } \\
\text { Fergyanto E. Gunawan, Oky Wijaya, Benfano Soewito, Sevenpri Candra Diana } \\
\text { (Bina Nusantara University, Jakarta, Indonesia) Cosmas E. Suharyanto( Putera } \\
\text { Batam University, Riau Archipelago, Indonesia) }\end{array}$ & 44 \\
\hline $\begin{array}{l}\text { Implementation of K-Means Clustering Method to Distribution of High } \\
\text { School Teachers } \\
\text { Triyanna Widiyaningtyas, Martin Indra Wisnu Prabowo, M. Ardhika Mulya } \\
\text { Pratama (Electrical Engineering Departement, Universitas Negeri Malang, } \\
\text { Malang, Indonesia) }\end{array}$ & 49 \\
\hline $\begin{array}{l}\text { Incremental High Throughput Network Traffic Classifier } \\
\text { H. R. Loo, Alireza Monemi, and M. N. Marsono (Faculty of Electrical } \\
\text { Engineering, Universiti Teknologi Malaysia, Johor, Malaysia), Trias Andromeda } \\
\text { ( Diponegoro University, Semarang, Indonesia) }\end{array}$ & 55 \\
\hline $\begin{array}{l}\text { Edge Detection on Objects of Medical Image with Enhancement multiple } \\
\text { Morphological Gradient Method } \\
\text { Jufriadif Na`am (Computer Science Faculty, Universitas Putra Indonesia YPTK, } \\
\text { Padang, Indonesia) }\end{array}$ & 61 \\
\hline $\begin{array}{l}\text { Unified Concept-based Multimedia Information Retrieval Technique } \\
\text { Ridwan Andi Kambau, Zainal Arifin Hasibuan (Faculty of Computer Science, } \\
\text { University of Indonesia, Depok, West Java, Indonesia) }\end{array}$ & 68 \\
\hline $\begin{array}{l}\text { Text Modeling In Adaptive Educational Chat Room Based On Madamira } \\
\text { Tool } \\
\text { Jehad A. H. Hammad, Mochamad Hariadi, Mauridhi Hery Purnomo } \\
\text { (Department of Computer Engineering, Institut Teknologi Sepuluh Nopember } \\
\text { (ITS) Surabaya, Indonesia), Nidal A. M Jabari (Department of Computer, } \\
\text { Technical Colleges(Arroub), Palestine) }\end{array}$ & 76 \\
\hline $\begin{array}{l}\text { Analysis of Statement Branch and Loop Coverage in Software Testing } \\
\text { With Genetic Algorithm } \\
\text { Rizal Broer Bahaweres }{ }^{1,2} \text {, Khoirunnisya Zawawi }{ }^{1} \text {, Dewi Khairani }{ }^{1} \text {, Nashrul } \\
\text { Hakiem }{ }^{1}\left({ }^{1} \text { Department of Informatics, Syarif Hidayatullah State Islamic }\right. \\
\text { University, Jakarta, Indonesia }{ }^{2} \text { Faculty of Computer Science, NRU Higher } \\
\text { School of Economics, Moscow, Russia ) }\end{array}$ & 82 \\
\hline 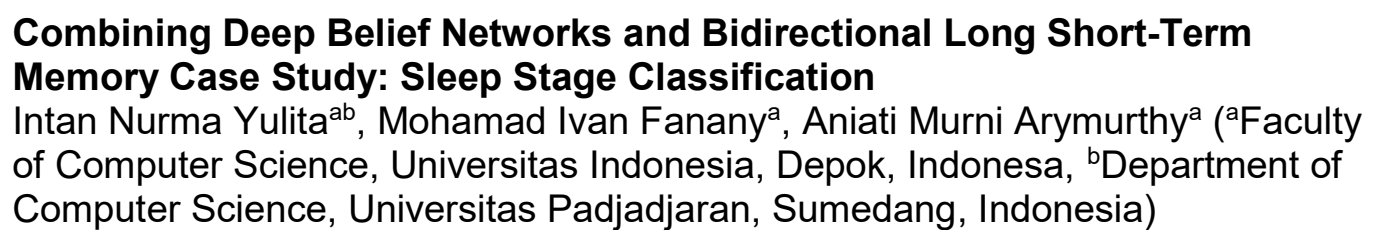 & 88 \\
\hline $\begin{array}{l}\text { Improvement of eGov \& mGov in Multilingual Countries with Digital } \\
\text { Etymology using Sanskrit Grammar } \\
\text { Arijit Das }\end{array}$ & 91 \\
\hline
\end{tabular}




\begin{tabular}{|c|c|}
\hline $\begin{array}{l}\text { EEG Based Emotion Monitoring Using Wavelet and Learning Vector } \\
\text { Quantization } \\
\text { Esmeralda C. Djamal and Poppi Lodaya (Universitas Jenderal Achmad Yani, } \\
\text { Bandung, Indonesia) }\end{array}$ & 94 \\
\hline $\begin{array}{l}\text { Myoelectric control systems for hand rehabilitation device: a review } \\
\text { Khairul Anam, Ahmad Adib Rosyadi, Bambang Sujanarko (University of } \\
\text { Jember, Jember, Indonesia), Adel Al-Jumaily (School of Biomedical } \\
\text { Engineering, University of Technology, Sydney, Australia) }\end{array}$ & 100 \\
\hline $\begin{array}{l}\text { Variance Analysis of Photoplethysmography for Blood Pressure } \\
\text { Measurement } \\
\text { Hendrana Tjahjadi, Kalamullah Ramli (Departement of Electrical Engineering, } \\
\text { Universitas Indonesia, Depok, Indonesia) }\end{array}$ & 106 \\
\hline $\begin{array}{l}\text { Implementation of Unbiased Stereology Method for Organ Volume } \\
\text { Estimation using Image Processing }\end{array}$ & 110 \\
\hline $\begin{array}{l}\text { Mohammad Ammar Faiq, Balza Achmad, Ginus Partadiredja (Universitas Gadjah } \\
\text { Mada Yogyakarta, Indonesia) }\end{array}$ & \\
\hline $\begin{array}{l}\text { Ethnobotany Database: Exploring diversity medicinal plants of Dayak } \\
\text { Tribe Borneo } \\
\text { Haeruddin }{ }^{1} \text {, Ummul Hairah }{ }^{1} \text {, Edy Budiman }{ }^{1} \text {, Herni Johan (Department of } \\
\text { Computer Science and Information Technology, Universitas Mulawarman } \\
\text { Samarinda - Indonesia, }{ }^{2} \text { Departement of Mutiara Mahakam Academy of } \\
\text { Midwifery, AKBID Samarinda, Samarinda - Indonesia) }\end{array}$ & 116 \\
\hline $\begin{array}{l}\text { Automated Post-Trabeculectomy Bleb Assesment by Using Image } \\
\text { Processing }\end{array}$ & 122 \\
\hline $\begin{array}{l}\text { Agwin Fahmi Fahanani, Hasballah Zakaria, Andika Prahasta, Elsa Gustianty, R. } \\
\text { Maula Rifada, Astrid Chairini (Department of Biomedical Engineering, Institut } \\
\text { Teknologi Bandung, Bandung, Indonesia) }\end{array}$ & \\
\hline Non-invasive Hemoglobin Measurement for Anemia Diagnosis & 125 \\
\hline $\begin{array}{l}\text { Raditya Artha Rochmanto, Hasballah Zakaria, Ratih Devi Alviana, Nurhalim } \\
\text { Shahib ('Department of Biomedical Engineering, Institut Teknologi Bandung, } \\
{ }^{2} \text { Medical Faculty Padjajaran University Bandung, Indonesia Bandung, } \\
\text { Indonesia) }\end{array}$ & \\
\hline $\begin{array}{l}\text { Poincaré plot of fingertip photoplethysmogram pulse amplitude suitable } \\
\text { to assess diabetes status }\end{array}$ & 130 \\
\hline $\begin{array}{l}\text { Bagus Haryadi }{ }^{1,2} \text {, Lin, Gen-Min }{ }^{2} ; \text { Yang, Chieh-Ming }{ }^{2} \text {; Chu, Shiao- Chiang }{ }^{2} ; \text { Wu, } \\
\text { Hsien-Tsai ( Department of Electrical Engineering National Dong-Hwa } \\
\text { University Hualien, Taiwan) }\end{array}$ & \\
\hline $\begin{array}{l}\text { Certain Factor Analysis for Extra Pulmonary Tuberculosis Diagnosis } \\
\text { Ramadiani, Nur Aini, Heliza Rahmania Hatta, Fahrul Agus, Zainal Ariffin, Azainil } \\
\text { (Mulawarman University, Samarinda, Indonesia) }\end{array}$ & 134 \\
\hline
\end{tabular}




\begin{tabular}{|c|c|}
\hline $\begin{array}{l}\text { The Improvement of Phonocardiograph Signal (PCG) Representation } \\
\text { Through the Electronic Stethoscope } \\
\text { Sumarna, Juli Astono, Agus Purwanto, Dyah Kurniawati Agustika (Universitas } \\
\text { Negeri Yogyakarta) }\end{array}$ & 141 \\
\hline $\begin{array}{l}\text { Neural Network on Mortality Prediction for the Patient Admitted } \\
\text { with ADHF (Acute Decompensated Heart Failure) } \\
\text { M. Haider Abu Yazid, Shukor Talib, Muhammad Haikal Satria (Universiti } \\
\text { Teknologi Malaysia (UTM), Johor Bahru, Malaysia) Azmee Abd Ghazi (National } \\
\text { Heart Institute (IJN), Kuala Lumpur, Malaysia) }\end{array}$ & 146 \\
\hline $\begin{array}{l}\text { Measurement Of Maximum Value Of Dental Radiograph To Predict The } \\
\text { Bone Mineral Density }\end{array}$ & 152 \\
\hline $\begin{array}{l}\text { Sri Lestari, Mohammad Diqi (Faculty of Science and Technology, UNRIYO } \\
\text { Yogyakarta, Indonesia), Rini Widyaningrum (Departement of Dentomaxillofacial } \\
\text { Radiology Faculty of Dentistry, Universitas Gadjah Mada Yogyakarta, } \\
\text { Indonesia) }\end{array}$ & \\
\hline $\begin{array}{l}\text { Feature Extraction and Classification of Thorax X-Ray Image in the } \\
\text { Assessment of Osteoporosis }\end{array}$ & 156 \\
\hline $\begin{array}{l}\text { Riandini, Mera Kartika Delimayanti (Politeknik Negeri Jakarta Kampus UI } \\
\text { Depok West Java) }\end{array}$ & \\
\hline $\begin{array}{l}\text { 2D-Sigmoid Enhancement Prior to Segment MRI Glioma Tumour Pre } \\
\text { Image-Processing }\end{array}$ & 161 \\
\hline $\begin{array}{l}\text { Setyawan Widyarto, Siti Rafidah Binti Kassim (Faculty of Communication, } \\
\text { Visual Art and Computing, UNISEL, Kuala Selangor, Malaysia), Widya Kumala } \\
\text { Sari (Alumni of Faculty of Medicine, Universitas Gadjah Mada Indonesia) }\end{array}$ & \\
\hline $\begin{array}{l}\text { Alerting System for Sport Activity Based on ECG Signals using } \\
\text { Proportional Integral Derivative }\end{array}$ & 166 \\
\hline $\begin{array}{l}\text { Vika Octaviani, Arief Kurniawan, Yoyon Kusnendar Suprapto, Ahmad Zaini } \\
\text { (Institut Teknologi Sepuluh Nopember, Surabaya Indonesia) }\end{array}$ & \\
\hline $\begin{array}{l}\text { Design of Automatic Switching Bio-Impedance Analysis (BIA) for Body Fat } \\
\text { Measurement }\end{array}$ & 172 \\
\hline $\begin{array}{l}\text { Munawar A Riyadi, Achmad Ngaqib Muthouwali, Teguh Prakoso (Department } \\
\text { of Electrical Engineering, Diponegoro University, Semarang, Indonesia) }\end{array}$ & \\
\hline $\begin{array}{l}\text { Precise Wide Baseline Stereo Image Matching for Compact Digital } \\
\text { Cameras } \\
\text { Martinus Edwin Tjahjadi, Fourry Handoko (National Institute of Technology } \\
\text { (ITN) Malang, Malang, Indonesia) }\end{array}$ & 177 \\
\hline $\begin{array}{l}\text { Robust and Imperceptible Image Watermarking by DC Coefficients Using } \\
\text { Singular Value Decomposition }\end{array}$ & 183 \\
\hline
\end{tabular}


Christy Atika Sari, Eko Hari Rachmawanto, De Rosal Ignatius Moses Setiadi

(Dian Nuswantoro University (UDINUS), Semarang, Indonesia)

Region of Interest Detection for Pregnancy Image Processing

M. Khairudin, Joko Laras B T, Dessy Irmawati (Universitas Negeri Yogyakarta, Yogyakarta,Indonesia)

Shape Defect Detection for Product Quality Inspection and monitoring System

Norhashimah Mohd Saad ${ }^{1}$, Nor Nabilah Syazana Abdul Rahman ${ }^{1}$, Abdul Rahim Abdullah( Universiti Teknikal Malaysia Melaka, Durian Tunggal, Melaka), Farhan Abdul Wahab (Infineon Technologies Sdn. Bhd, Batu Berendam, Melaka)

Toward a New Approach in Fruit Recognition using Hybrid RGBD Features and Fruit Hierarchy Property

Ema Rachmawati, Iping Supriana, Masayu Leylia Khodra (School of Electrical Engineering and Informatics, Institut Teknologi Bandung)

Mobile Content Based Image Retrieval Architectures

Arif Rahman (Universitas Ahmad Dahlan), Edi Winarko, Moh. Edi Wibowo (Universitas Gadjah Mada, Yogyakarta, Indonesia)

Computer Vision Based Object Tracking as a Teaching Aid for High School Physics Experiments

G.D. Illeperuma (The Open University of Sri Lanka, Nawala, Nugegoda, Sri Lanka), D.U.J. Sonnadara(University of Colombo, Sri Lanka)

Texture Analysis and Fracture Identification of Lower Extremity Bones XRay Images

Rahayu Suci Prihatini, Anif Hanifa Setyaningrum, Imam Marzuki Shofi (Departement of Informatics Engineering, UIN Syarif Hidayatullah, Jakarta, Indonesia)

Analysis of the Indonesian Vowel /e/ For Lip Synchronization Animation Anung Rachman, Risanuri Hidayat, Hanung Adi Nugroho (Universitas Gadjah Mada, Yogyakarta, Indonesia)

\section{Anti-Cheating Presence System Based on 3WPCA- Dual Vision Face} Recognition

Edy Winarno, Wiwien Hadikurniawati, Imam Husni Al Amin, Muji Sukur (Faculty of Information Technology, Universitas Stikubank Semarang Indonesia)

Sketch Plus Colorization Deep Convolutional Neural Networks for Photos Generation from Sketches

Vinnia Kemala Putri and Mohamad Ivan Fanany (Faculty of Computer Science, Universitas Indonesia, Depok, West-Java Indonesia)

Imperceptible Image Watermarking based on Chinese Remainder Theorem over the Edges 


\begin{tabular}{|c|c|}
\hline $\begin{array}{l}\text { Informatics Engineering Universitas Dian Nuswantoro (UDINUS) Semarang, } \\
\text { Indonesia) }\end{array}$ & \\
\hline $\begin{array}{l}\text { Wood Texture Detection with Conjugate Gradient Neural Network } \\
\text { Algorithm } \\
\text { Setyawan Widyarto, I Nyoman Suryasa, Otto Fajarianto (Universitas Budi } \\
\text { Luhur, Jakarta, Indonesia), Mohd Shafry Mohd Rahim (Universiti Teknologi } \\
\text { Malaysia, Johor Bahru, Malaysia), Khairul Annuar bin Abdullah (Universiti } \\
\text { Selangor, Malaysia), Gigih Priyandoko, Gilang Anggit Budaya (Universiti } \\
\text { Malaysia Pahang, Malaysia) }\end{array}$ & 240 \\
\hline $\begin{array}{l}\text { Spoken Word Recognition Using MFCC and Learning Vector Quantization } \\
\text { Esmeralda C. Djamal, Neneng Nurhamidah and Ridwan Ilyas (Universitas } \\
\text { Jenderal Achmad Yani, Bandung, Indonesia) }\end{array}$ & 246 \\
\hline $\begin{array}{l}\text { A Hierarchical Description-based Video Monitoring System for Elderly } \\
\text { Mochamad Irwan Nari, Agung Wahyu Setiawan and Widyawardana Adiprawita } \\
\text { (Institut Teknologi Bandung, Indonesia) }\end{array}$ & 252 \\
\hline $\begin{array}{l}\text { Performance Measurement Based on Coloured Petri Net Simulation of } \\
\text { Scalable Business Processes } \\
\text { Abd. Charis Fauzan, Riyanarto Sarno, Muhammad Ainul Yaqin (Institut } \\
\text { Teknologi Sepuluh Nopember, Surabaya, Indonesia) }\end{array}$ & 257 \\
\hline $\begin{array}{l}\text { The Design a System of Retention and Control on Broiler Farms Based on } \\
\text { The Flow of Data } \\
\text { Ahmad Sanmorino, Isabella (Universitas Indo Global Mandiri, Palembang, } \\
\text { Indonesia) }\end{array}$ & 263 \\
\hline $\begin{array}{l}\text { Empirical Investigation on Factors Related to Individual of Impact } \\
\text { Performance Information System } \\
\text { Tri Lathif Mardi Suryanto, Nur Cahyo Wibowo (Universitas Pembangunan } \\
\text { Nasional “Veteran" Jawa Timur), Djoko Budiyanto Setyohadi(Universitas Atma } \\
\text { Jaya Yogyakarta, Indonesia) }\end{array}$ & 267 \\
\hline $\begin{array}{l}\text { Comparative Study of Web3D Standard Format to Determine the Base } \\
\text { Format for A Web3D Framework } \\
\text { Mursid W. Hananto, Ahmad Ashari, Khabib Mustofa (Universitas Gadjah Mada, } \\
\text { Yogyakarta, Indonesia) }\end{array}$ & 273 \\
\hline $\begin{array}{l}\text { Task-Technology Fit for Textile Cyberpreneur's Intention to Adopt Cloud- } \\
\text { Based M-Retail Application } \\
\text { Nik Zulkarnaen Khidzir, Wan Safra Diyana, Wan Abdul Ghani, Tan Tse Guan } \\
\text { (Faculty of Creative Technology and Heritage, Universiti Malaysia Kelantan, } \\
\text { Bachok, Malaysia), Mohammad Ismail (Faculty of Entrepreneurship and } \\
\text { Business, Universiti Malaysia Kelantan, Kota Bharu, Malaysia) }\end{array}$ & 279 \\
\hline $\begin{array}{l}\text { MAKASSAR SMART CITY OPERATION CENTER PRIORITY OPTIMIZATION } \\
\text { USING FUZZY MULTI-CRITERIA DECISION-MAKING }\end{array}$ & 285 \\
\hline
\end{tabular}




\begin{tabular}{|c|c|}
\hline $\begin{array}{l}\text { Fachrul Kurniawan, Supeno Mardi Susiki Nugroho, Mochamad Hariadi (Institut } \\
\text { Teknologi Sepuluh Nopember (ITS), Surabaya), Aji Prasetya Wibawa } \\
\text { (Universitas Negeri Malang), Munir (Universitas Pendidikan Indonesia, } \\
\text { Bandung, Indonesia) }\end{array}$ & \\
\hline $\begin{array}{l}\text { Ontology-Based Sentence Extraction for Answering Why-Question } \\
\text { A. A. I. N. Eka Karyawati (Department of Computer Science, Faculty of } \\
\text { Mathematics and Natural Sciences, Udayana University, Bali, Indonesia) }\end{array}$ & 290 \\
\hline $\begin{array}{l}\text { The Ontology-Based Methodology Phases To Develop Multi-Agent System } \\
\text { (OmMAS) }\end{array}$ & 296 \\
\hline $\begin{array}{l}\text { Arda Yunianta, Omar Obarukab, Norazah Yusof (King Abdulaziz University, } \\
\text { Rabigh, Saudi Arabia), Aina Musdholifah (Gadjah Mada University, Indonesia), } \\
\text { Nataniel Dengen, Haviluddin (Mulawarman University, Indonesia), Herlina } \\
\text { Jayadiyanti (UPN Veteran Yogyakarta, Indonesia), Mohd Shahizan Othman } \\
\text { (University Teknologi Malaysia, Malaysia). }\end{array}$ & \\
\hline $\begin{array}{l}\text { Scalability Measurement of Business Process Model Using Business } \\
\text { Processes Similarity and Complexity } \\
\text { Muhammad Ainul Yaqin, Riyanarto Sarno, Abd. Charis Fauzan (Informatics } \\
\text { Department, Institut Teknologi Sepuluh Nopember) }\end{array}$ & 302 \\
\hline $\begin{array}{l}\text { Smartphone for Next Generation Attendance System and Human } \\
\text { Resources Payroll System }\end{array}$ & 309 \\
\hline $\begin{array}{l}\text { Benfano Soewito, Fergyanto E. Gunawan (Binus Graduate Programs Bina } \\
\text { Nusantara University Jakarta, Indonesia), Manik Hapsara (University of New } \\
\text { South Wales Canberra, Australia) }\end{array}$ & \\
\hline $\begin{array}{l}\text { Enhancing Online Business Marketing to Expand Market Shares through } \\
\text { IT Governance }\end{array}$ & 315 \\
\hline $\begin{array}{l}\text { Sandy Kosasi, Vedyanto, I Dewa Ayu Eka Yuliani (Information System } \\
\text { Department STMIK Pontianak Pontianak, West Kalimantan, Indonesia) }\end{array}$ & \\
\hline A Generic Framework for Information Security Policy Development & 320 \\
\hline $\begin{array}{l}\text { Wan Basri Wan Ismail, Raja Ahmad Tariqi Raja Ahmad, Setyawan Widyarto } \\
\text { (Faculty of Communication, Visual Art and Computing University of Selangor } \\
\text { Malaysia), Khatipah Abd Ghani (Faculty of Education and Social Science } \\
\text { University of Selangor Malaysia) }\end{array}$ & \\
\hline Modeling IT Value based on Meta-Analysis & 326 \\
\hline $\begin{array}{l}\text { Suhardi, Novianto Budi Kurniawan, Aan Subrata, Jaka Sembiring (School of } \\
\text { Electrical Engineering and Informatics Institut Teknologi Bandung Bandung, } \\
\text { Indonesia) }\end{array}$ & \\
\hline $\begin{array}{l}\text { A Combination of The Evolutionary Tree Miner and Simulated Annealing } \\
\text { Afina Lina Nurlaili, Riyanarto Sarno (Department of Informatics Institut }\end{array}$ & 332 \\
\hline
\end{tabular}




\begin{tabular}{|c|c|}
\hline Teknologi Sepuluh Nopember Surabaya, Indonesia) & \\
\hline $\begin{array}{l}\text { Scalable Attack Analysis of Business Process based on Decision Mining } \\
\text { Classification } \\
\text { Dewi Rahmawati, Riyanarto Sarno (Informatics Department, Institut Teknologi } \\
\text { Sepuluh Nopember, Surabaya, Indonesia) }\end{array}$ & 337 \\
\hline $\begin{array}{l}\text { Service Computing System Engineering Life Cycle } \\
\text { Suhardi, Novianto Budi Kurniawan, Jaka Sembiring (School of Electrical } \\
\text { Engineering and Informatics, Institut Teknologi Bandung,Bandung, Indonesia) }\end{array}$ & 343 \\
\hline $\begin{array}{l}\text { High Efficiency Single Phase Inverter Design } \\
\text { Didi Istardi (Politeknik Negeri Batam, Indonesia) }\end{array}$ & 349 \\
\hline $\begin{array}{l}\text { Analysis of Electric Circuit Model on Atmospheric Pressure Dielectric } \\
\text { Barrier Discharge (DBD) Plasma } \\
\text { Suyadi, Jatmiko E Suseno, Muhammad Nur (Diponegoro University) }\end{array}$ & 352 \\
\hline $\begin{array}{l}\text { COMPRESSED NATURAL GAS (CNG) TECHNOLOGY FOR FUEL POWER } \\
\text { PLANTS } \\
\text { Isworo Pujotomo, Retno Aita Diantari (College of Engineering - PLN } \\
\text { Foundation for Education \& Welfare PT. PLN (Persero) Jakarta, Indonesia) }\end{array}$ & 352 \\
\hline $\begin{array}{l}\text { PID Designs Using DE and PSO Algorithms For Damping Oscillations in a } \\
\text { DC Motor Speed } \\
\text { Lailis Syafaah, Widianto, Ilham Pakaya, Diding Suhardi, M. Irfan (Department of } \\
\text { Electrical Engineering,The University of Muhammadiyah Malang, Indonesia) }\end{array}$ & 354 \\
\hline $\begin{array}{l}\text { Measurement of Partial Discharge Induced Electromagnetic Wave using } \\
\text { Loop Antenna } \\
\text { Umar Khayam, Fendi Imam Fatoni (School of Electrical Engineering and } \\
\text { Informatics, Bandung Institute of Technology, Bandung, Indonesia) }\end{array}$ & 359 \\
\hline $\begin{array}{l}\text { The Effect of Coating on Leakage Current Characteristic of Coast Field } \\
\text { Aged Ceramic Insulator } \\
\text { Dini Fauziah*, Heldi Alfiadi, Rachmawati, Suwarno (School of Electrical } \\
\text { Engineering and Informatics, Institut Teknologi Bandung, Bandung, Indonesia) }\end{array}$ & 363 \\
\hline $\begin{array}{l}\text { Renewable Energy Inclusion on Economic Power Optimization using } \\
\text { Thunderstorm Algorithm }\end{array}$ & 369 \\
\hline $\begin{array}{l}\text { A.N. Afandi (Universitas Negeri Malang, Jawa Timur, Indonesia), Goro Fujita, } \\
\text { Nguyen Phuc Khai (Shibaura Institute of Technology, Tokyo, Japan), Yunis } \\
\text { Sulistyorini (IKIP Budi Utomo, Malang, Indonesia), Nedim Tutkun (Duzce } \\
\text { University,Duzce, Tukey) }\end{array}$ & \\
\hline Optimum Phase Number for Multiphase PWM Inverters & 375 \\
\hline $\begin{array}{l}\text { Anwar Muqorobin, Pekik Argo Dahono and Agus Purwadi (School of Electrical } \\
\text { Engineering and Informatics, Institute of Technology Bandung, Bandung, } \\
\text { Indonesia) }\end{array}$ & \\
\hline
\end{tabular}




\begin{tabular}{|c|c|}
\hline $\begin{array}{l}\text { Small-Disturbance Angle Stability Enhancement using Intelligent Redox } \\
\text { Flow Batteries }\end{array}$ & \multirow[t]{3}{*}{381} \\
\hline $\begin{array}{l}\text { Mohammad Taufik (Padjadjaran University, Sumedang, Indonesia), Dwi } \\
\text { Lastomo (University of PGRI Adi Buana, Surabaya, Indonesia), Herlambang } \\
\text { Setiadi (School of Information Technology \& Electrical Engineering, The } \\
\text { University of Queensland }\end{array}$ & \\
\hline Brisbane, Australia) & \\
\hline $\begin{array}{l}\text { Evaluation Study of Waste Materials for Renewable Energy through 3R } \\
\text { Model in Bogor City }\end{array}$ & \multirow[t]{2}{*}{387} \\
\hline $\begin{array}{l}\text { Didik Notosudjono, Dede Suhendi, Engkos, Bagus Dwi Ramadhon (Electrical } \\
\text { Engineering Department, Universitas Pakuan, Bogor, Indonesia) }\end{array}$ & \\
\hline $\begin{array}{l}\text { Measurement of Partial Discharge inside Metal Enclosed Power Apparatus } \\
\text { using Internal Sensor }\end{array}$ & \multirow[t]{2}{*}{391} \\
\hline $\begin{array}{l}\text { Umar Khayam, Yushan (School of Electrical Engineering and Informatics } \\
\text { Bandung Institute of Technology Bandung, Indonesia) }\end{array}$ & \\
\hline $\begin{array}{l}\text { Design Unmanned Aerial Vehicle Integrated Camera Near Infra-Red to } \\
\text { Observe the Plant Health }\end{array}$ & \multirow[t]{2}{*}{397} \\
\hline $\begin{array}{l}\text { Rizki Wahyu Pratama, Ferry Hadary, Redi Ratiandi Yacoub (Jurusan Teknik } \\
\text { Elektro Fakultas Teknik Universitas Tanjungpura) }\end{array}$ & \\
\hline $\begin{array}{l}\text { Single Frame Resection of Compact Digital Cameras for UAV Imagery } \\
\text { Martinus Edwin Tjahjadi (Department of Geodesy, National Institute of } \\
\text { Technology (ITN) Malang, Indonesia) }\end{array}$ & 401 \\
\hline $\begin{array}{l}\text { A MOVING OBJECTS DETECTION IN UNDERWATER VIDEO USING } \\
\text { SUBTRACTION OF THE BACKGROUND MODEL } \\
\text { M. R. Prabowo, N. Hudayani, S. Purwiyanti, S. R. Sulistiyanti, F. X. A. } \\
\text { Setyawan (Department of Electrical Engineering, Faculty of Engineering } \\
\text { University of Lampung, Bandar Lampung, Indonesia) }\end{array}$ & 406 \\
\hline $\begin{array}{l}\text { Fall Detection Based on Accelerometer and Gyroscope using Back } \\
\text { Propagation } \\
\text { Adlian Jefiza (Institut Teknologi Sepuluh Nopember, Surabaya Indonesia) }\end{array}$ & 410 \\
\hline Honey Yield Prediction Using Tsukamoto Fuzzy Inference System & \multirow[t]{2}{*}{416} \\
\hline $\begin{array}{l}\text { Tri Hastono, Albertus Joko Santoso, Pranowo(Universitas Atma Jaya } \\
\text { Yogyakarta, Indonesia) }\end{array}$ & \\
\hline $\begin{array}{l}\text { Determining The Nutrition of Patient Based on Food Packaging Product } \\
\text { Using Fuzzy C Means Algorithm }\end{array}$ & \multirow[t]{2}{*}{422} \\
\hline $\begin{array}{l}\text { Sri Winiarti, Sri Kusumadewi, Izzati Muhimmah, Herman Yuliansyah } \\
\text { (Universitas Ahmad Dahlan Yogyakarta, Indonesia) }\end{array}$ & \\
\hline
\end{tabular}


The Successful Elements Implementing the eLearning using Cloud Services Data Centre at Private Institution of Higher Learning in Malaysia

Azlinda Abdul Aziz, Setyawan Widyarto, Salyani Osman, Suziyanti Marjudi (Department of Computing, Faculty of Communication, Visual Art and Computing, UNISEL, Kuala Selangor, Malaysia)

Improving E-Book Learning Experience by Learning Recommendation Fergyanto E. Gunawan, Benfano Soewito (Binus Graduate Programs, Bina Nusantara University, Jakarta, Indonesia), and Sevenpri Candra (School of Business Management, Bina Nusantara University, Jakarta, Indonesia).

\section{A Comparison of Cloud Execution Mechanisms: Fog, Edge and Clone Cloud Computing}

Tina Francis (Computer Department, BITS Pilani, Dubai Campus, DIAC Dubai, UAE), Dr. Muthiya Madhiajagan (SCOPE (School of Computer Science and Engineering) VIT University, Vellore Tamil Nadu, India)

\section{Recommendation System on Knowledge Management System via OAl-} PMH

Nyoman Karna, Iping Supriana, Nur Maulidevi (Sekolah Teknik Elektro dan Informatika Institut Teknologi Bandung, Indonesia)

Development and Evaluation of Android Based Notification System to Determine Patient's Medicine for Pharmaceutical Clinic Imam Riadi, Sri Winiarti, Herman Yuliansyah (Department of Informatics Universitas Ahmad Dahlan Yogyakarta)

Implementation of Decision Expert (DEX) in The "SALADGARDEN" Application

Anita Hidayati, Fityan Aula Juyuspan, Cindy Novianty, Muhammad Bima D S (Computer and Informatics Enginering Jakarta State Polytechnic Depok, Indonesia)

Optimizing Effort and Time Parameters of COCOMO II Estimation using Fuzzy Multi-Objective PSO

Kholed Langsari, Riyanarto Sarno (Department of Informatics Engineering Institut Teknologi Sepuluh Nopember Surabaya, Indonesia)

Evaluation Of Knowledge Management System Using Technology Acceptance Model

Jarot S. Suroso, Astari Retnowardhani, Abraham Fernando (Bina Nusantara University Jakarta, Indonesia) advising students' study path 


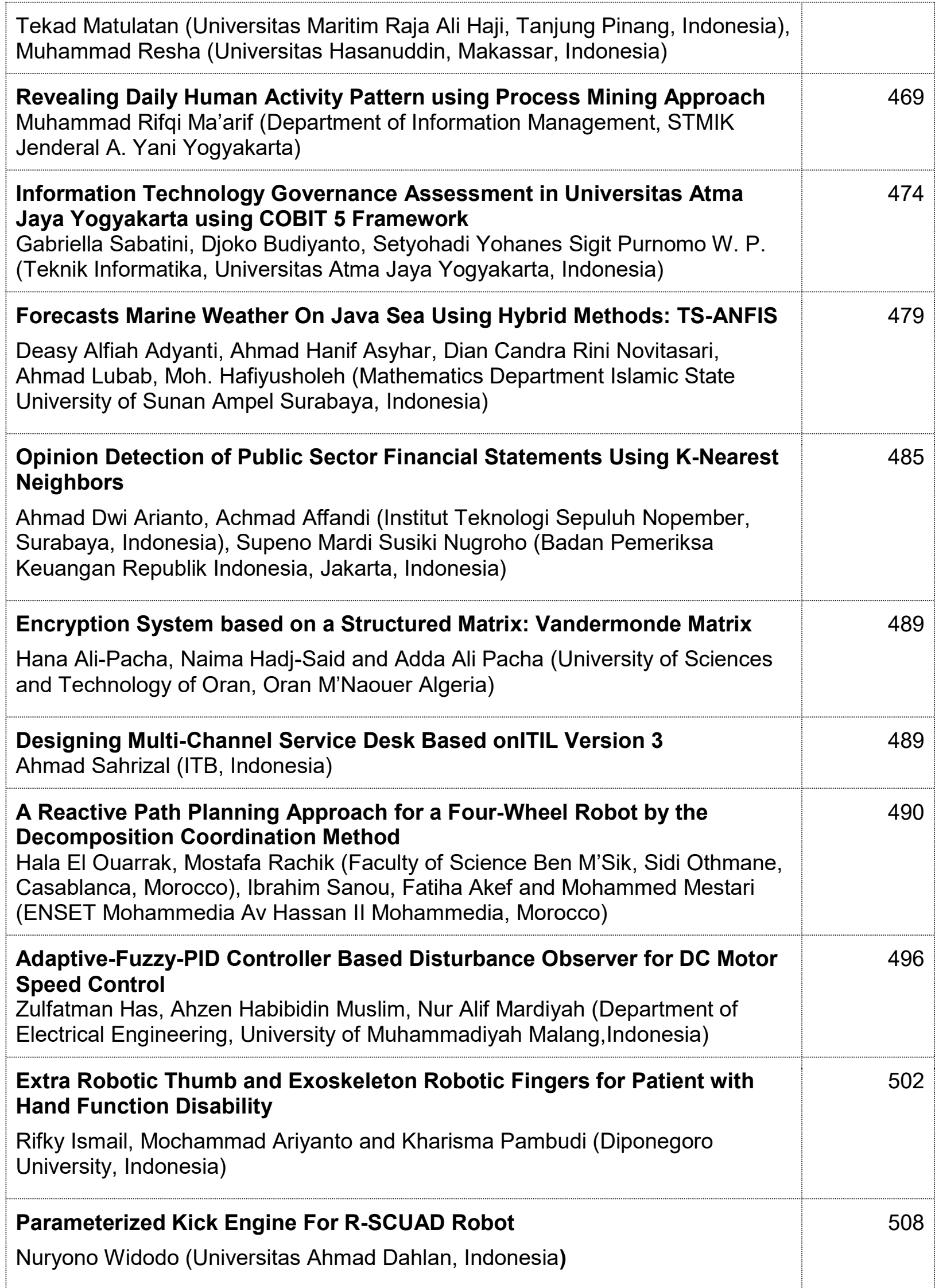




\begin{tabular}{|c|c|}
\hline $\begin{array}{l}\text { Neural Network Controller Design for a Mobile Robot Navigation; a Case } \\
\text { Study } \\
\text { Tresna Dewi, Pola Risma, Yurni Oktarina, and M. Taufik Roseno (Politeknik } \\
\text { Negeri Sriwijaya Palembang, Indonesia) }\end{array}$ & 512 \\
\hline $\begin{array}{l}\text { Redirection Concept of Autonomous Mobile Robot HY-SRF05 Sensor to } \\
\text { Reduce The Number of Sensors } \\
\text { Nuryanto, Andi Widiyanto, Auliya Burhanuddin (Engineering Faculty, } \\
\text { Universitas Muhammadiyah Magelang, Indonesia) }\end{array}$ & 517 \\
\hline $\begin{array}{l}\text { Autonomous Navigation for an Unmanned Aerial Vehicle by the } \\
\text { Decomposition Coordination Method } \\
\text { Chaimaa Jihane } \square \text {, Hala El Ouarrak } \dagger \text {, and Mohamed Mestari } \square \text {, Mostafa } \\
\text { Rachik } \dagger \text { ( } \square \text { Ecole Normale Suprieure d'Enseignement Technique } \\
\text { Mohammedia, Av Hassan II Mohammedia, Morocco, †Faculty of Science Ben } \\
\text { M'Sik, Casablanca, Morocco) }\end{array}$ & 521 \\
\hline $\begin{array}{l}\text { Design of PID Disturbance Observer for Temperature Control on Room } \\
\text { Heating System } \\
\text { Yoga Alif Kurnia Utama (Electronic Engineering Department, University of } \\
\text { Widya Kartika, Surabaya, Indonesia) }\end{array}$ & 527 \\
\hline $\begin{array}{l}\text { Development of Low Cost Supernumerary Robotic Fingers as an Assistive } \\
\text { Device }\end{array}$ & 533 \\
\hline $\begin{array}{l}\text { Mochammad Ariyanto*, Rifky Ismail, Joga Dharma Setiawan, Zainal Arifin } \\
\text { (Department of Mechanical Engineering, Diponegoro University, Semarang, } \\
\text { Indonesia) }\end{array}$ & \\
\hline $\begin{array}{l}\text { Design of A Microchip Optical Switching Driven by Low Direct-Current } \\
\text { Voltage }\end{array}$ & 539 \\
\hline $\begin{array}{l}\text { Dedi Irawan (Islamic State University of Sultan Syarif Kasim Riau, Pekanbaru, } \\
\text { Indonesia) }\end{array}$ & \\
\hline $\begin{array}{l}\text { A Web-Based Wireless Sensor System to Measure Carbon Monoxide } \\
\text { Concentration }\end{array}$ & 544 \\
\hline $\begin{array}{l}\text { Suryono, Ragil Saputra, Bayu Surarso, Ali Bardadi (Diponegoro University, } \\
\text { Semarang, Indonesia) }\end{array}$ & \\
\hline $\begin{array}{l}\text { Detecting the Early Drop of Attention using EEG Signal } \\
\text { Fergyanto E. Gunawan, Krisantus Wanandi, Benfano Soewito (Binus Graduate } \\
\text { Programs, Bina Nusantara University, Jakarta, Indonesia), Sevenpri Candra } \\
\text { (School of Business Management, Bina Nusantara University) }\end{array}$ & 549 \\
\hline $\begin{array}{l}\text { The Design of a Smart Refrigerator Prototype } \\
\text { Z. Ali, S. E. Esmaeili (Department of Electrical and Computer Engineering, } \\
\text { American University of Kuwait, Salmiya, Kuwait) }\end{array}$ & 554 \\
\hline Odor Localization using Gas Sensor for Mobile Robot & 555 \\
\hline
\end{tabular}


Nyayu Latifah Husni, Ade Silvia Handayani (State Polytechnic of Sriwijaya, Palembang, Indonesia), Siti Nurmaini, Irsyadi Yani (University of Sriwijaya, Palembang, Indonesia)

A Project-Based Approach to FPGA-Aided Teaching of Digital Systems Fajar Suryawan (Universitas Muhammadiyah Surakarta, Indonesia)

Performance of Routing Protocol in MANET with Combined Scalable Video Coding

Parma Hadi Rantelinggi, Fridolin Febrianto Paiki (Universitas Papua Manokwari, Indonesia), Kalvein Rantelobo (Universitas Nusa Cendana Kupang, Indonesia)

Attack Scenarios and Security Analysis of MQTT Communication Protocol in loT System

Syaiful Andy, Budi Rahardjo, Bagus Hanindhito (Institut Teknologi Bandung, Bandung, Indonesia)

Encoding of passive anticollision radio-frequency identification surface acoustic waves tags

Alexander Sorokin, Alexander Shepeta, Maurits Wattimena (Major ProblemOriented Computer Complexes Department, State University of Aerospace Instrumentation (SUAI), St. Petersburg, Russia)

Compact Fractal Patch Microstrip Antenna Fed by Coplanar Waveguide for Long Term Evolution Communications Indra Surjati (Universitas Trisakti , Jakarta, Indonesia)

Graphical Approach for RF Amplifier Specification in Radio over Fiber System: Maximum Power Issues

Teguh Prakoso, Munawar Agus Riyadi (Universitas Diponegoro, Semarang, Indonesia), Razali Ngah (Universiti Teknologi Malaysia, Johor Bahru, Malaysia)

FEM Modeling of Squeeze Film Damping Effect in RF-MEMS Switches Syed Turab Haider (Department of Electrical Engineering, National University of Sciences and Technology, Islamabad, Pakistan)

The Onion Routing Performance using Shadow-plugin-TOR Hartanto Kusuma Wardana, Liauw Frediczen Handianto, Banu Wirawan Yohanes* (Faculty of Electronic and Computer Engineering, Universitas Kristen Satya Wacana, Salatiga, Indonesia)

Position Tracking for Static Target using Burst Signals with Time Difference of Arrival Method

Romi Wiryadinata, Alia Shaliha Amany, Imamul Muttakin (Department of Electrical Engineering, University of Sultan Ageng Tirtayasa, Cilegon, Indonesia)

Performance Analysis for MIMO LTE on the High Altitude Platform Station

Catur Budi Waluyo, Yenni Astuti (Department of Electrical Engineering, Sekolah Tinggi Teknologi Adisutjipto, Yogyakarta, Indonesia) 


\begin{tabular}{|c|c|}
\hline $\begin{array}{l}\text { Software Defined Radio Design for OFDM Based Spectrum Exchange } \\
\text { Information Using Arduino UNO and X-Bee }\end{array}$ & \multirow[t]{2}{*}{608} \\
\hline $\begin{array}{l}\text { Arief Marwanto (Univ. Islam Sultan Agung (UNISSULA) Semarang - } \\
\text { Indonesia), Sharifah Kamilah Syed Yusof, Muhammad Haikal Satria (Universiti } \\
\text { Teknologi Malaysia (UTM) Johor Bahru - Malaysia) }\end{array}$ & \\
\hline $\begin{array}{l}\text { Performance Rate for Implementation of Mobile Learning in Network } \\
\text { Edy Budiman, Usfandi Haryaka, Jefferson Roosevelt Watulingas (Universitas } \\
\text { mulawarman, Samarinda - Indonesia), Faza Alameka (Universitas Ahmad } \\
\text { Dahlan, Yogyakarta - Indonesia) }\end{array}$ & 613 \\
\hline $\begin{array}{l}\text { Performance Evaluation of IPv6 Jumbogram Packets Transmission using } \\
\text { Jumbo Frames }\end{array}$ & \multirow[t]{2}{*}{619} \\
\hline $\begin{array}{l}\text { Supriyanto, Rian Sofhan, Rian Fahrizal (Department of Electrical Engineering, } \\
\text { University of Sultan Ageng Tirtayasa, Indonesia), Azlan Osman (School of } \\
\text { Computer Sciences, Universiti Sains Malaysia, Penang, Malaysia) }\end{array}$ & \\
\hline $\begin{array}{l}\text { Performance Analysis of CSI:T Routing in a Delay Tolerant Networks } \\
\text { Hardika Kusuma Putri, Leanna Vidya Yovita, and Ridha Muldina Negara } \\
\text { (Telkom University, Bandung, Indonesia) }\end{array}$ & 624 \\
\hline $\begin{array}{l}\text { A Study of the Number of Wavelengths Impact in the Optical Burst } \\
\text { Switching Core Node }\end{array}$ & \multirow[t]{2}{*}{630} \\
\hline $\begin{array}{l}\text { Hani A. M. Harb (Faculty of CSIT, Baha University, AL-Baha, Saudi Arabia), } \\
\text { Waleed M. Gaballah (Al-Baha Private College of Science, AL-Baha, Saudi } \\
\text { Arabia), Ahmed S. Samra Ahmed Abo-Taleb (Mansoura University, Egypt), } \\
\text { Arief Marwanto (Sultan Agung Islamic Univ. Semarang, Indonesia) }\end{array}$ & \\
\hline $\begin{array}{l}\text { A Reconfigurable MIMO Antenna System for Wireless Communications } \\
\text { Evizal Abdul Kadir (Department of Information Technology, Faculty of } \\
\text { Engineering, Universitas Islam Riau, Pekanbaru, Riau, Indonesia) }\end{array}$ & 634 \\
\hline $\begin{array}{l}\text { Conceptual Framework for Public Policymaking based on System } \\
\text { Dynamics and Big Data } \\
\text { Feldiansyah Bin Bakri Nasution, Nor Erne Nazira Bazin (Universiti Teknologi } \\
\text { Malaysia (UTM) Johor Bahru, Malaysia), Hasanuddin (Faculty of Social and } \\
\text { Politic Sciences, Riau University, Indonesia) }\end{array}$ & 638 \\
\hline $\begin{array}{l}\text { Discovering Process Model from Event Logs by Considering Overlapping } \\
\text { Rules }\end{array}$ & \multirow[t]{2}{*}{645} \\
\hline $\begin{array}{l}\text { Yutika Amelia Effendi, Riyanarto Sarno (Department of Informatics Faculty of } \\
\text { Information Technology, Institut Teknologi Sepuluh Nopember, Surabaya, } \\
\text { Indonesia) }\end{array}$ & \\
\hline
\end{tabular}


CHMM for Discovering Intentional Process Model From Event Logs By Considering Sequence of Activities

Kelly R. Sungkono, Riyanarto Sarno ( Department of Informatics Engineering, Institut Teknologi Sepuluh Nopember, Surabaya, Indonesia)

Sosio-Technical Factors of E-Government Implementation

Darmawan Napitupulu (LIPI, Indonesia); Dana Sensuse (Laboratory of E-

Government, Indonesia); Yudho Sucahyo (University of Indonesia, Indonesia)

\section{Methodology for Constructing Form Ontology}

U. Ungkawa, D. H. Widyantoro \& B. Hendradjaya (School of Electrical Engineering and Informatics, Institut Teknologi Bandung, Bandung, Indonesia)

Integration Protocol Student Academic Information to Campus RFID Gate Pass System

Hendra Gunawan and Evizal Abdul Kadir (Department of Information

Technology, Faculty of Engineering, Universitas Islam Riau, Pekanbaru, Riau, Indonesia)

E-Learning Model for Equivalency Education Program in Indonesia Mesra Betty Yel (Faculty of Computer Science, STIKOM CKI, Jakarta, Indonesia), Sfenrianto (Master in Information Systems Management, Bina Nusantara University, Jakarta, Indonesia)

Developing E-Government Maturity Framework Based on COBIT 5 and Implementing in City Level:Case Study Depok City and South Tangerang City

Fikri Akbarsyah Anza (Public Administration Department, Universitas Indonesia), Dana Indra Sensuse, Arief Ramadhan (Computer Science Department, Universitas Indonesia)

Analysis of Driving Skills based on Deep Learning using Stacked Autoencoders

Takuya Kagawa, Naiwala P. Chandrasiri (Faculty of Information Kogakuin University, Tokyo, Japan)

Minimizing the Estimated Solution Cost with A* Search to Support Minimal Mapping Repair

Inne Gartina Husein*, Benhard Sitohang, Saiful Akbar (Institut Teknologi Bandung)

The rule Extraction of Numerical Association Rule Mining Using Hybrid Evolutionary Algorithm Imam Tahyudin (Kanazawa University, Japan)

Discovering Drugs Combination Pattern Using FP- Growth Algorithm

Rini Anggrainingsih, Nach Rowi Khoirudin, Haryono Setiadi (Informatics Dept Mathematics and Natural Science, UNS Surakarta, Indonesia ) 


\begin{tabular}{|c|c|}
\hline $\begin{array}{l}\text { Classifiers Evaluation: Comparison of Performance Classifiers Based on } \\
\text { Tuples Amount } \\
\text { Mochammad Yusa, Ema Utami (Department of Computer Science, Magister } \\
\text { Teknik Informatika Universitas AMIKOM Yogyakarta, Indonesia) }\end{array}$ & 706 \\
\hline $\begin{array}{l}\text { Prediction of Rupiah Against US Dollar by Using ARIMA } \\
\text { Adiba Qonita, Annas Gading Pertiwi, Triyanna Widiyaningtyas (Electrical } \\
\text { Engineering Department, Universitas Negeri Malang, Malang, Indonesia) }\end{array}$ & 713 \\
\hline $\begin{array}{l}\text { Forming Heterogeneous Group in Cooperative Learning Process using } \\
\text { Partitioning Around Medoids (PAM) and Equitable Distribution } \\
\text { Imam Much Ibnu Subroto, Badieah Assegaf and Wardianto Eko Saputra } \\
\text { (Universitas Islam Sultan Agung, Indonesia) }\end{array}$ & 718 \\
\hline
\end{tabular}




\section{Papers by title}

\section{A C D E F G H I M N O P R S T U V W}

\section{2 ACDEFGHIMNOPRSTUVW}

2D-Sigmoid Enhancement Prior to Segment MRI Glioma Tumour

\section{A 2 A C DEF G H I M NOPRST U V W}

A Combination of The Evolutionary Tree Miner and Simulated Annealing

A Comparison on Cloud Execution Mechanisms: Fog, Edge and Clone cloud Computing

A Generic Framework for Information Security Policy Development

A Hierarchical Description-based Video Monitoring System for Elderly

A Moving Objects Detection in Underwater Video Using Substraction of the Background Model

A Project-Based Approach to FPGA-Aided Teaching of Digital Systems

A Reactive path planning approach for a Four-Wheel Robot by the Decomposition Coordination Method

A Reconfigurable MIMO Antenna System for Wireless Communications

A Study of the Number of Wavelengths Impact in the Optical Burst Switching Core Node

A Web-Based Wireless Sensor System to Measure Carbon Monoxide Concentration

Adaptive-Fuzzy-PID Controller Based Disturbance Observer for DC Motor Speed Control

Alerting System for Sport Activity Based on ECG Signals using Proportional Integral Derivative

An Analysis of Concentration Region on Powerpoint Slides using Eye Tracking

Analysis of Driving Skills based on Deep Learning using Stacked Autoencoders

Analysis of Electric Circuit Model on Atmospheric Pressure Dielectric Barrier Discharge (DBD) Plasma

Analysis of Holt-Winters Exponential Smoothing and Moving Average Model For Forecasting Ritase

Analysis of Statement Branch and Loop Coverage in Software Testing With Genetic Algorithm

Analysis of the Indonesian Vowel /e/ For Lip Synchronization Animation

Anti-Cheating Presence System Based on 3WPCA-Dual Vision Face Recognition

Attack Scenarios and Security Analysis of MQTT Communication Protocol in IoT System

Attack Scenarios and Security Analysis of MQTT Communication Protocol in IoT System

Automated Post-Trabeculectomy Bleb Assesment by Using Image Processing

Autonomous navigation for an Unmanned Aerial Vehicle by the Decomposition Coordination Method

\section{2 ACDEF G H I N N P R T U V W}

Certain Factor Analysis for Extra Pulmonary Tuberculosis Diagnosis

CHMM for Discovering Intentional Process Model From Event Logs By Considering Sequence of Activities

Classifiers Evaluation: Comparison of Performance Classifiers Based on Tuples Amount

Combining Deep Belief Networks and Bidirectional Long Short Term Memory (Case Study: Sleep Stages Classification) 
Compact Fractal Patch Microstrip Antenna Fed by Coplanar Waveguide for Long Term Evolution Communications Comparative Study of Web3D Standard Format to Determine the Base Format for A Web3D Framework Compressed Natural Gas (CNG) Technology For Fuel Power Plants

Computer Vision Based Object Tracking as a Teaching Aid for High School Physics Experiments Conceptual Framework for Public Policymaking based on System Dynamics and Big Data

\section{2 A C D E F G H I N O PRST U V W}

Deep learning on curriculum study pattern by selective cross join in advising students' study path

Design of A Microchip Optical Switching Driven by Low Direct-Current Voltage

Design of Automatic Switching Bio-Impedance Analysis (BIA) for Body Fat Measurement

Design of PID Disturbance Observer for Temperature Control on Room Heating System

Design Unmanned Aerial Vehicle Integrated Camera Near Infra-Red to Observe the Plant Health

Designing Multi-Channel Service Desk Based onITIL Version 3

Detecting the Early Drop of Attention using EEG Signal

Determining The Nutrition of Patient Based on Food Packaging Product Using Fuzzy C Means Algorithm

Developing E-Government Maturity Framework Based On COBIT 5

Development and Evaluation of Android Based Notification System to Determine Patient's Medicine

Development of Low Cost Supernumerary Robotic Fingers as an Assistive Device

Discovering Drugs Combination Pattern Using FP-Growth Algorithm

Discovering Process Model from Event Logs by Considering Overlapping Rules

\section{E 2 A C D E F G H I M N O PRST U V W}

E-learning Model for Equivalency Education Program in Indonesia

Edge Detection on Objects of Medical Image with Enhancement multiple Morphological Gradient Method EEG Based Emotion Monitoring Using Wavelet and Learning Vector Quantization

Effect of the Presence of Metal Box on Partial Discharge Detected by Internal Loop Sensor

Empirical Investigation on Factors Related to Individual of Impact Performance Information System

Empirical Investigation on Factors Related to Individual of Impact Performance Information System

Encoding Of Passive Surface Acoustic Wave Based Anti-Collision Radio Frequency Identification Tags

Encryption System based on a Structured Matrix: Vandermonde Matrix

Enhancing Online Business Marketing to Expand Market Shares through IT Governance

Ethnobotany Database: Information Management and Exploration of Medicinal Plants Borneo

Evaluation Of Knowledge Management System Using Technology Acceptance Model

Evaluation Study of Waste Materials for Renewable Energy through 3R Model in Bogor City

Extra Robotic Thumb and Exoskeleton Robotic Fingers for Patient with Hand Function Disability 


\section{F $\quad$ TOP D E F G H I M N O P R S T U V W}

Fall Detection Based on Accelerometer and Gyroscope using Back Propagation

Feature Extraction and Classification of Thorax X-Ray Image in The Assessment of Osteoporosis

FEM Modeling of Squeeze Film Damping Effect in RF-MEMS Switches

Forecasts Marine Weather On Java Sea Using Hybrid Methods: TS-ANFIS

Forming Heterogeneous Group in Cooperative Learning Process using PAM and Equitable Distribution

\section{G 2 A C D E F G H I M NOPRST U V W}

Graphical Approach for RF Amplifier Specification in Radio over Fiber System: Maximum Power Issues Graphical Approach for RF Amplifier Specification in Radio over Fiber System: Maximum Power Issues

\section{H 2 A C D E F G I M NOPRSTU V W}

High Efficiency Single Phase Inverter Design

High Performance Direct Torque Control of Induction Motor Drives: Problems and Improvements Honey Yield Prediction Using Tsukamoto Fuzzy Inference System

\section{I $\quad$ T A C D E F G I M NOPRST U V W}

Imperceptible Image Watermarking based on Chinese Remainder Theorem over the Edges

Implementation of Decision Expert (DEX) in The "SALADGARDEN" Application

Implementation of K-Means Clustering Method to Distribution of High School Teachers

Implementation of Unbiased Stereology Method for Organ Volume Estimation using Image Processing

Improvement of eGov \& mGov in Multilingual Countries with Digital Etymology using Sanskrit Grammar

Improving E-Book Learning Experience by Learning Recommendation

Incremental High Throughput Network Traffic Classifier

Integration Protocol Student Academic Information to Campus RFID Gate Pass System

IoT Smart Device for e-Learning Content Sharing on Hybrid Cloud Environment

\section{2 ACDEFG I M NOPRST U V W}

Makassar Smart City Operation Centre Priority Optimization Using Fuzzy MCDM

Measurement Of Maximum Value Of Dental Radiograph To Predict The Bone Mineral Density

Measurement of Partial Discharge Induced Electromagnetic Wave using Loop Antenna

Measurement of Partial Discharge inside Metal Enclosed Power Apparatus using Internal Sensor

Methodology for Constructing Form Ontology

Minimizing the Estimated Solution Cost with $A^{*}$ Search to Support Minimal Mapping Repair

Mobile Application Design For Recommendation System On Disease Pattern Counseling

Mobile Content Based Image Retrieval Architectures 
Modeling IT Value based on Meta-Analysis

Myoelectric control systems for hand rehabilitation device: a review

\section{N 2 A C D E F G I M NOPRSTUVW}

Neural Network Controller Design for a Mobile Robot Navigation; a Case Study

Neural Network on Mortality Prediction for the Patient Admitted with ADHD

Nitrogen (N) Fertilizer Measuring Instrument On Maize-Based Plant Microcontroller

Non-invasive Hemoglobin Measurement for Anemia Diagnosis

\section{ACDEF G H I M N P R S T U V W}

Odor Localization using Gas Sensor for Mobile Robot

Ontology-Based Sentence Extraction for Answering Why-Question

Opinion Detection of Public Sector Financial Statements Using K-Nearest Neighbors

Optimization Syntax Query of Search Algorithm on Employee Pages Module Knowledge Management System

Optimizing Effort and Time Parameters of COCOMO II Estimation using Fuzzy Multi-Objective PSO

Optimum Phase Number for Multiphase PWM Inverters

\section{P 2 ACDEFGHIMNOPRSTUVW}

Parameterized Kick Engine For R-SCUAD Robot

Performance Analysis for MIMO LTE on the High Altitude Platform Station

Performance Analysis of CSI:T Routing in a Delay Tolerant Networks

Performance Analysis of Network Emulator Based On The Use of Resources In Virtual Laboratory

Performance Evaluation of IPv6 Jumbogram Packets Transmission using Jumbo Frames

Performance Measurement Based on Coloured Petri Net Simulation of Scalable Business Processes

Performance Measurement Based on Coloured Petri Net Simulation of Scalable Business Processes

Performance of Routing Protocol in MANET with Combined Scalable Video Coding

Performance of Routing Protocol in MANET with Combined Scalable Video Coding

Performance Rate for Implementation of Mobile Learning in Network

PID Designs Using DE and PSO Algorithms for Damping Oscillations in A DC Motor Speed

Poincaré plot of fingertip photoplethysmogram pulse amplitude suitable to assess diabetes status

Position Tracking for Static Target using Burst Signals with Time Difference of Arrival Method

Precise Wide Baseline Stereo Image Matching for Compact Digital Cameras

Prediction of Rupiah Against US Dollar by Using ARIMA

Problem Identification Of IT Governance Using COBIT 5 Framework For Education Information System

\section{R $\quad$ TACDEFGHIMNOPRSTUVW}

Recommendation System on Knowledge Management System via OAI-PMH 
Reconfigurable Logic Embedded Architecture of Support Vector Machine Linear Kernel

Redirection Concept of Autonomous Mobile Robot HY-SRF05 Sensor to Reduce The Number of Sensors

Region of Interest Detection for Pregnancy Image Processing

Renewable Energy Inclusion on Economic Power Optimization using Thunderstorm Algorithm

Research of Maneuver Target Tracking Filtering Algorithm

Research of Simple Multi-Attribute Rating Technique for Decision Support

Revealing Daily Human Activity Pattern using Process Mining Approach

Robust and Imperceptible Image Watermarking by DC Coefficients Using Singular Value Decomposition

Robust and Imperceptible Image Watermarking by DC Coefficients Using Singular Value Decomposition

\section{S 2 ACDEFGHIMNOPRSTUVW}

Scalability Measurement of Business Process Model Using Business Processes Similarity and Complexity

Scalable Attack Analysis of Business Process based on Decision Mining Classification

SDR Design for OFDM Based Spectrum Exchange Information Using Arduino UNO and X-Bee

Service Computing System Engineering Life Cycle

Shape Defect Detection for Product Quality Inspection and Monitoring System

Single Frame Resection of Compact Digital Cameras for UAV Imagery

Single Phase Inverter for Photovoltaic Source With Totem Pole Circuit

Sketch Plus Colorization Deep Convolutional Neural Networks for Photos Generation from Sketches

Small-Disturbance Angle Stability Enhancement using Intelligent Redox Flow Batteries

Smartphone for Next Generation Attendance System and Human Resources Payroll System

Sosio-Technical Factors of E-Government Implementation

Spoken Word Recognition Using Mel-Frequency Cepstrum Coefficients and Learning Vector Quantization

\section{T 2 ACDEFGHIMNOPRSTUVW}

Target Tracking in Mobile Robot under Uncertain Environment using Fuzzy Logic Controller

Task-Technology Fit for Textile Cyberpreneur's Intention to Adopt Cloud-Based M-Retail Application

Teaching \& Learning Support For COA Courses Design On CE \& CS For Undergraduate

Teaching \& Learning Support For COA Courses Design On CE \& CS For Undergraduate

Text Modeling In Adaptive Educational Chat Room Based On Madamira Tool

Texture Analysis and Fracture Identification of Lower Extremities Bones X-Ray Images

The design a system of retention and control on broiler farms based on the flow of data

The design a system of retention and control on broiler farms based on the flow of data

The Design of A Smart Refrigerator Prototype

The Effect of Coating on Leakage Current Characteristic of Coast Field Aged Ceramic Insulator

The Improvement of Phonocardiograph Signal (PCG) Representation Through the Electronic Stethoscope 
The Improvement of The Triple Play Services Function by The Gigabit-capable Passive Optical Networks The Onion Routing Performance using Shadow-plugin-TOR

The Ontology-Based Methodology Phases To Develop Multi-Agent System (OmMAS)

The rule Extraction of Numerical Association Rule Mining Using Hybrid Evolutionary Algorithm

The successful elements implementing the eLearning using Cloud Services Data Centre

Toward a New Approach in Fruit Recognition using Hybrid RGBD Features and Fruit Hierarchy Property

Towards Development of A Computerised System for Screening and Monitoring of Diabetic Retinopathy

\section{U 2 A C D E F G H I M NOPRST U V W}

Unified Concept-based Multimedia Information Retrieval Technique

\section{2 ACDEFG H I M NOPRST U V W}

Variance Analysis of Photoplethysmography for Blood Pressure Measurement

\section{W 2 A C D E F G H I M NOPRST U V W}

WatsaQ: Repository of Al Hadith in Bahasa (Case Study: Hadith Bukhari)

Wood Texture Detection with Conjugate Gradient Neural Network Algorithm 


\title{
Parameterized Kick Engine For R-SCUAD Robot
}

\author{
Nuryono Satya Widodo \\ Watra Arsadiando \\ Arif Rahman \\ Muhammad Iqbalul Faiq Hatta \\ Electrical Engineering Department \\ Faculty of Industrial Technology, Universitas Ahmad Dahlan \\ Information System Department* \\ Faculty of Natural Science, Universitas Ahmad Dahlan \\ nuryono.sw@ee.uad.ac.id
}

\begin{abstract}
Humanoid Robot Soccer is an implementation of technology in robotics area that has the ability to mimic human activity to play football. Kicking is one of many abilities that the robot must have to play football nicely. The ability to kick in a various kind of kick also needed to enhance it's performance in the match.This research was conducted on a 20 degree of freedom humanoid robot soccer named R-SCUAD. The robot was equipped with 20 Dynamixel servo motors as the actuator, an arbotix-pro as a sub-controller and an Odroid XU4 as the main controller. The method developed in this research was based on Darwin-op framework with an enhancement especially on the kick engine. Experiment results showed that there was a corellations between the distance that the ball travelled due to a kick with leg the swing speed of the kick, it can be inferred that a greater swing speed value will yields a greater distance of ball travel. With the leg swing speed of $50 \mathrm{rpm}$ generate average distance $36.58 \mathrm{~cm}$ while with a speed of $300 \mathrm{rpm}$ generate an average distance of $329.62 \mathrm{~cm}$. Result also showed that the balancing system developed based on kick angle computation was able to maintain the robot stability up to $25^{\circ}$ of kick angle.
\end{abstract}

Keywords; Forward kinematics; Humanoid robot soccer; Parametric kick;

\section{INTRODUCTION}

Indonesian Robocup Socccer Humanoid League was the new title for Kontes Robot Cerdas Indonesia (KRCI) Robo Soccer Humanoid League (RSHL) that became an event to select Indonesia's reperesentative for the Robocup Intenational yearly event. Humanoid Robot Soccer was a form of technological implementation in the robotic field that has the ability to mimic human's activities in playing football/soccer. [1].

The robot also must posses an ability to "hear" referee's instructions, in this case using a wifi network communication system. The robot must have the ability to percept the field and all form of object that may present on the field. The Robot must walk on biped while maintaining its balance so it can locomote reliably. Many researchers try to cope this problem in their research, such as [2-6]. Those research aimed to have a better walking capabilities for the robot. This research mainly based on the research of Darwin-op framework [2], that propose an approach to have a robot that has the ability to walk, kick and do some kind of movement based on input from the USB camera attached to the robot.

To achieve those kind of abilities, the robot equipped with a configuration of component and hardware that enable the robot to move freely and achieve high level of dynamics. Inside the robot's torso an embedded pc was placed as the main controller, the sub controller was stacked on the mani controller. As the actuation the robot uses 20 pieces Dynamixel servo, webcam was used as the vision sensory input to get information from its surrounding. A 3 axis Gyroscope and 3 axis accelerometer also attached to the robot as its internal state sensory input. This research was focused on how to develop a parametric kick engine that enables the robot to has an infinite variations of kick motion. The Darwinop framework already has an ability to do kicking motions, but it uses a prerecorded handcrafted motion. In other word we would have to create every single type of kick or motion manually by hand, if we want to have a variety of kick. It was a very time consuming task to craft this kind of motions because we need to manually adjust the positions of every joint, record those posisition as pose, proceeded with altering some joint to have a movement, record as another pose, one after another and save those pose as a motion that could be called to be playback on the future.

This research was motivated by the need to develop a kick engine that not involving handcrafting a kicking motions as the original Darwin-op framework did. Ferreira Rui, [7] has done a research titled Development of an Omnidirectional Kick for a NAO Humanoid Robot in which the developed kick engine consist of 5 phase.

- Lean_Phase - This is when the robot shifts is center of mass onto support leg.

- Raise_Phase - Phase where the robot raises the kick foot off the ground.

- Kick_Phase - When the robot kicks the ball. This is the main phase.

- Return Phase - Phase when the robot reurn the leg without putting the foot on the ground

- UnRaise_Phase - This is when the robot shifts is center putting the kick foot on the ground. 


\section{RESEARCH METHODOLOGY}

\section{A. Hardware Configuration}

Generally speaking, the robot consist of main control, sub control and actuator, the robot's block diagram presented on figure number 1 below :

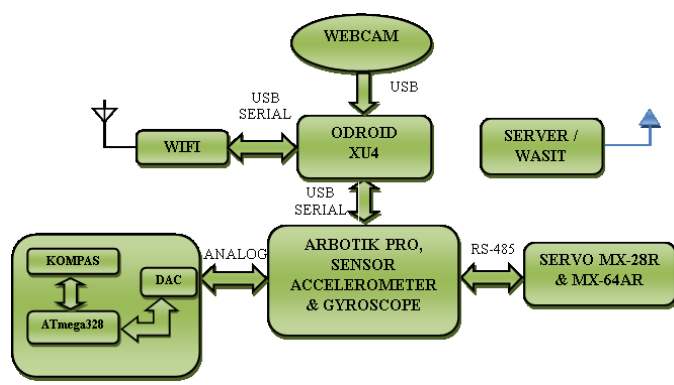

Figure 1 Robot's Block Diagram

The main controner was tne varoid $x \cup 4$. It s an AKIVI based single board computer with a Samsung Exynos5422 CortexA15 2Ghz and Cortex-A7 Octa core CPUs as the main processor. We use Linux Ubuntu 14.04 for this research and $\mathrm{C}++$ for the main programming language. OpenCV also installed on the main controller to utilize the library for the robot's vision system. Linux and android operating system for this board was made available by hardkernel as the creator of the board. As for the subcontroller we use a STM32 based controller, equipped with 3 axis gyroscope and 3 axis accelerometer and also the necessary circuitry needed to communicate to the Dynamixel MX series digital servomotor as the actuator. There R-SCUAD Robot used in this research, as shown in figure 2, use two kind of Dynamixel MX-series. The MX-64 and MX-28 series.

DYNAMIXEL (DXL) is a line-up of networked actuators for robots developed by a Korean manufacturer ROBOTIS. ROBOTIS is also the developer and manufacturer for DARwIn-OP[8]. DXL is being used by numerous companies, universities, and hobbyist due to its versatile expansion capability, powerful feedback functions, position, speed, internal temperature, input voltage, etc. It has a simple daisy-

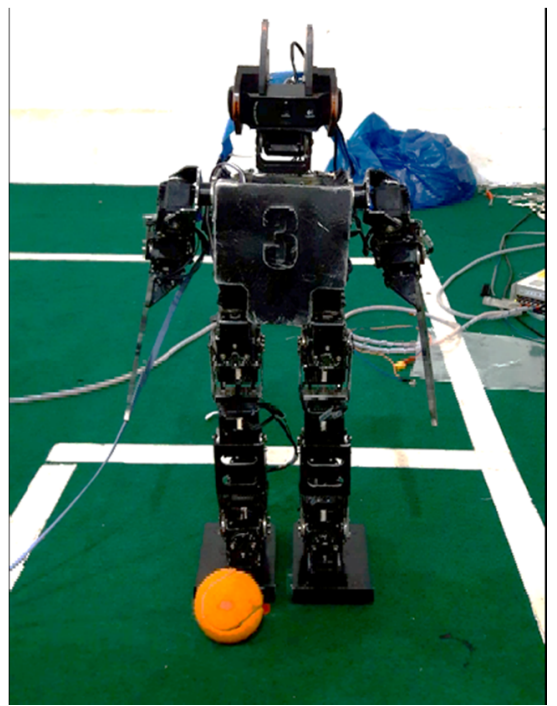

Figure 2 R-SCUAD Robot chain topology for simplified wiring connections.

DXL can be used for multi-joint robot systems such as robotic arms, robotic hand, bi-pedal robot, hexapod robot, snake robot scorpions, pan tilts, kinematic art, animatronics and automation, etc. Dynamixel is an actuator controlled by digital packet communication (protocol communication). User could send an instruction packet, in serial form, and the dynamixel will respond to the instruction accordingly.

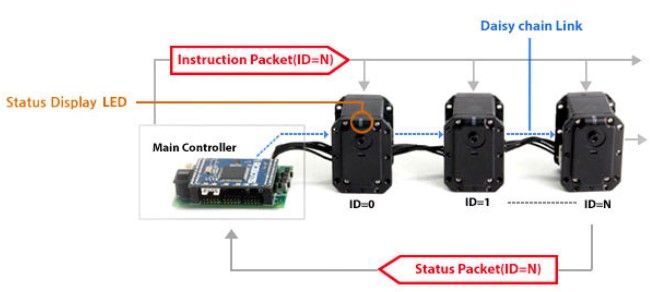

Figure 3 Dynamixel Communication Protocol

\section{B. Software Configuration}

The kick engine was developed based on forward kinematics, i.e. the system supplied with the joint position information and the final position of the link, the leg in this case was obtained. Simple balancing system based on the relation between kick angle and hand angle was added to kick engine. The balancing system will compute the necessary arm movement to encounter to force generated from opening the leg. The flowchart for the developed kick engine for the RSCUAD can be depicted on the figure 4., below.

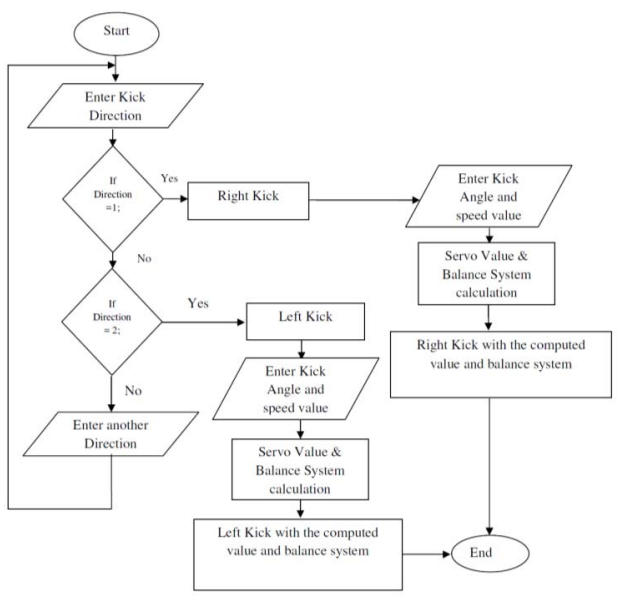

Figure 4 Flowchart of the developed kick engine 

kick.

A kick consist of 5 phase that must be completed to have a

- Lean

- Raise

- Kick

- Return

- UnRaise

Those stages can be depicted below

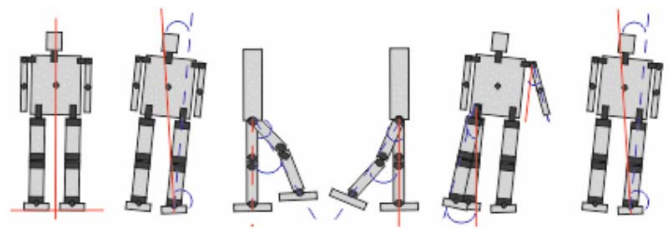

Figure 5 Kick Phase

\section{RESULT AND ANALYSIS}

There were two kind of testing in this research, first a test to verify distance traveled from a various kick swing speed, and the second was test to verify the robot stability for a numerous kind of kicking motions.

\section{A. Ball Travel}

As mentioned beforehand a kick consist of 5 phase, the implementation of those 5 phase can be seen on figure no 6 below
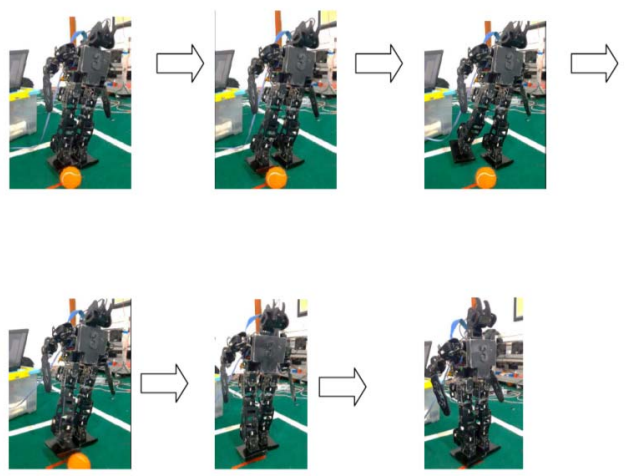

Figure 6 Kick Implementation

To verify that the kick engine developed has a controlling ability for ball travel distance, we conducted a series of test with the variation of kick swing speed. The result showed the relations between kick swing speed with the ball travel distance can be seen in figure 7 below

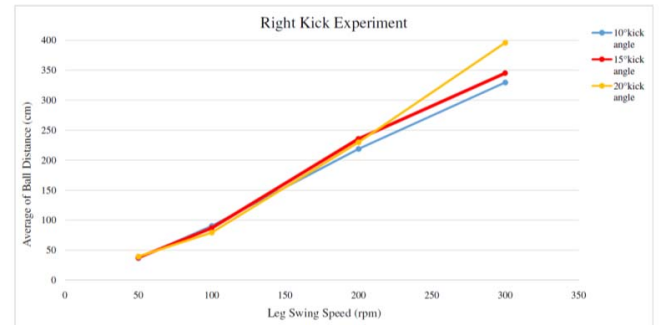

Figure 7 Ball Travel and Kick Speed
It can be inferred from the graph that the robot could control the ball travel distance proportionally with the kick swing speed for any given kick angle. Robot was able to kick the ball up to $3 \mathrm{~m}$ distance using $300 \mathrm{rpm}$ kick speed.

\section{B. Balancing Ability}

The balancing test was conducted using variation of kick angle and swing speed parameter. The parameter will be used as reference on how far the balancing system able to maintain the robot in standing position, i.e. not fall. There are two kind of test here, right kick and left kick. As mentioned earlier the balancing system was implemented simply using the kick angle, how far is the kicking leg opened from its normal position. This value will be used to determine how far the arm will move or opened to encounter the leg movement. This process is done synchronously while opening the leg.

Data collected for $10^{\circ}, 15^{\circ}, 20^{\circ}, 25^{\circ}$ and $30^{\circ}$ kick angle with $50,100,200$, and $300 \mathrm{rpm}$ kick speed, the result shown in figure no 8 . below.

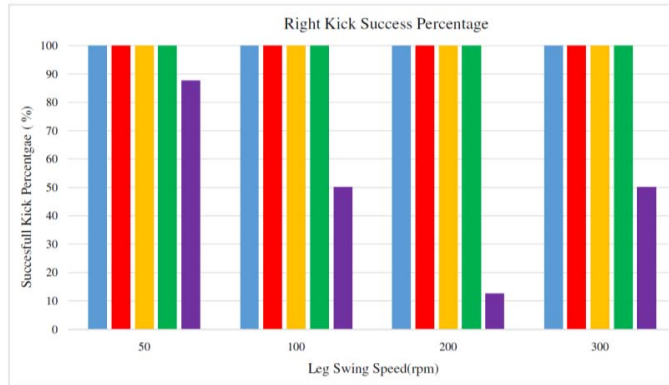

Figure 8 Succesful Kick Percentage (Right kick)

It can be seen from the graph that regarding right kick the robot has a very high percentage of successful kick motion for all kick swing speed, however there is a decrease in this percentage when we give a wider kick angle, in this case $30^{\circ}$.

Same procedure was taken for the left kick motion, the arm opening was done synchronously with the leg opening to maintain the robot's balance.

Data collected for $10^{\circ}, 15^{\circ}, 20^{\circ}, 25^{\circ}$ and $30^{\circ}$ kick angle with 50, 100, 200, and $300 \mathrm{rpm}$ kick speed, the result shown in figure no. 8 below.

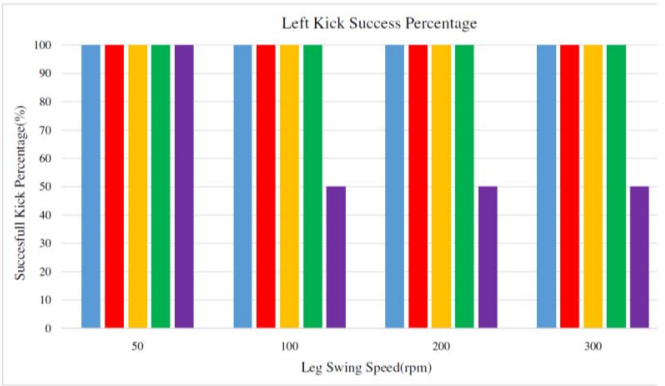

Figure 8 Succesful Kick Percentage (Left kick) 
It can be seen from the graph that regarding left kick the robot has a very high percentage of successful kick motion for all kick swing speed, however there is a decrease in this percentage when we give a wider kick angle, in this case $30^{\circ}$.

\section{CONCLUSION}

Based on experimental result, conclusions below could be inferred:

Kick variations were able to be implemented in the form of kick angle and kick swing speed parameter, these parameter were proportionally related to ball travel distance due to the kick. It were verified using $10^{\circ}, 15^{\circ}$ and $20^{\circ}$ kick angle and 50, 100, and $200 \mathrm{rpm}$ kick swing speed. The faster the kick swing the further the ball traveled. The developed balancing system was based on arm angle computation algorithm. The system was able to maintains the robot balance when performing a kicking motions. The developed balancing system still unable to guarantee a $100 \%$ secessful kick for $30^{\circ}$ of kick angle. Gyroscope and Accelerometer stabilized kick engine should be investigated in the future to enhace the robot balancing ability when performing a kicking motions.

\section{Acknowledgment}

The research was funded using Universitas Ahmad Dahlan Robotic team grant.

\section{References}

[1] Rule KRSBI (Kontes Robot Sepak Bola Indonesia) 2015 cited from https://www.kontesrobotindonesia.org/kri-2017/ (acessed January 03 2017)

[2] Ha, I., Tamura, Y., Asama, H., Han, J., Hong, D.: Development of open humanoid platform DARwIn-OP. In: SICE Annual Conf. (2011)

[3] Ha, I., Tamura, Y., Asama, H.,: Gait Pattern Generation and Stabilization for Humanoid Robot Based on Coupled Oscillators, 2011 IEEE/RSJ International Conference onIntelligent Robots and Systems

[4] Missura, M. and Behnke S. ,: Self-stable Omnidirectional Walking with Compliant Joints. In Proceedings of 8th Workshop on Humanoid Soccer Robots, IEEE-RAS International Conference on Humanoid Robots (Humanoids), Atlanta, October 2013.

[5] Karimi, M., Sadeghnejad S., Baltes J.: Online Omnidirectional Gait Modifications for a Full Body Push Recovery Control of a Biped Robot Proceeding of the 8thInternational Conference on Advanced Humanoid Robotics Research (ICAHRR)14th to 18th December 2016, Beijing, China

[6] Saputra, A.A., Botzheim J.: Biologically Inspired Control System for 3$\mathrm{D}$ Locomotion of a Humanoid Biped Robot Jan 2015 · IEEE Transactions on Systems, Man, and Cybernetics: Systems

[7] Ferreira Rui, dkk (2012), Development of an Omnidirectional Kick for a NAO Humanoid Robot

[8] http://support.robotis.com/en/product/actuator/dynamixel/dxl_mx_main. htm (accessed January 20 2017) 


\section{AUTHOR INDEX}

\begin{tabular}{|c|c|}
\hline Abd Ghani, Khatipah & D1RD.12 \\
\hline Abd. Rahim, Mohd. Rozaini & D1RA.4 \\
\hline Abdul Aziz, Azlinda & D2RA.2 \\
\hline Abdul Hamid, Abdul Hadi Fikri & D1RA.4 \\
\hline \multirow[t]{2}{*}{ Abdul Kadir, Evizal } & D2RC. 15 \\
\hline & D2RD.6 \\
\hline $\begin{array}{l}\text { Abdul Rahman, Nor Nabilah } \\
\text { Syazana }\end{array}$ & D1RC.4 \\
\hline Abdul Wahab, Farhan & D1RC.4 \\
\hline Abdullah, Abdul Rahim & D1RC.4 \\
\hline Abdullah, Khairul Annuar & D1RC.14 \\
\hline Abu Yazid, Mohamad Haider & D1RB.11 \\
\hline Achmad, Balza & D1RB.4 \\
\hline Adi, Prajanto Wahyu & D1RC.13 \\
\hline Adiprawita, Widyawardana & D1RC.16 \\
\hline Adyanti, Deasy & D2RA.14 \\
\hline Afandi, AN & D1RE.7 \\
\hline Affandi, Achmad & D2RA.15 \\
\hline Agus, Fahrul & D1RB.9 \\
\hline Agustika, Dyah & D1RB.10 \\
\hline Ahmad, Mashhood & D2RC.6 \\
\hline Aini, Nur & D1RB.9 \\
\hline \multirow[t]{2}{*}{ Ainul Yaqin, Muhammad } & D1RD.1 \\
\hline & D1RD.9 \\
\hline Akbar, Saiful & D2RD.10 \\
\hline Akef, Fatiha & D2RB.1 \\
\hline Al Amin, Imam & D1RC.10 \\
\hline Al-Ali, Zahraa & D2RB.13 \\
\hline Al-Jumaily, Adel & D1RB.2 \\
\hline Alam, Syah & D2RC.4 \\
\hline Alameka, Faza & D2RC.11 \\
\hline Alfiadi, Heldi & D1RE.6 \\
\hline Ali Pacha, Adda & D2RA.16 \\
\hline Ali Pacha, Hana & D2RA.16 \\
\hline Amany, Alia Shaliha & D2RC.8 \\
\hline Anam, Khairul & D1RB.2 \\
\hline \multirow[t]{2}{*}{ Andromeda, Trias } & D1RA.7 \\
\hline & D1RA.10 \\
\hline Andy, Syaiful & D2RC. 2 \\
\hline
\end{tabular}

Anggrainingsih, Rini

D2RD.12 735

Anza, Fikri

Arianto, Ahmad Dwi

Arifin, Zainal

Ariyanto, Mochammad

Ariyanto, Yuri

Arymurthy, Aniati

Ashari, Ahmad

Assegaf, Badieah

Astuti, Yani

Asyhar, Ahmad

Aulia, Atqia

Azainil, Azainil

Azhari, Azhari

Bahaweres, Rizal

Boedinoegroho, Hanny

Budaya, Gilang

Budiman, Edy

Burhanuddin, Auliya

Candra, Sevenpri

Chu, Shiao

Dahono, Pekik

Das, Arijit

Dasuki, Massolihen

Delimayanti, Mera Kartika

Dewi, Tresna

Diana, Diana

Djamal, Esmeralda

Dwi Ramadhon, Bagus
D2RD. 8713

D2RA.15 498

D1RB.9 138

D2RB.3 525

D2RB.9 556

D1RA.1 9

D1RA.15 88

D1RD.4 277

D2RD.15 751

D1RC.13 239

D2RA.14 492

D1RA.3 21

D1RB.9 138

D1RD.7 294

D1RA.3 21

D1RA.14 82

D1RE.15 418

D1RC.14 244

D1RB.5 120

D2RC.11 647

D2RB. 6540

D1RA. $8 \quad 44$

D2RA.3 441

D2RB.12 573

D1RB.8 134

D1RE. 8383

D1RA.16 94

D1RA.4 25

D1RB.13 160

D1RA.5 30

D2RB.5 535

D1RA.8 44

D1RB. 198

D1RC.15 250

D1RE.10 395 


\begin{tabular}{|c|c|c|c|c|c|}
\hline & & & Hapsara, Manik & D1RD.10 & 313 \\
\hline Effendi, Yutika & D2RD. 2 & 678 & Harb, Hani & D2RC.14 & 664 \\
\hline \multirow[t]{2}{*}{ El Ouarrak, Hala } & D2RB.1 & 513 & Hariadi, Mochamad & D1RD.6 & 289 \\
\hline & D2RB.7 & 544 & Harijanto, Budi & D1RA.1 & 9 \\
\hline \multirow[t]{2}{*}{ Esmaeili, Seyed Ebrahim } & D2RB.13 & 579 & Harjoko, Agus & D1RD.7 & 294 \\
\hline & & & Haryadi, Bagus & D1RB.8 & 134 \\
\hline Fahanani, Agwin Fahmi & D1RB.6 & 126 & Haryaka, Usfandi & D2RC.11 & 647 \\
\hline Fahrizal, Rian & D2RC.12 & 653 & Has, Zulfatman & D2RB.2 & 519 \\
\hline Faiq, Mohammad & D1RB.4 & 114 & Hasibuan, Zainal & D1RA.12 & 68 \\
\hline Fajarianto, Otto & D1RC.14 & 244 & Hastono, Tri & D1RE.16 & 424 \\
\hline \multirow[t]{2}{*}{ Fanany, Ivan } & D1RA.15 & 88 & Hatta, Heliza & D1RB.9 & 138 \\
\hline & D1RC.11 & 233 & Hendradjaya, Bayu & D2RD.5 & 696 \\
\hline Fatoni, Fendi & D1RE.5 & 367 & Hidayat, Risanuri & D1RC.9 & 223 \\
\hline \multirow[t]{2}{*}{ Fauzan, Abd. Charis } & D1RD.1 & 261 & Hidayati, Anita & D2RA.7 & 461 \\
\hline & D1RD.9 & 306 & Hudayani, Nurul & D1RE.14 & 414 \\
\hline Fauziah, Dini & D1RE.6 & 371 & Husein, Inne & D2RD.10 & 723 \\
\hline Francis, Tina & D2RA.4 & 446 & Husni, Nyayu & D1RA.5 & 30 \\
\hline Fujita, Goro & D1RE.7 & 377 & Husni, Nyayu & D2RB.14 & 584 \\
\hline \multirow{5}{*}{$\begin{array}{l}\text { Gaballah, W. } \\
\text { Gunawan, Fergyanto }\end{array}$} & D2RC.14 & 664 & Ichsan, Mochammad Hannats & D1RA.2 & 15 \\
\hline & D1RA.8 & 44 & Hanafi & & 25 \\
\hline & D1RD.10 & 313 & Idris, Mond. Y azid & DIRA.4 & 25 \\
\hline & D2RA.3 & 441 & Idris, Nik Rumzi Nik & INV.1 & 1 \\
\hline & D2RB.12 & 573 & Illeperuma, Gayan & D1RC.7 & 212 \\
\hline \multirow{2}{*}{ Gunawan, Hendra } & D2RD. 6 & 702 & Ilyas, Ridwan & D1RC.15 & 250 \\
\hline & & 102 & Inoerawan, Abraham & D2RA.9 & 472 \\
\hline Habibullah, Nik & D1RA.4 & 25 & Irawan, Dedi & D2RB. 10 & 562 \\
\hline \multirow{2}{*}{ Hadary, Ferry } & D1RE.12 & 405 & Isabella, Isabella & D1RD.2 & 267 \\
\hline & D1RC 10 & 228 & Ismail, Mohammad & D1RD.5 & 283 \\
\hline $\begin{array}{l}\text { Hadikurniawati, Wiwien } \\
\text { Hadj Said, Naima }\end{array}$ & D2RA.16 & $\begin{array}{l}220 \\
503\end{array}$ & Ismail, Rifky & D2RB.3 & 525 \\
\hline Haeruddin, Haeruddin & D1RB.5 & 120 & \multirow[b]{2}{*}{ Istardi, Didi } & \multirow{2}{*}{$\begin{array}{l}\text { D2RB.9 } \\
\text { D1RE.1 }\end{array}$} & \multirow{2}{*}{$\begin{array}{l}556 \\
353\end{array}$} \\
\hline Hafiyusholeh, Moh. & D2RA.14 & 492 & & & \\
\hline \multirow{4}{*}{$\begin{array}{l}\text { Haider, Syed Turab } \\
\text { Hairah, Ummul } \\
\text { Hakiem, Nashrul }\end{array}$} & D2RC.6 & 619 & \multirow{5}{*}{$\begin{array}{l}\text { Jefiza, Adlian } \\
\text { Jihane, Chaimaa } \\
\text { Johan, Herni } \\
\text { Juyuspan, Fityan }\end{array}$} & \multirow{5}{*}{$\begin{array}{l}\text { D1RE.15 } \\
\text { D2RB.7 } \\
\text { D1RB.5 } \\
\text { D2RA.7 }\end{array}$} & \multirow{2}{*}{418} \\
\hline & D1RB.5 & 120 & & & \\
\hline & D1RA.3 & 21 & & & 544 \\
\hline & D1RA.14 & 82 & & & 120 \\
\hline Hammad, Jehad & D1RA.13 & 76 & & & 461 \\
\hline Hananto, Mursid & D1RD.4 & 277 & \multirow{2}{*}{ Kagawa, Takuya } & \multirow{2}{*}{ D2RD.9 } & \\
\hline \multirow{2}{*}{$\begin{array}{l}\text { Handayani, Ade } \\
\text { Handayani, Ade }\end{array}$} & D1RA.5 & 30 & & & 719 \\
\hline & D2RB.14 & 584 & Kambau, Ridwan & D1RA.12 & 68 \\
\hline \multirow[t]{2}{*}{ Handoko, Fourry } & D1RC.1 & 181 & Karna, Nyoman & D2RA.5 & 451 \\
\hline & D1RE.13 & 409 & \multirow{2}{*}{$\begin{array}{l}\text { Karyawati, A. A. I. N. Eka } \\
\text { Kassim, Siti Rafidah }\end{array}$} & D1RD.7 & 294 \\
\hline Hanindhito, Bagus & D2RC. 2 & 600 & & D1RB.14 & 165 \\
\hline
\end{tabular}




\begin{tabular}{|c|c|c|c|c|c|}
\hline Kausar, Engkoos & D1RE.10 & 395 & Mustofa, Khabib & D1RD.4 & 277 \\
\hline Kemala Putri, Vinnia & D1RC.11 & 233 & Muttakin, Imamul & D2RC.8 & 631 \\
\hline \multirow[t]{2}{*}{ Khairani, Dewi } & D1RA.3 & 21 & & & \\
\hline & D1RA.14 & 82 & Na`am, Jufriadif & D1RA.11 & 61 \\
\hline Khairudin, Moh & D1RC.3 & 192 & Naiwala P., Chandrasiri & D2RD.9 & 719 \\
\hline \multirow[t]{2}{*}{ Khayam, Umar } & D1RE.5 & 367 & Napitupulu, Darmawan & D2RD.4 & 690 \\
\hline & D1RE.11 & 399 & Nari, Mochamad Irwan & D1RC.16 & 256 \\
\hline Khidzir, Nik Zulkarnaen & D1RD.5 & 283 & Nasution, Feldiansyah & D2RD.1 & 672 \\
\hline Khodra, Masayu Leylia & D1RC.5 & 202 & Negara, Ridha & D2RC.13 & 658 \\
\hline Khoirudin, Nachrowi & D2RD.12 & 735 & Ngah, Razali & D2RC.5 & 614 \\
\hline Kosasi, Sandy & D1RD.11 & 319 & Ningsih, Yuli Kurnia & D2RC.4 & 610 \\
\hline Kurniawan, Arief & D1RB.15 & 170 & Notosudjono, Didik & D1RE.10 & 395 \\
\hline Kurniawan, Fachrul & D1RD.6 & 289 & Novianty, Cindy & D2RA.7 & 461 \\
\hline \multirow[t]{2}{*}{ Kurniawan, Novianto } & D1RD.14 & 330 & Novitasari, Dian & D2RA.14 & 492 \\
\hline & D1RD.17 & 347 & Nugroho, Hanung & INV.2 & 8 \\
\hline Kurniawan, Wijaya & D1RA.2 & 15 & & D1RC.9 & 223 \\
\hline \multirow[t]{2}{*}{ Kusumadewi, Sri } & D2RA.1 & 430 & Nurhamidah, Neneng & D1RC.15 & 250 \\
\hline & & & Nurlaili, Afina & D1RD.15 & 336 \\
\hline Langsari, Kholed & D2RA. 8 & 466 & Nurmaini, Siti & D1RA.5 & 30 \\
\hline Lastomo, Dwi & D1RE.9 & 389 & & D2RB.14 & 584 \\
\hline Lestari, Sri & D1RB.12 & 156 & Nuryanto, Nuryanto & D2RB.6 & 540 \\
\hline Lin, Gen & D1RB.8 & 134 & & & \\
\hline Lodaya, Poppi & D1RB.1 & 98 & Octaviani, Vika & D1RB. 15 & 170 \\
\hline Loo, Hui Ru & D1RA.10 & 55 & Oktarina, Yurni & D2RB.5 & 535 \\
\hline \multirow[t]{2}{*}{ Lubab, Ahmad } & D2RA.14 & 492 & Osman, azlan & D2RC.12 & 653 \\
\hline & & & Osman, Salyani & D2RA.2 & 436 \\
\hline Maarif, Muhammad & D2RA.12 & 482 & & & \\
\hline Mardi Suryanto, Tri & D1RD.3 & 271 & Pambudi, Kharisma & D2RB.3 & 525 \\
\hline Marjudi, Suziyanti & D2RA.2 & 436 & Partadiredja, Ginus & D1RB.4 & 114 \\
\hline \multirow[t]{2}{*}{ Marsono, Muhammad Nadzir } & D1RA.7 & 39 & Pertiwi, Annas & D2RD.14 & 746 \\
\hline & D1RA.10 & 55 & Phuc Khai, Nguyen & D1RE.7 & 377 \\
\hline \multirow[t]{2}{*}{ Marwanto, Arief } & D2RC.10 & 642 & Pola Risma, Risma & D2RB.5 & 535 \\
\hline & D2RC.14 & 664 & Prabowo, M. Roynaldi & D1RE.14 & 414 \\
\hline Matulatan, Tekad & D2RA.10 & 477 & Prabowo, Martin & D1RA.9 & 49 \\
\hline Maulidevi, Nur Ulfa & D2RA.5 & 451 & Prakoso, Teguh & D1RB.16 & 176 \\
\hline \multirow[t]{2}{*}{ Mestari, Mohammed } & D2RB.1 & 513 & & D2RC. 5 & 614 \\
\hline & D2RB.7 & 544 & Pramunanto, Eko & D1RE.15 & 418 \\
\hline Mohd Rahim, Mohd Shafry & D1RC.14 & 244 & Pranowo, Pranowo & D1RE.16 & 424 \\
\hline Mohd Saad, Norhashimah & D1RC.4 & 196 & Praptodiyono, Supriyanto & D2RC.12 & 653 \\
\hline Monemi, Alireza & D1RA.10 & 55 & Pratama, M. Ardhika & D1RA.9 & 49 \\
\hline Muhimmah, Izzati & D2RA.1 & 430 & Pratama, Rizki & D1RE.12 & 405 \\
\hline Munir, Munir & D1RD.6 & 289 & Prihatini, Rahayu Suci & D1RC. 8 & 218 \\
\hline Muqorobin, Anwar & D1RE.8 & 383 & Priyandoko, Gigih & D1RC.14 & 244 \\
\hline
\end{tabular}




\begin{tabular}{|c|c|c|c|c|c|}
\hline Pujotomo, Isworo & D1RE. 2 & 358 & Sanou, Ibrahim & D2RB.1 & 513 \\
\hline Purnomo, Mauridhi & D1RE.15 & 418 & Santoso, Albertus & D1RE.16 & 424 \\
\hline Purnomo WP, Yohanes Sigit & D2RA.13 & 487 & Saputra, Wardianto Eko & D2RD.15 & 751 \\
\hline Purwadi, Agus & D1RE.8 & 383 & Sari, Christy & D1RC.2 & 187 \\
\hline Purwiyanti, Sri & D1RE.14 & 414 & Sari, Widya & D1RB.14 & 165 \\
\hline Putri, Hardika & D2RC.13 & 658 & Sarno, Riyanarto & D1RD.1 & 261 \\
\hline \multirow[t]{2}{*}{ Putro Sarwo Setyohadi, Dwi } & D1RA.6 & 35 & & D1RD.9 & 306 \\
\hline & & & & D1RD.15 & 336 \\
\hline \multirow[t]{2}{*}{ Qonita, Adiba } & D2RD.14 & 746 & & D1RD.16 & 341 \\
\hline & & & & D2RA.8 & 466 \\
\hline \multirow[t]{2}{*}{ Rachik, Mostafa } & D2RB. 1 & 513 & & D2RD.2 & 678 \\
\hline & D2RB.7 & 544 & & D2RD.3 & 684 \\
\hline Rachman, Anung & D1RC.9 & 223 & Satria, Haikal & D1RB.11 & 150 \\
\hline Rachmawanto, Eko & D1RC.2 & 187 & Satria, Muhammad & D2RC.10 & 642 \\
\hline Rachmawati, Ema & D1RC.5 & 202 & Sembiring, Jaka & D1RD.14 & 330 \\
\hline Rahardjo, Budi & D2RC. 2 & 600 & & D1RD.17 & 347 \\
\hline Rahman, Arif & D1RC.6 & 208 & Sensuse, Dana & D2RD.4 & 690 \\
\hline Rahmawati, Dewi & D1RD.16 & 341 & & D2RD.8 & 713 \\
\hline \multirow{3}{*}{$\begin{array}{l}\text { Raja Lope Ahmad, Raja Mohd } \\
\text { Tariqi } \\
\text { Ramadhan, Arief }\end{array}$} & D1RD.12 & 324 & Setiadi, De Rosal Ignatius Moses & D1RC.2 & 187 \\
\hline & 8 DPD 8 & & Setiadi, Haryono & D2RD.12 & 735 \\
\hline & D2KD.8 & 113 & Setiadi, Herlambang & D1RE.9 & 389 \\
\hline Ramadiani, Ramadiani & D1RB.9 & 138 & Setianingrum, Anif & D1RC. 8 & 218 \\
\hline Ramli, Kalamullah & D1RB.3 & 110 & Setiawan, Agung & D1RC.16 & 256 \\
\hline Rantelinggi, Parma & D2RC.1 & 596 & Setiawan, Joga & D2RB.9 & 556 \\
\hline Resha, Muhammad & D2RA.10 & 477 & Setyawan, Arinto & D1RE.14 & 414 \\
\hline Retnowardhani, Astari & D2RA.9 & 472 & Setyohadi, Djoko & D1RD.3 & 271 \\
\hline Riadi, Imam & D2RA.6 & 456 & & D2RA.13 & 487 \\
\hline Riandini, Riandini & D1RB.13 & 160 & Sfenrianto, Sfenrianto & D2RD.7 & 708 \\
\hline Riskiawan, Hendra & D1RA.6 & 35 & Shaikh-Husin, Nasir & D1RA.7 & 39 \\
\hline \multirow[t]{2}{*}{ Riyadi, Munawar } & D1RB.16 & 176 & Shepeta, Alexander & $\mathrm{D} 2 \mathrm{RC} .3$ & 605 \\
\hline & D2RC. 5 & $\begin{array}{r}614 \\
25\end{array}$ & Shofi, Imam & D1RC.8 & 218 \\
\hline Rizaldi, Taufiq & D1RA.6 & 35 & Sirkunan, Jeevan & D1RA.7 & 39 \\
\hline Rochmanto, Raditya & DIRB.7 & $\begin{array}{l}129 \\
525\end{array}$ & Sitohang, Benhard & D2RD.10 & 723 \\
\hline \multirow{2}{*}{$\begin{array}{l}\text { Roseno, M } \\
\text { Rosyadi, Ahmad }\end{array}$} & $\begin{array}{l}\text { DLRB.5 } \\
\text { D1PD? }\end{array}$ & 535 & Soewito, Benfano & D1RA.8 & 44 \\
\hline & DIRB.2 & 104 & & D1RD.10 & 313 \\
\hline \multirow{2}{*}{ S, Muhammad } & D2RA.7 & 461 & & D2RA.3 & 441 \\
\hline & D2RA.13 & 487 & & D2RB. 12 & 573 \\
\hline Sabatini, Gabriella & D1RA 16 & 94 & Sofhan, Rian & D2RC. 12 & 653 \\
\hline Saha, Diganta & D2RA 17 & 507 & Sonnadara, Upul & D1RC.7 & 212 \\
\hline \multirow{2}{*}{$\begin{array}{l}\text { Saleem, Muhammad Mubasher } \\
\text { Samra, Ahmed }\end{array}$} & $\begin{array}{l}\text { D2RA.17 } \\
\text { D2RC.6 }\end{array}$ & 619 & Sorokin, Alexander & D2RC. 3 & 605 \\
\hline & D2RC. 14 & 664 & Stiawan, Deris & D1RA.4 & 25 \\
\hline \multirow{2}{*}{ Sanmorino, Ahmad } & D1RD 2 & 267 & Subhiyakto, Egia & D1RC.13 & 239 \\
\hline & & 201 & Subrata, Aan & D1RD.14 & 330 \\
\hline
\end{tabular}




\begin{tabular}{|c|c|c|c|c|c|}
\hline Subroto, Imam Much Ibnu & D2RD.15 & 751 & Wan Abdul Ghani, Wan Safra & D1RD.5 & 283 \\
\hline Sucahyo, Yudho & D2RD.4 & 690 & Diyana & & \\
\hline \multirow[t]{2}{*}{ Suhardi, Suhardi } & D1RD.14 & 330 & Wan Ismail, Wan Basri & D1RD.12 & 324 \\
\hline & D1RD.17 & 347 & Wanandi, Krisantus & D2RB. 12 & 573 \\
\hline Suharyanto, Cosmas & D1RA.8 & 44 & Wattimena, Gysberth & D2RC.3 & 605 \\
\hline Suhendi, Dede & D1RE.10 & 395 & Watulingas, Jefferson & D2RC.11 & 647 \\
\hline Sujanarko, Bambang & D1RB.2 & 104 & Wibawa, Aji & D1RD.6 & 289 \\
\hline Sukur, Muji & D1RC.10 & 228 & Wibowo, Moh & D1RC.6 & 208 \\
\hline Sulistiyanti, Sri & D1RE.14 & $\begin{array}{l}228 \\
414\end{array}$ & Wibowo, Nur & D1RD.3 & 271 \\
\hline Sulistyorini, Yunis & D1RE.7 & 377 & Widianto, Widianto & D1RE.4 & 362 \\
\hline Sungkono, Kelly & D2RD.3 & 684 & Widiyaningtyas, Triyanna & D1RA.9 & 49 \\
\hline Suprapto, Yoyon & D1RB. 15 & 170 & & D2RD.14 & 746 \\
\hline \multirow[t]{2}{*}{ Supriana, Iping } & D1RC.5 & 202 & Widiyanto, Andi & D2RB.6 & 540 \\
\hline & D2RA.5 & 451 & Widodo, Nuryono & D2RB.4 & 531 \\
\hline Surjati, Indra & D2RC.4 & 610 & Widyantoro, Dwi & D2RD.5 & 696 \\
\hline Suroso, Jarot S & D2RA.9 & 472 & Widyarto, Setyawan & D1RB.14 & 165 \\
\hline Suryasa, Nyoman & D1RC.14 & 244 & & D1RC.14 & 244 \\
\hline Suryawan, Fajar & D2RB. 15 & 590 & & D1RD.12 & 324 \\
\hline \multirow{3}{*}{$\begin{array}{l}\text { Suryono, Suryono } \\
\text { Susiki Nugroho, Supeno Mardi }\end{array}$} & D2RB.11 & 567 & & D2RA.2 & 436 \\
\hline & D1RD.6 & 289 & Wijaya, Oky & D1RA. 8 & 44 \\
\hline & D2RA. 15 & 498 & Winarko, Edi & D1RC.6 & 208 \\
\hline Sutikno, Tole & INV.1 & 1 & & D1RD.7 & 294 \\
\hline Suwarno, Dr & D1RE.6 & 371 & Winarno, Edy & D1RC.10 & 228 \\
\hline Suyadi, Suyadi & D1RE.3 & & Winiarti, Sri & D2RA.1 & 430 \\
\hline Syaifudin, Yan & D1RA.1 & 9 & Wiryadinata, Romi & D2RC.8 & 631 \\
\hline Syed-Yusof, Sharifah & D2RC.10 & 642 & Wu, Hsien-Tsai & D1RB.8 & 134 \\
\hline Tahyudin, Imam & D2RD.11 & 729 & Yacoub, Redi & D1RE.12 & 405 \\
\hline Talib, Shukor & D1RB.11 & 150 & Yang, Chieh & D1RB.8 & 134 \\
\hline Tan, Tse Guan & D1RD.5 & 283 & Yani, Irsyadi & D1RA.5 & 30 \\
\hline Taufik, Mohammad & D1RE.9 & 389 & Yani, Irsyadi & D2RB.14 & 584 \\
\hline Tjahjadi, Hendrana & D1RB.3 & 110 & Yel, Mesra & D2RD.7 & 708 \\
\hline \multirow[t]{2}{*}{ Tjahjadi, Martinus } & D1RC.1 & 181 & Yohanes, Banu & D2RC.7 & 626 \\
\hline & D1RE.13 & 409 & Yovita, Leanna & D2RC.13 & 658 \\
\hline Tutkun, Nedim & D1RE.7 & 377 & Yuliani, I Dewa Ayu Eka & D1RD.11 & 319 \\
\hline & & & Yuliansyah, Herman & D2RA.1 & 430 \\
\hline Ungkawa, Uung & D2RD. 5 & 696 & Yulita, Intan & D1RA.15 & 88 \\
\hline Utama, Yoga & D2RB.8 & 550 & Yunianta, Arda & D1RD.8 & 300 \\
\hline Utami, Ema & D2RD.13 & 739 & Yusa, Mochammad & D2RD.13 & 739 \\
\hline 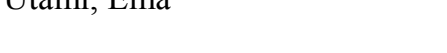 & DLND.1 & 109 & Yusuf, Yushan & D1RE.11 & 399 \\
\hline Vedyanto, Vedyanto & D1RD.11 & 319 & Zaini, Ahmad & D1RB.15 & 170 \\
\hline Waluyo, Catur & D2RC. 9 & 637 & Zawawi, Khoirunnisya & D1RA.14 & 82 \\
\hline
\end{tabular}





\section{http://eecsi_org/2017}

\section{EECSI 2017 \\ CONFERENCE}

Yogyakarta, Indonesia

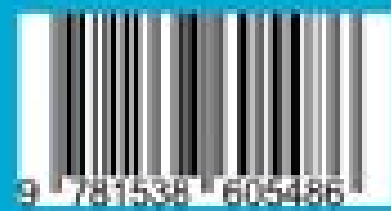

